\title{
Neoichnology of two spirobolid millipedes: improving the understanding of the burrows of soil detritivores
}

\author{
Jared J. Bowen and Daniel I. Hembree
}

\begin{abstract}
Burrowing detritivores such as millipedes play an important role in soil formation. In order to improve the recognition of millipede burrows and, therefore, the presence of millipede macrodetritivores in the fossil record, this study describes the burrowing behavior and resulting burrow morphologies of two species of spirobolid millipedes: Narceus americanus and Floridobolus penneri. Millipedes were placed in terraria filled with sediment of varied compositions and controlled moisture contents for 7-94 days. Open burrows were cast with plaster, excavated, and described both qualitatively and quantitatively. Both $N$. americanus and $F$. penneri produced vertical, subvertical, heli$\mathrm{cal}$, and O-shaped burrows. Floridobolus penneri also produced J-shaped burrows. Burrow morphologies of both species were found to be similar and unaffected by sediment properties using the Bray Curtis similarity and Spearman's rank correlation tests, respectively. Burrows of two other millipede species of the Order Spirostreptida, Orthoporus ornatus, and Archispirostreptus gigas, produced in a previous study were also compared to the spirobolid burrows. While burrows of $O$. ornatus were similar, burrows produced by $A$. gigas, the largest of the four species, were the least similar even after removing size-dependent burrow properties from the analysis. Differences and similarities in burrow morphology were instead attributed to the behavioral function of the burrow: refuge or feeding. Despite some differences, all millipede burrow shafts and tunnels had a mean width-to-height ratio of 1.0-1.14. These results show that juliform millipedes produce burrows with unique morphologies that may be used to differentiate their burrows from those produced by other soil organisms.
\end{abstract}

Jared J. Bowen. Department of Geological Sciences, Ohio University, 316 Clippinger Laboratories, Athens, Ohio 45701, USA. jb836611@ohio.edu

Daniel I. Hembree. Department of Geological Sciences, Ohio University, 316 Clippinger Laboratories, Athens, Ohio 45701, USA. hembree@ohio.edu

Keywords: ichnofossils; trace fossils; bioturbation; continental; arthropod; paleosol

Bowen, Jared J. and Hembree, Daniel I. 2014. Neoichnology of two spirobolid millipedes: improving the understanding of the burrows of soil detritivores. Palaeontologia Electronica Vol. 17, Issue 1;18A; 48p; palaeo-electronica.org/content/2014/709-neoichnology-of-spirobolids 


\section{INTRODUCTION}

Trace fossils include preserved tracks, trails, burrows, and borings that result from the life activities of an organism within or on a medium (Bromley, 1996). These structures represent the direct response of an organism to its environment (Bromley, 1996). As environmental conditions (sediment composition, moisture, temperature, etc.) change, the behavior of the organism and thus the trace may also change (Davis et al., 2007; Hasiotis, 2007; Genise et al., 2009; Hembree, 2009; Hamer and Sheldon, 2010; Dashtgard, 2011; Hembree et al., 2012, Smilek and Hembree, 2012, Getty et al., 2013). As such, an understanding of trace fossils and their potential tracemakers is invaluable to paleoenvironmental and paleoecological reconstructions. Understanding the significance of trace fossils comes from experimental neoichnology. These studies involve the observation of extant organisms in the field or controlled laboratory settings while they are interacting with a medium. Observations of the organism's behaviors and reactions to changing conditions, as well as detailed documentation of the traces produced, allow these structures to serve as analogs for trace fossils.

With over 12,000 species described, the class Diplopoda (millipedes) is among the most diverse groups of extant terrestrial organisms (Sierwald and Bond, 2007). Although these animals are extremely common, their trace-making abilities are poorly understood (Hembree, 2009). It is well documented that arthropods, specifically diplopods, were among the first macrofauna to colonize land (Almond, 1985; Rolfe, 1985; Jeram et al., 1990; Pisani et al., 2004; Ward et al., 2006; Schaefer et al., 2010). Fossil millipedes have been found in deposits as old as the Silurian (Wilson and Anderson, 2004). Relatively few fossil millipedes have been described, however, likely due to their terrestrial habitats as well as their tendency to consume their molts (Shear and Edgecombe, 2010). This bias is very evident in the Permian, which lacks any well-described fossils (Shear and Edgecombe, 2010). The primary exceptions to this taphonomic bias occur in the Mississippian and Pennsylvanian which have yielded some exceptionally well-preserved fossils (Hoffman, 1963; Wilson, 2005a, 2005b, 2006a).

Fossil trackways attributed to millipedes occur as early as the Late Ordovician (Johnson et al., 1994), and coprolites attributed to millipedes have been described from the Early Devonian (Edwards et al., 2012). A fully fused, ring-form body similar to that of extant burrowing members of the Juliformia appears in the Early Devonian (Wilson, 2006b; Cong et al., 2009). Undisputed millipede burrows of that age, however, have yet to be described, although burrows in the Ordovician Juniata Formation have been suggested to have been produced by millipedes (Retallack and Feakes, 1987; Retallack, 2001a). Given the early occurrence of burrowing adaptations, fossil burrows may be an effective proxy for the presence of millipedes if they could be distinguished from those of other burrowing animals. With the higher preservation potential of trace fossils, the recognition of fossil millipede burrows could also assist in refining the date for the colonization of land.

Extant millipedes are globally widespread, but individual species are only able to tolerate small environmental changes (Golovatch and Kime, 2009). Many studies of extant millipedes have illustrated their sensitivity to precise levels of light, temperature, moisture, food resources, calcium, nitrogen, and specific landscapes, all factors that would greatly assist in paleoenvironmental reconstructions (Crozier, 1924; O'Neill, 1967, 1968a, 1968b, 1969; Toye, 1966; Banerjee, 1967; Davis, 1978; Moeed and Meads, 1985; Crawford, 1992; Bailey and Kovaliski, 1993; Dangerfield and Chipfunde, 1995; Cárcamo et al., 2000; Greyling et al., 2001; Kalisz and Powell, 2003; Tuf et al., 2006; Farfan, 2008; Stašiov, 2009; Stašiov et al., 2012; Fujimaki et al., 2010; Kime and Golovatch, 2000; Galanes and Thomlinson, 2011).

The purpose of this paper is to characterize biogenic structures produced by two extant species of spirobolidan millipedes (Myriapoda: Diplopoda: Juliformia), Narceus americanus (Family: Spirobolidae), and Floridobolus penneri (Family: Floridobolidae), under controlled laboratory conditions. Surface and subsurface biogenic structures produced by $F$. penneri and $N$. americanus are thoroughly described along with the associated burrowing techniques and behaviors. Sediment composition, moisture, available space, and time available were varied to determine the effect of these variables on burrow morphology. By describing the biogenic structures produced by such common extant soil detritivores as millipedes under varying environmental conditions, trace fossils with similar morphologies may be used to aid in the interpretation of paleoenvironmental, paleoecologic, and paleoclimatic conditions of continental deposits. 


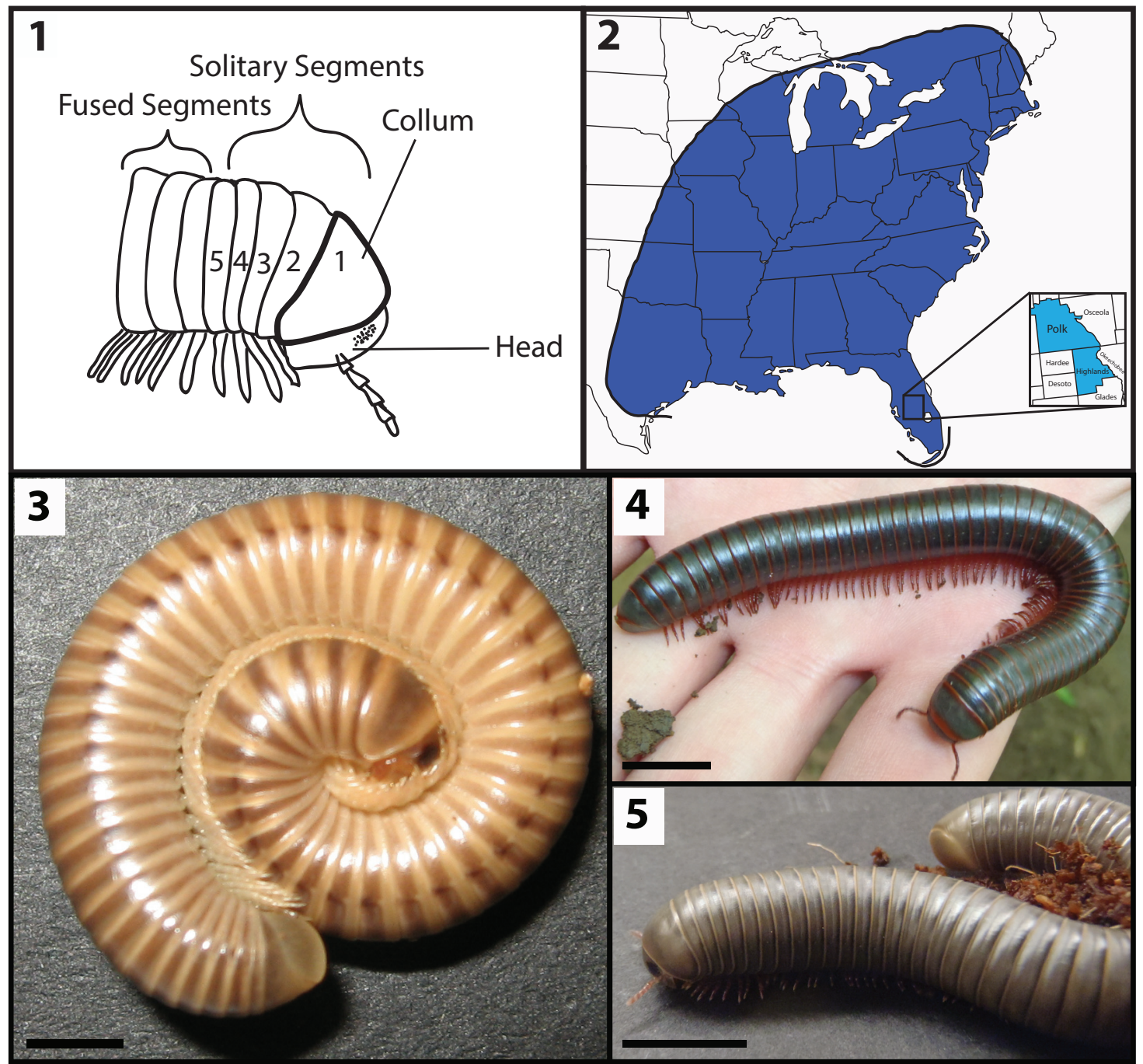

FIGURE 1. Millipede morphology and distribution. 1. Diagram of Narceus americanus showing the head, first five segments, and three diplosegments. 2. Distribution map of $N$. americanus after Shelley et al. (2006). Inset map shows the distribution of Floridobolus penneri in central Florida. 3. Coiled N. americanus individual used in this study. 4. Narceus americanus from the Smoky Mountains, Tennessee. 5. A F. penneri individual used in this study. Scale bar equals 1 $\mathrm{cm}$.

\section{MILLIPEDE ECOLOGY AND BEHAVIOR}

The Class Diplopoda is divided into 16 extant orders and 8 extinct orders distributed over every continent except for Antarctica (Golovatch and Kime, 2009). Millipedes are present in most terrestrial habitats living primarily at or near the leaf litter/ soil interface (Sierwald and Bond, 2007). The typical environments for millipedes are temperate to tropical, humid deciduous forests; however, they can also thrive at high altitudes, in caves, and in deserts (Golovatch and Kime, 2009). Millipedes are characterized by an elongate body form consisting of multiple fused segments (diplosegments), with two pairs of legs and two pairs of tracheae on all but the head and first several segments (Figure
1.1) (Sierwald and Bond, 2007). Most millipedes have a calcite-impregnated, chitinous exoskeleton that provides physical defense and protection against desiccation (O'Neill, 1969; Thorez et al., 1992; Eisner et al., 1998; Shear and Edgecombe, 2010). As a result, many millipedes have a preference toward calcium-rich environments (Kime and Golovatch, 2000). The diplosegment condition increases pushing force capability while the calcite reinforced cuticle protects the animal as it pushes itself into sediment using the collum as the primary force point (Manton, 1953, 1958, 1961; Borrell, 2004).

Among millipedes, members of the orders Polydesmida, Spirostreptida, Spirobolida, and Jul- 
ida are best adapted to burrowing and feeding in soils (Enghoff et al., 1993; Cong et al., 2009). Three specific morphotypes of burrowing millipedes have been described: (1) juloid, or rammingtype body forms chiefly consisting of members of the Superorder Juliformia, that use a compressive burrowing technique; (2) polydesmoid, or wedgetype body forms, that force their way into cracks or crevices and then lift their bodies to create wider openings; and (3) platydesmoid, or borer-type body forms, with flat backs and tapered anterior ends that are pushed into the sediment (Kime and Golovatch, 2000). Millipedes are known to increase their energy output in response to stressed conditions and typically burrow to avoid desiccation (O'Neill, 1967, 1968a; Dangerfield and Chipfunde, 1995; Villani et al., 1999).

Millipedes are primary detritivores and, therefore, play an important role in the initial breakdown of plant material (Ausmus, 1977; Crawford, 1992). In temperate forests they are estimated to consume up to $15 \%$ of annual leaf fall and up to $36 \%$ of the annual litter in coniferous forests (Golovatch and Kime, 2009). Decomposition of litter by millipedes can increase the overall rate of nitrogen mineralization and influence the microbial activity of soils (Cárcamo et al., 2000). It is clear that millipedes presently play an important part in the formation of soils in many ecosystems and may have played an even larger role in the past since the evidence of terrestrial millipedes predates the appearance of other macrodetritivores by at least 27 million years (Crawford, 1992).

Both Narceus americanus and Floridobolus penneri are of the juloid morphotype and are known to burrow, but live in different habitats. Narceus americanus is a large, North American millipede that can reach up to $10 \mathrm{~cm}$ in length as an adult (Figure 1.2-4) (Shelley et al., 2006). The species occurs in the eastern half of the United States and portions of southeastern Canada at all elevations primarily on temperate to subtropical forest floors (O'Neill, 1968a, 1968b; Ausmus, 1977; Shelley et al., 2006). Narceus americanus has a relatively high tolerance for dry conditions which likely accounts for its widespread distribution (O'Neill, 1969; Shelley et al., 2006; Walker et al., 2009). Narceus americanus is primarily active at night in the spring; during the daylight hours and in winter $N$. americanus burrows into the soil to avoid desiccating conditions and cold temperatures, respectively (Walker et al., 2009). Floridobolus penneri, also known as the Florida scrub millipede, is a large, North American millipede that reaches an adult length of $9 \mathrm{~cm}$ (Figure 1.2, 1.5). The species is restricted to xeric, infertile, sandy scrub in south central Florida (Carrel and Britt, 2009). Little has been published regarding the ecology of $F$. penneri, but it is known to spend a majority of the time in its burrows and is mostly surface active in the mid-summer (Carrel and Britt, 2009). Its feeding habits have not been well-studied and are still not well known (Sattman, 2006; Carrel and Britt, 2009).

\section{MATERIALS AND METHODS}

A total of 19 individuals of Narceus americanus and 21 individuals of Floridobolus penneri were acquired from a commercial source. The mean length and width of the $N$. americanus individuals were $49 \mathrm{~mm}(27-75 \mathrm{~mm}, \mathrm{SD}=15.7)$ and 4.4 $\mathrm{mm}$ (2.5-7.0 mm, SD=1.48), respectively. The mean length and width of the $F$. penneri individuals were $68 \mathrm{~mm}(63-74 \mathrm{~mm}, \mathrm{SD}=3.66)$ and $7 \mathrm{~mm}(6-9$ $\mathrm{mm}, \mathrm{SD}=0.92$ ), respectively. All millipedes were given at least fourteen days to acclimate to the laboratory conditions.

Experiments were designed to address the hypotheses that: 1) Narceus americanus and Floridobolus penneri each produce distinct burrow morphologies; 2) the burrowing behaviors and burrows of $N$. americanus and $F$. penneri vary significantly with changes in sediment composition and moisture content; and 3) burrows produced by $N$. americanus and $F$. penneri are distinct from those produced by species from other orders of millipedes.

Twenty-three experimental trials were set up using either groups of four or five individuals or a single individual of both species (Table 1). Experiments utilized single and multiple individuals to determine if the millipedes known aggregation behavior affected burrow morphology in the form of communal burrows. Both 30 gallon (114 L) ( $91 \mathrm{~L} x$ $45 \mathrm{~W} \times 33 \mathrm{H} \mathrm{cm})$ and 65 gallon $(246 \mathrm{~L})(91 \mathrm{~L} \times 45 \mathrm{~W} \times$ $62 \mathrm{H} \mathrm{cm}$ ) terraria were used in the experiments to evaluate the effect of space limitation on the behavior of the millipedes. Each terrarium was filled with $16-20 \mathrm{~cm}$ and $39-50 \mathrm{~cm}$ of sediment, respectively. Each trial subjected the millipedes to a stress that was related to sediment composition or sediment moisture content. Sediment compositions consisted of: 1) $100 \%$ soil (clay loam) (100S), 2) $50 \%$ organic matter (coconut fiber) and $50 \%$ soil $(50 \mathrm{O} / 50 \mathrm{~S})$, 3) $50 \%$ carbonate sand (fine- to medium-grained) and $50 \%$ soil ( $50 \mathrm{Sa} / 50 \mathrm{~S})$, and 4 ) $25 \%$ carbonate sand and $75 \%$ soil ( $25 \mathrm{Sa} / 75 \mathrm{~S}$ ). The soil was passed through a $4 \mathrm{~mm}$ sieve prior to use in order to achieve better particle sorting. Initial 
TABLE 1. Experimental parameters and associated burrow architectures. Terrarium sizes are in gallons. Sediment indicates the relative percentage of $\mathrm{O}=$ organic (coconut fiber), $\mathrm{S}=$ soil (clay loam), and $\mathrm{Sa}=$ fine- to medium-grained carbonate sand. Depth is the total depth of the sediment in centimeters. \% Moisture is the mean moisture content of the sediment. Duration is split into two categories: expected time to completion of trial and the actual trial duration (in parentheses). Key to burrow architectures and modifications: $\mathrm{V}=$ vertical burrow, $\mathrm{SV}=$ subvertical burrow, $\mathrm{H}=$ helical burrow, $\mathrm{J}=\mathrm{J}$-shaped burrow, $\mathrm{O}=\mathrm{O}$-shaped burrow (T1=Type 1, T2=Type 2), c = chamber(s), b = branch(es), $\mathrm{m}=$ multiple entrances.

Experiment 1: Basic Morphology

\begin{tabular}{|c|c|c|c|c|c|c|c|c|}
\hline Species & Specimens & Terrarium & Sediment & Depth & $\%$ Moisture & Duration & Burrow \#s & $\begin{array}{c}\text { Burrow } \\
\text { Architectures }\end{array}$ \\
\hline N. americanus & 5 & 30 & $50 / 50(\mathrm{O} / \mathrm{S})$ & 20 & $37 \%$ & $7(12)$ & NA26 A-B & SV, O (T1) \\
\hline N. americanus & 5 & 65 & $50 / 50(\mathrm{O} / \mathrm{S})$ & 45 & $32 \%$ & $7(12)$ & NA23 & SV \\
\hline N. americanus & 1 & 30 & $50 / 50(\mathrm{O} / \mathrm{S})$ & 20 & $35 \%$ & $7(8)$ & No Burrow & None \\
\hline N. americanus & 1 & 30 & $50 / 50(\mathrm{O} / \mathrm{S})$ & 20 & $30 \%$ & $7(8)$ & NA24 & SVc \\
\hline N. americanus & 1 & 65 & $50 / 50(\mathrm{O} / \mathrm{S})$ & 45 & $40 \%$ & $7(7)$ & NA28 & V \\
\hline N. americanus & 5 & 30 & $50 / 50(\mathrm{O} / \mathrm{S})$ & 20 & $35 \%$ & $14(21)$ & NA25 A-B & $\mathrm{SV}, \mathrm{H}$ \\
\hline N. americanus & 5 & 65 & $50 / 50(\mathrm{O} / \mathrm{S})$ & 43 & $37 \%$ & $14(14)$ & NA21 A-F & $\mathrm{SV}, \mathrm{SVb}, \mathrm{SVc}, \mathrm{Hc}$ \\
\hline N. americanus & 1 & 30 & $50 / 50(\mathrm{O} / \mathrm{S})$ & 20 & $35 \%$ & $14(14)$ & NA1 & $\mathrm{H}$ \\
\hline N. americanus & 1 & 65 & $50 / 50(\mathrm{O} / \mathrm{S})$ & 45 & $35 \%$ & $14(19)$ & No Burrow & None \\
\hline \multicolumn{9}{|c|}{ Experiment 2: Sediment Composition } \\
\hline Species & Specimens & Tank Size & Sediment & Depth & $\%$ Moisture & Duration & Burrow \#s & $\begin{array}{c}\text { Burrow } \\
\text { Architectures }\end{array}$ \\
\hline F. penneri & 5 & 30 & $50 / 50(\mathrm{Sa} / \mathrm{S})$ & 16 & $50 \%$ & $14(94)$ & FP6 A-E & SV, OC (T1) \\
\hline F. penneri & 5 & 65 & $100(\mathrm{~S})$ & 39 & $54 \%$ & $7(8)$ & FP7 A-H & $\begin{array}{l}\text { V, Vh, SV,SVb, } \\
\text { SVcb, J }\end{array}$ \\
\hline F. penneri & 4 & 30 & $50 / 50(\mathrm{Sa} / \mathrm{S})$ & 19 & $70 \%$ & $14(54)$ & No Burrow & None \\
\hline F. penneri & 5 & 30 & $25 / 75(\mathrm{Sa} / \mathrm{S})$ & 20 & $50 \%$ & $14(14)$ & FP5 A-G & $\begin{array}{c}\text { V, SV, SVc, O (T1) } \\
\text { O (T2), Ob (T2) }\end{array}$ \\
\hline F. penneri & 1 & 30 & $50 / 50(\mathrm{Sa} / \mathrm{S})$ & 19 & $58 \%$ & $14(19)$ & FP4 A-B & $\mathrm{V}, \mathrm{H}$ \\
\hline F. penneri & 1 & 30 & $25 / 75(\mathrm{Sa} / \mathrm{S})$ & 20 & $52 \%$ & $14(41)$ & No Burrow & None \\
\hline N. americanus & 5 & 30 & $25 / 75(\mathrm{Sa} / \mathrm{S})$ & 20 & $51 \%$ & $14(23)$ & NA32 & sv \\
\hline N. americanus & 1 & 30 & $25 / 75(\mathrm{Sa} / \mathrm{S})$ & 20 & $60 \%$ & $14(14)$ & NA31 A-B & $\mathrm{O}(\mathrm{T} 2), \mathrm{Om}(\mathrm{T} 2)$ \\
\hline N. americanus & 1 & 65 & $100(\mathrm{~S})$ & 39 & $60 \%$ & $14(51)$ & NA33 A-C & $\mathrm{V}, \mathrm{H}$ \\
\hline \multicolumn{9}{|c|}{ Experiment 3: Sediment Moisture } \\
\hline Species & Specimens & Tank Size & Sediment & Depth & $\%$ Moisture & Duration & Burrow \#s & $\begin{array}{c}\text { Burrow } \\
\text { Architectures }\end{array}$ \\
\hline F. penneri & 5 & 65 & $50 / 50(\mathrm{O} / \mathrm{S})$ & 45 & $49 \%$ & $14(63)$ & FP2 A-M & $\mathrm{V}, \mathrm{SV}, \mathrm{J}, \mathrm{Jb}$ \\
\hline F. penneri & 1 & 65 & $50 / 50(\mathrm{O} / \mathrm{S})$ & 45 & $50 \%$ & $14(13)$ & FP3 A-I & V, SV \\
\hline F. penneri & 1 & 65 & $50 / 50(\mathrm{O} / \mathrm{S})$ & 45 & $50 \%$ & $14(14)$ & FP1 & $\mathrm{Hb}$ \\
\hline N. americanus & 5 & 65 & $50 / 50(\mathrm{O} / \mathrm{S})$ & 48 & $50 \%$ & $14(22)$ & NA29 A-C & $\mathrm{V}, \mathrm{H}, \mathrm{O}(\mathrm{T} 1)$ \\
\hline N. americanus & 1 & 65 & $50 / 50(\mathrm{O} / \mathrm{S})$ & 50 & $50 \%$ & $14(21)$ & NA30 A-F & V, Vh, SV, SVc \\
\hline
\end{tabular}




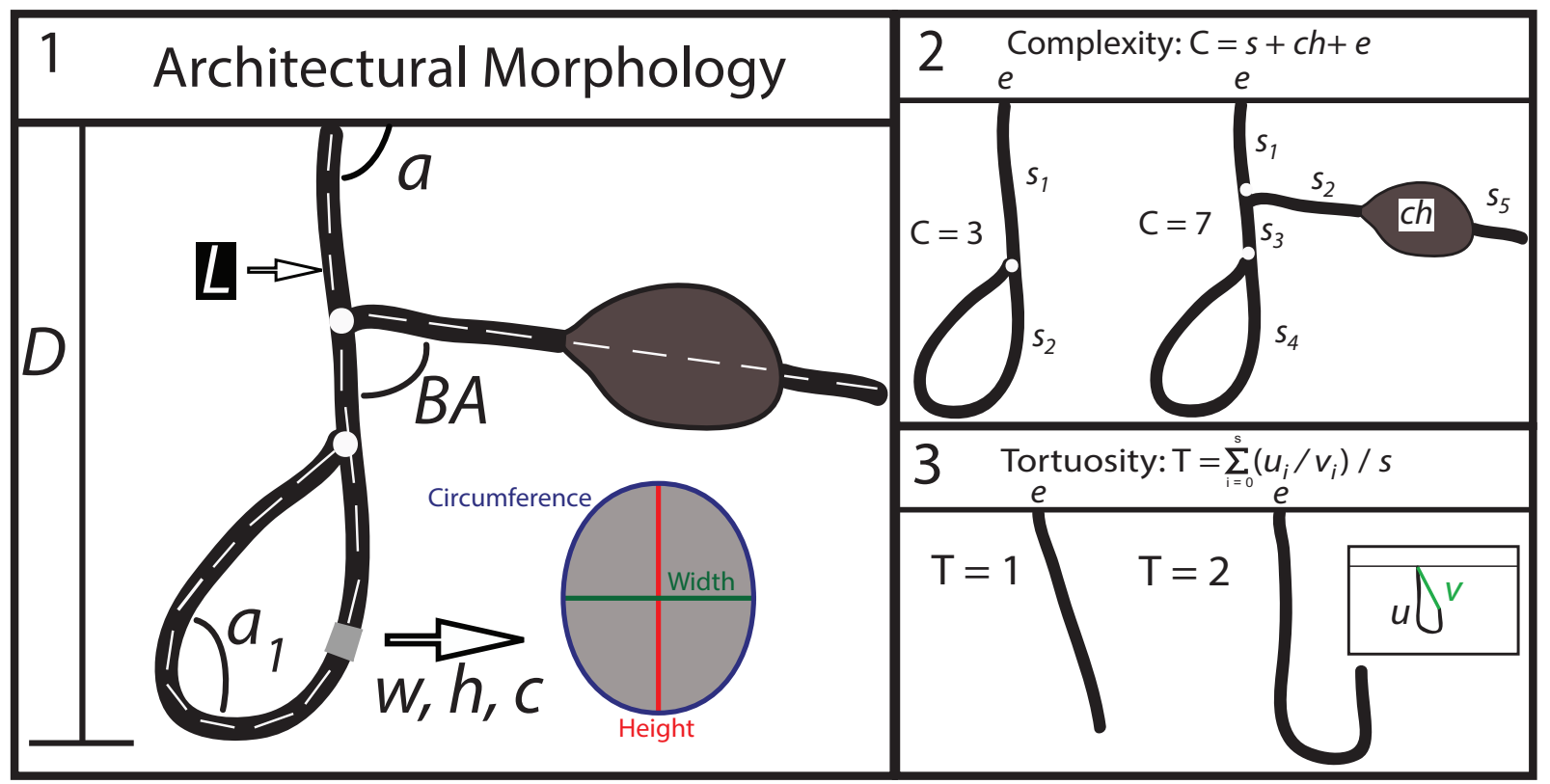

FIGURE 2. Diagrams of quantitative burrow measurements. 1. Burrows were described using the mean of the angle at which they entered the subsurface $(a)$ and the angle at which they shift below the surface $\left(a_{1}\right)$, total length $(L)$, branching angle $(B A)$, maximum depth below the surface $(D)$, shaft and chamber width $(w)$, height $(h)$, and circumference (c). 2. The complexity of a burrow system is the sum of the number of entrances $(e)$, segments $(s)$, and chambers (ch). 3. The tortuosity of a burrow system is the mean sinuosity of all burrow segments in the system. A single segment's tortuosity is the total length of the segment $(u)$ divided by the straight line distance $(v)$.

sediment moisture was obtained by mixing the sediment with water and measuring the moisture content with an Aquaterr EC-300 Salinity Multimeter every $10 \mathrm{~cm}$ until the mean moisture throughout the terrarium reached the desired levels of $35 \%$, $50 \%$, and $60-70 \%$.

Throughout the experiments, the laboratory was kept at $26^{\circ} \mathrm{C}$ and $20 \%$ humidity with the lights set on a 12-hour light-dark cycle. In order to maintain moisture within the sediment, the sediment surface was sprayed with water five days per week. Moisture levels were checked weekly. The millipedes were fed apples placed on the sediment surface and filled water dishes were kept inside the terraria.

Millipedes were placed on the sediment surface with the laboratory lights on. The millipedes were monitored for up to one hour or until all individuals burrowed under the surface. Observations recorded included the description of surface locomotion structures, time to the onset of burrowing, burrowing techniques, and the total time needed to burrow below the surface. The terraria were then monitored several times daily for the appearance of new burrow openings, closed burrow openings, and behaviors associated with surface traces and burrows. After 7-14 days millipedes found on the surface were removed; this continued until all individuals surfaced or were removed by excavation (up to 94 days). If no open burrows were produced at the end of the experiment this result was recorded. Open burrows were cast with Drystone ${ }^{\circledR}$ plaster, excavated by hand, and described.

Burrow casts were described using qualitative descriptions of their general morphology and surficial markings as well as 10 quantitative properties. Eight quantitative measurements (Figure 2.1) included: 1) number of surface openings, 2) maximum depth, 3) total length, 4) width, 5) height, 6) width-to-height ratio, 7) circumference, and 8) the slope relative to the sediment surface. Two derived properties included: 9) complexity (Figure 2.2) and 10) tortuosity (Figure 2.3).

The maximum depth was measured perpendicular to the surface from the entrance to the deepest portion of the burrow. The total length included the sum of the lengths of all the shafts, tunnels, and chambers in the burrow system. The width, height, width-to-height ratio, circumference, and slope of the shafts, tunnels, and chambers were measured every $1 \mathrm{~cm}$ starting from the surface opening. The mean value of each property was calculated to obtain a single representative measurement. Complexity and tortuosity are two 
scale independent properties of burrows (Meadows, 1991; Hembree and Hasiotis, 2006). Complexity is a measure of the number of different elements within a burrow and is determined by taking the sum of the number of surface openings, segments, and chambers (Figure 2.2). Tortuosity is a measure of the deviation of a burrow shaft or tunnel from a straight line (Figure 2.3). It is calculated by dividing the total length of a shaft or tunnel by the shortest distance between the endpoints. In burrows with two or more intersecting shafts or tunnels, the total tortuosity is the mean of the tortuosity of each element.

The 10 quantitative properties were used to compare the burrows of Narceus americanus and Floridobolus penneri both within and between each species. In addition, the burrows of these species were compared to those of two spirostreptid millipedes, Orthoporus ornatus and Archispirostreptus gigas from the southwestern United States and western Africa, respectively, produced in a previous study using similar experimental methods (Hembree, 2009). The burrow casts were compared using the non-parametric Bray Curtis similarity measure. This statistical test allows each burrow to be compared using all 10 quantitative variables at once to determine the level of similarity between each burrow cast (Hammer and Harper, 2006; Hembree et al., 2012). The output of this test is a similarity value between $0-1$, with 0 indicating complete difference and 1 indicating complete similarity. This study follows designations made in Hembree et al. (2012) that interpret values from $0.9-0.8$ as indicating a high level of similarity, 0.70.6 as indicating a moderate level of similarity, and any value from 0.5 to 0 as indicating dissimilarity. The results of the Bray Curtis test were summarized with cluster analyses using an unweighted paired group method.

In order to evaluate the effect of differences in environmental conditions on burrow morphology, Spearman's rank correlation was used to compare sediment properties and burrow properties. This nonparametric test is used to determine if two variables are covariant (Hammer and Harper, 2006). In this test, the two variables are converted to ranks and then a correlation test is run using the ranked data. Sediment composition and sediment moisture content were treated as independent variables while the 10 quantitative burrow properties were treated as dependent variables. This test produces a value (Rs) from 1.00 to -1.00 . Positive Rs values indicate that a burrow property increased with increases in the sediment property being mea- sured. Negative Rs values indicate an inverse relationship. Spearman's Rs values $>0.8$ or $<-0.8$ indicate a significant positive or negative correlation, respectively. All statistical tests were carried out with Paleontological Statistics (PAST ver. 2.16) (Hammer et al., 2001).

\section{EXPERIMENTAL RESULTS}

Both Narceus americanus and Floridobolus penneri produced temporarily and permanently open burrows and surface traces in all sediment compositions and moisture conditions. A total of 29 and 42 burrows were successfully cast from $N$. americanus and F. penneri, respectively (Tables 2, 3 ). Five basic burrow architectures were produced with four modifying features (Figure 3).

\section{Behavior}

Narceus americanus. Individuals began burrowing within 0-15 minutes after being placed into the terraria. Some initial burrows were abandoned and not completed. In sediments without sand, Narceus americanus used a compaction technique of burrowing (Appendix 1). The millipedes rammed their heads into the sediment using the collum like a plow. After moving forward the length of 3-4 segments, the millipede lifted its head and pushed upward to compact the displaced sediment into the roof of the burrow. The millipede then pushed forward, deeper into the sediment, and repeated the process. In sandy sediment, excavation was used to start the burrow (Appendix 2). The millipedes used their mandibles and front legs to remove sediment grains from the burrow walls and pushed them farther along the length of their body until the grains were deposited at the surface. Excavation was used until an entry into the sediment was established and was then followed by compaction burrowing. Burrowing completely below the surface took 5-60 minutes (Figure 4). Individuals were found up to $26 \mathrm{~cm}$ below the surface. Molting occurred in chambers $10 \mathrm{~cm}$ below the surface. The millipedes did return to the surface to feed, but most of this activity was observed in the dark hours.

Floridobolus penneri. Individuals started burrowing within 30 minutes of the start of the trials. Some initial burrows were abandoned leaving only a small divot in the sediment surface. Floridobolus penneri used a compaction burrowing technique at the surface, regardless of sediment type (Appendix 3 ). In the subsurface, however, F. penneri used an excavation technique, first removing sediment grains with their mandibles and then moving them 
BOWEN \& HEMBREE: NEOICHNOLOGY OF SPIROBOLIDS

TABLE 2. Quantitative measurements of burrow casts produced by Narceus americanus.

\begin{tabular}{|c|c|c|c|c|c|c|c|c|c|c|c|c|c|}
\hline & NA32 & NA23 & NA25A & NA21A & NA21B & NA21C & NA30B & NA30D & NA21D & NA24 & NA21E & NA26A & NA30E \\
\hline Architecture & sv & sv & sv & sv & sv & sv & sv & sv & SVb & SVc & SVc & SVc & SVc \\
\hline Surface Openings & 1 & 1 & 1 & 1 & 1 & 1 & 1 & 1 & 1 & 1 & 1 & 1 & 1 \\
\hline $\begin{array}{l}\text { Maximum Depth } \\
\text { (cm) }\end{array}$ & 4.1 & 6.7 & 3.0 & 1.9 & 4.0 & 7.1 & 2.6 & 5.9 & 11.9 & 4.0 & 13.4 & 5.8 & 6.6 \\
\hline Total Length (cm) & 4.3 & 8.2 & 9.4 & 2.3 & 4.3 & 8.0 & 3.1 & 6.8 & 13.8 & 7.2 & 15.7 & 6.8 & 7.5 \\
\hline $\begin{array}{l}\text { Maximum Width } \\
(\mathrm{mm})\end{array}$ & 9.3 & 16.9 & 11.7 & 13.0 & 14.5 & 11.5 & 9.6 & 10.8 & 11.7 & 17.6 & 16.8 & 26.2 & 16.5 \\
\hline $\begin{array}{l}\text { Minimum Width } \\
(\mathrm{mm})\end{array}$ & 8.5 & 8.0 & 7.8 & 10.5 & 11.0 & 8.8 & 9.5 & 7.5 & 8.6 & 7.1 & 7.2 & 11.2 & 6.0 \\
\hline $\begin{array}{l}\text { Average Width } \\
(\mathrm{mm})\end{array}$ & 8.8 & 10.4 & 10.2 & 11.8 & 12.4 & 9.3 & 9.6 & 9.3 & 10.2 & 10.9 & 10.5 & 15.6 & 11.4 \\
\hline $\begin{array}{l}\text { Maximum Height } \\
(\mathrm{mm})\end{array}$ & 9.8 & 9.5 & 10.1 & 12.5 & 12.3 & 8.8 & 9.8 & 10.9 & 11.6 & 9.7 & 10.4 & 16.4 & 10.0 \\
\hline $\begin{array}{l}\text { Minimum Height } \\
(\mathrm{mm})\end{array}$ & 8.6 & 7.3 & 5.7 & 11.8 & 10.5 & 7.4 & 8.2 & 8.1 & 6.3 & 6.0 & 5.8 & 8.9 & 7.0 \\
\hline $\begin{array}{l}\text { Average Height } \\
(\mathrm{mm})\end{array}$ & 9.2 & 8.3 & 7.9 & 12.2 & 11.4 & 8.1 & 9.0 & 9.4 & 9.3 & 8.0 & 9.1 & 11.6 & 8.4 \\
\hline Average W/H Ratio & 1.0 & 1.3 & 1.3 & 1.0 & 1.1 & 1.1 & 1.1 & 1.0 & 1.1 & 1.3 & 1.2 & 1.4 & 1.3 \\
\hline $\begin{array}{l}\text { Maximum } \\
\text { Circumference } \\
\text { (cm) }\end{array}$ & 3 & 4.1 & 3.7 & 4.2 & 4.3 & 3.1 & 2.9 & 3.5 & 3.6 & 4.6 & 4.3 & 8.4 & 4.7 \\
\hline $\begin{array}{l}\text { Minimum } \\
\text { Circumference } \\
\text { (cm) }\end{array}$ & 2.7 & 2.7 & 2.5 & 3.5 & 3.3 & 2.2 & 2.8 & 2.7 & 2.6 & 2.5 & 2.3 & 3.3 & 2.5 \\
\hline $\begin{array}{l}\text { Average } \\
\text { Circumference } \\
\text { (cm) }\end{array}$ & 2.8 & 3.1 & 3.1 & 3.9 & 3.7 & 2.7 & 2.9 & 3.1 & 3.1 & 3.3 & 3.2 & 4.9 & 3.5 \\
\hline $\begin{array}{l}\text { Maximum Slope } \\
\text { (Degrees) }\end{array}$ & 70 & 65 & 45 & 33 & 73 & 90 & 70 & 80 & 90 & 67 & 81 & 55 & 90 \\
\hline $\begin{array}{l}\text { Minimum Slope } \\
\text { (Degrees) }\end{array}$ & 70 & 50 & 25 & 33 & 70 & 25 & 70 & 60 & 40 & 20 & 55 & 55 & 65 \\
\hline $\begin{array}{l}\text { Average Slope } \\
\text { (Degrees) }\end{array}$ & 70 & 38 & 35 & 33 & 72 & 57 & 70 & 70 & 63 & 44 & 64 & 55 & 78 \\
\hline $\begin{array}{l}\text { Branching Angle } \\
\text { (Degrees) }\end{array}$ & $\mathrm{n} / \mathrm{a}$ & $\mathrm{n} / \mathrm{a}$ & $\mathrm{n} / \mathrm{a}$ & $\mathrm{n} / \mathrm{a}$ & $\mathrm{n} / \mathrm{a}$ & $\mathrm{n} / \mathrm{a}$ & $\mathrm{n} / \mathrm{a}$ & $\mathrm{n} / \mathrm{a}$ & 60 & $\mathrm{n} / \mathrm{a}$ & $\mathrm{n} / \mathrm{a}$ & $\mathrm{n} / \mathrm{a}$ & $\mathrm{n} / \mathrm{a}$ \\
\hline Complexity & 2.0 & 2.0 & 2.0 & 2.0 & 2.0 & 2.0 & 2.0 & 2.0 & 4.0 & 3.0 & 4.0 & 3.0 & 4.0 \\
\hline Tortuosity & 1.0 & 1.2 & 1.7 & 1.0 & 1.1 & 1.1 & 1.2 & 1.2 & 1.1 & 1.5 & 1.1 & 1.1 & 1.1 \\
\hline
\end{tabular}


TABLE 2 (continued).

\begin{tabular}{|c|c|c|c|c|c|c|c|c|c|c|c|c|}
\hline & NA28 & NA29C & NA30A & NA30C & NA33A & NA33B & NA30F & NA1 & NA25B & NA33C & NA29B & NA21F \\
\hline Architecture & $\mathbf{v}$ & V & v & $\mathbf{V}$ & $\mathbf{v}$ & V & Vh & $\mathbf{H}$ & $\mathbf{H}$ & $\mathbf{H}$ & $\mathrm{Hb}$ & $\mathrm{Hc}$ \\
\hline Surface Openings & 1 & 1 & 1 & 1 & 1 & 1 & 1 & 1 & 1 & 1 & 1 & 1 \\
\hline $\begin{array}{l}\text { Maximum Depth } \\
(\mathrm{cm})\end{array}$ & 2.4 & 1.9 & 2.5 & 2.3 & 3.1 & 2.2 & 5.9 & 2.8 & 2.5 & 3.0 & 3.4 & 2.9 \\
\hline Total Length (cm) & 3.0 & 1.9 & 2.5 & 2.6 & 3.1 & 3.0 & 11.8 & 7.0 & 8.4 & 6.8 & 10.7 & 10.0 \\
\hline $\begin{array}{l}\text { Maximum Width } \\
(\mathrm{mm})\end{array}$ & 14.0 & 11.7 & 9.3 & 10.4 & 12.0 & 13.8 & 10.4 & 10.2 & 11.0 & 11.8 & 10.6 & 16.5 \\
\hline $\begin{array}{l}\text { Minimum Width } \\
(\mathrm{mm})\end{array}$ & 14.0 & 11.7 & 7.0 & 9.3 & 10.4 & 13.0 & 7.2 & 7.4 & 9.0 & 7.0 & 7.4 & 6.8 \\
\hline $\begin{array}{l}\text { Average Width } \\
(\mathrm{mm})\end{array}$ & 14.0 & 11.7 & 8.2 & 9.9 & 11.2 & 13.4 & 9.1 & 8.9 & 10.1 & 9.6 & 8.4 & 11.6 \\
\hline $\begin{array}{l}\text { Maximum Height } \\
\text { (mm) }\end{array}$ & 13.8 & 9.0 & 8.8 & 9.3 & 14.7 & 14.1 & 9.6 & 9.8 & 9.1 & 11.7 & 9.2 & 11.5 \\
\hline $\begin{array}{l}\text { Minimum Height } \\
(\mathrm{mm})\end{array}$ & 9.5 & 9.0 & 7.7 & 8.0 & 11.1 & 10.6 & 6.7 & 6.3 & 5.8 & 8.5 & 6.1 & 8.0 \\
\hline $\begin{array}{l}\text { Average Height } \\
(\mathrm{mm})\end{array}$ & 11.7 & 9.0 & 8.3 & 8.7 & 12.9 & 12.4 & 8.3 & 8.3 & 8.0 & 9.8 & 7.8 & 9.7 \\
\hline Average W/H Ratio & 1.2 & 1.3 & 1.0 & 1.1 & 0.9 & 1.1 & 1.1 & 1.1 & 1.3 & 1.0 & 1.1 & 1.2 \\
\hline $\begin{array}{l}\text { Maximum } \\
\text { Circumference } \\
\text { (cm) }\end{array}$ & 5 & 3.5 & 2.8 & 3.1 & 4.3 & 4.4 & 3.2 & 3.2 & 3.3 & 4.3 & 3 & 4.3 \\
\hline $\begin{array}{l}\text { Minimum } \\
\text { Circumference } \\
\text { (cm) }\end{array}$ & 3.7 & 3.5 & 2.4 & 2.8 & 3.5 & 3.9 & 2.1 & 2.2 & 3 & 2.3 & 2.5 & 2.3 \\
\hline $\begin{array}{l}\text { Average } \\
\text { Circumference } \\
\text { (cm) }\end{array}$ & 4.4 & 3.5 & 2.6 & 3.0 & 3.9 & 4.2 & 2.8 & 2.8 & 3.2 & 3.1 & 2.7 & 3.4 \\
\hline $\begin{array}{l}\text { Maximum Slope } \\
\text { (Degrees) }\end{array}$ & 85 & 80 & 90 & 80 & 90 & 90 & 90 & 60 & 50 & 80 & 60 & 55 \\
\hline $\begin{array}{l}\text { Minimum Slope } \\
\text { (Degrees) }\end{array}$ & 85 & 80 & 90 & 80 & 90 & 90 & 0 & 15 & 0 & 50 & 60 & 0 \\
\hline $\begin{array}{l}\text { Average Slope } \\
\text { (Degrees) }\end{array}$ & 85 & 80 & 90 & 80 & 90 & 90 & 45 & 45 & 25 & 65 & 75 & 25 \\
\hline $\begin{array}{l}\text { Branching Angle } \\
\text { (Degrees) }\end{array}$ & $\mathrm{n} / \mathrm{a}$ & $\mathrm{n} / \mathrm{a}$ & $\mathrm{n} / \mathrm{a}$ & $\mathrm{n} / \mathrm{a}$ & $\mathrm{n} / \mathrm{a}$ & $\mathrm{n} / \mathrm{a}$ & $\mathrm{n} / \mathrm{a}$ & $\mathrm{n} / \mathrm{a}$ & $\mathrm{n} / \mathrm{a}$ & $\mathrm{n} / \mathrm{a}$ & 90 & $\mathrm{n} / \mathrm{a}$ \\
\hline Complexity & 2.0 & 2.0 & 2.0 & 2.0 & 2.0 & 2.0 & 2.0 & 2.0 & 2.0 & 2.0 & 4.0 & 4.0 \\
\hline Tortuosity & 1.3 & 1.0 & 1.0 & 1.1 & 1.0 & 1.0 & 2.0 & 3.5 & 5.6 & 2.3 & 1.5 & 1.2 \\
\hline
\end{tabular}


TABLE 2 (continued).

\begin{tabular}{|c|c|c|c|c|c|}
\hline & NA29A & NA26B & NA31A & NA31B & $\begin{array}{l}\text { Total } \\
\text { Mean }\end{array}$ \\
\hline Architecture & $\mathrm{O}(\mathrm{T} 1)$ & $\mathrm{O}(\mathrm{T} 1)$ & $\mathrm{O}(\mathrm{T} 2)$ & $\mathrm{O}(\mathrm{T} 2)$ & \\
\hline Surface Openings & 1 & 1 & 2 & 1 & 1 \\
\hline $\begin{array}{l}\text { Maximum Depth } \\
\text { (cm) }\end{array}$ & 3.4 & 6.9 & 2.5 & 1.9 & 4.4 \\
\hline Total Length (cm) & 10.6 & 12.5 & 12.3 & 10.0 & 7.4 \\
\hline $\begin{array}{l}\text { Maximum Width } \\
(\mathrm{mm})\end{array}$ & 12.9 & 18.0 & 13.4 & 10.9 & 13.2 \\
\hline $\begin{array}{l}\text { Minimum Width } \\
(\mathrm{mm})\end{array}$ & 8.3 & 7.4 & 8.7 & 9.1 & 8.8 \\
\hline Average Width (mm) & 10.5 & 10.9 & 10.4 & 10.1 & 10.6 \\
\hline $\begin{array}{l}\text { Maximum Height } \\
(\mathrm{mm})\end{array}$ & 9.6 & 11.3 & 12.7 & 11.7 & 11.0 \\
\hline $\begin{array}{l}\text { Minimum Height } \\
(\mathrm{mm})\end{array}$ & 6.0 & 6.7 & 9.3 & 8.9 & 7.9 \\
\hline $\begin{array}{l}\text { Average Height } \\
(\mathrm{mm})\end{array}$ & 8.2 & 9.3 & 10.4 & 9.9 & 9.5 \\
\hline Average W/H Ratio & 1.3 & 1.2 & 1.0 & 1.0 & 1.1 \\
\hline $\begin{array}{l}\text { Maximum } \\
\text { Circumference }(\mathrm{cm})\end{array}$ & 3.5 & 4.8 & 3.9 & 3.6 & 4.0 \\
\hline $\begin{array}{l}\text { Minimum } \\
\text { Circumference }(\mathrm{cm})\end{array}$ & 2.7 & 2.3 & 2.9 & 3.1 & 2.8 \\
\hline $\begin{array}{l}\text { Average } \\
\text { Circumference }(\mathrm{cm})\end{array}$ & 3.1 & 3.4 & 3.5 & 3.3 & 3.3 \\
\hline $\begin{array}{l}\text { Maximum Slope } \\
\text { (Degrees) }\end{array}$ & 60 & 60 & 90 & 20 & 71 \\
\hline $\begin{array}{l}\text { Minimum Slope } \\
\text { (Degrees) }\end{array}$ & 15 & 60 & 0 & 20 & 47 \\
\hline $\begin{array}{l}\text { Average Slope } \\
\text { (Degrees) }\end{array}$ & 38 & 60 & 43 & 20 & 59 \\
\hline $\begin{array}{l}\text { Branching Angle } \\
\text { (Degrees) }\end{array}$ & $\mathrm{n} / \mathrm{a}$ & $\mathrm{n} / \mathrm{a}$ & $\mathrm{n} / \mathrm{a}$ & $\mathrm{n} / \mathrm{a}$ & 75 \\
\hline Complexity & 3.0 & 3.0 & 6.0 & 2.0 & 2.6 \\
\hline Tortuosity & 43.0 & 39.0 & 1.8 & 99.0 & 7.6 \\
\hline
\end{tabular}


TABLE 3. Quantitative measurements of burrow casts produced by Floridobolus penneri.

\begin{tabular}{|c|c|c|c|c|c|c|c|c|c|c|c|c|}
\hline Architecture & $\begin{array}{c}\text { FP2C } \\
\text { SV }\end{array}$ & $\begin{array}{c}\text { FP3C } \\
\text { SV }\end{array}$ & $\begin{array}{c}\text { FP3D } \\
\text { SV }\end{array}$ & $\begin{array}{c}\text { FP3E } \\
\text { SV }\end{array}$ & $\begin{array}{l}\text { FP3F } \\
\text { SV }\end{array}$ & $\begin{array}{c}\text { FP3G } \\
\text { SV }\end{array}$ & $\begin{array}{c}\text { FP3H } \\
\text { SV }\end{array}$ & $\begin{array}{l}\text { FP3I } \\
\text { SV }\end{array}$ & $\begin{array}{c}\text { FP2E } \\
\text { SV }\end{array}$ & $\begin{array}{l}\text { FP2F } \\
\text { SV }\end{array}$ & $\begin{array}{c}\text { FP2G } \\
\text { SV }\end{array}$ & $\begin{array}{l}\text { FP6A } \\
\text { SV }\end{array}$ \\
\hline Surface Openings & 1 & 1 & 1 & 1 & 1 & 1 & 1 & 1 & 1 & 1 & 1 & 1 \\
\hline $\begin{array}{l}\text { Maximum Depth } \\
(\mathrm{cm})\end{array}$ & 3.5 & 1.7 & 3.3 & 3.3 & 3.6 & 6.8 & 6.5 & 8.2 & 7.9 & 6.9 & 4.4 & 1.8 \\
\hline Total Length (cm) & 4.0 & 1.7 & 4.2 & 3.3 & 4.2 & 7.4 & 6.8 & 8.6 & 9.6 & 9.5 & 10.0 & 4.3 \\
\hline $\begin{array}{l}\text { Maximum Width } \\
(\mathrm{mm})\end{array}$ & 13.1 & 9.4 & 14.0 & 14.1 & 14.2 & 10.8 & 12.8 & 12.8 & 12.5 & 12.2 & 6.5 & 17.0 \\
\hline $\begin{array}{l}\text { Minimum Width } \\
(\mathrm{mm})\end{array}$ & 11.0 & 9.4 & 9.6 & 13.2 & 12.4 & 8.8 & 10.2 & 10.3 & 9.2 & 9.7 & 0.9 & 10.0 \\
\hline Average Width $(\mathrm{mm})$ & 12.1 & 9.4 & 11.1 & 13.7 & 13.2 & 10.1 & 11.4 & 11.4 & 11.0 & 10.8 & 4.0 & 13.7 \\
\hline $\begin{array}{l}\text { Maximum Height } \\
(\mathrm{mm})\end{array}$ & 14.3 & 10.6 & 11.6 & 12.0 & 11.1 & 12.0 & 12.4 & 12.5 & 13.3 & 11.4 & 7.8 & 10.7 \\
\hline $\begin{array}{l}\text { Minimum Height } \\
(\mathrm{mm})\end{array}$ & 10.0 & 10.6 & 8.6 & 8.3 & 9.2 & 8.7 & 10.8 & 8.5 & 8.1 & 8.7 & 3.4 & 10.0 \\
\hline $\begin{array}{l}\text { Average Height } \\
(\mathrm{mm})\end{array}$ & 12.8 & 10.6 & 10.0 & 10.2 & 10.4 & 10.5 & 11.5 & 10.5 & 10.9 & 10.1 & 5.8 & 10.3 \\
\hline Average W/H Ratio & 1.0 & 0.9 & 1.1 & 1.4 & 1.3 & 1.0 & 1.0 & 1.1 & 1.0 & 1.1 & 0.7 & 1.3 \\
\hline $\begin{array}{l}\text { Maximum } \\
\text { Circumference (cm) }\end{array}$ & 4.4 & 3.2 & 4 & 4.4 & 4 & 3.7 & 4 & 4.1 & 3.9 & 3.7 & 3.7 & 4.5 \\
\hline $\begin{array}{l}\text { Minimum } \\
\text { Circumference (cm) }\end{array}$ & 3.4 & 3.2 & 3.1 & 3.7 & 3.8 & 2.9 & 3.1 & 3.2 & 3.2 & 2.9 & 2.4 & 2.8 \\
\hline $\begin{array}{l}\text { Average } \\
\text { Circumference }(\mathrm{cm})\end{array}$ & 4.0 & 3.2 & 3.4 & 4.1 & 3.9 & 3.3 & 3.6 & 3.6 & 3.6 & 3.4 & 3.2 & 3.7 \\
\hline $\begin{array}{l}\text { Maximum Slope } \\
\text { (Degrees) }\end{array}$ & 65 & 75 & 68 & 65 & 75 & 78 & 70 & 65 & 65 & 55 & 78 & 20 \\
\hline $\begin{array}{l}\text { Minimum Slope } \\
\text { (Degrees) }\end{array}$ & 65 & 75 & 68 & 65 & 75 & 78 & 70 & 65 & 65 & 55 & 78 & 20 \\
\hline $\begin{array}{l}\text { Average Slope } \\
\text { (Degrees) }\end{array}$ & 65 & 75 & 68 & 65 & 75 & 78 & 70 & 65 & 65 & 55 & 78 & 20 \\
\hline $\begin{array}{l}\text { Branching Angle } \\
\text { (Degrees) }\end{array}$ & $\mathrm{n} / \mathrm{a}$ & $\mathrm{n} / \mathrm{a}$ & $\mathrm{n} / \mathrm{a}$ & $\mathrm{n} / \mathrm{a}$ & $\mathrm{n} / \mathrm{a}$ & $\mathrm{n} / \mathrm{a}$ & $\mathrm{n} / \mathrm{a}$ & $\mathrm{n} / \mathrm{a}$ & $\mathrm{n} / \mathrm{a}$ & $\mathrm{n} / \mathrm{a}$ & $\mathrm{n} / \mathrm{a}$ & $\mathrm{n} / \mathrm{a}$ \\
\hline Complexity & 2.0 & 2.0 & 2.0 & 2.0 & 2.0 & 2.0 & 2.0 & 2.0 & 2.0 & 2.0 & 4.0 & 2.0 \\
\hline Tortuosity & 1.0 & 1.0 & 1.2 & 1.0 & 1.2 & 1.1 & 1.0 & 1.1 & 1.1 & 1.2 & 1.1 & 1.4 \\
\hline
\end{tabular}


BOWEN \& HEMBREE: NEOICHNOLOGY OF SPIROBOLIDS

TABLE 3 (continued).

\begin{tabular}{|c|c|c|c|c|c|c|c|c|c|c|}
\hline & FP3A & FP5B & FP5C & FP5D & FP7A & FP7C & FP7E & FP7F & FP7H & FP5E \\
\hline Architecture & SV & SV & SV & SV & SV & SV & SV & SVb & SVcb & SVc \\
\hline Surface Openings & 1 & 1 & 1 & 1 & 1 & 1 & 1 & 1 & 1 & 1 \\
\hline $\begin{array}{l}\text { Maximum Depth } \\
\text { (cm) }\end{array}$ & 1.5 & 2.8 & 4.2 & 5.7 & 2.1 & 4.1 & 8.7 & 8.0 & 7.4 & 5.2 \\
\hline Total Length (cm) & 3.3 & 3.4 & 4.9 & 7.7 & 2.4 & 5.5 & 9.2 & 12.4 & 18.6 & 6.8 \\
\hline $\begin{array}{l}\text { Maximum Width } \\
(\mathrm{mm})\end{array}$ & 8.2 & 15.9 & 12.9 & 16.3 & 11.3 & 16.0 & 15.0 & 18.3 & 30.1 & 16.4 \\
\hline $\begin{array}{l}\text { Minimum Width } \\
(\mathrm{mm})\end{array}$ & 7.3 & 14.5 & 8.0 & 12.7 & 10.3 & 9.1 & 9.0 & 10.1 & 8.7 & 10.4 \\
\hline $\begin{array}{l}\text { Average Width } \\
(\mathrm{mm})\end{array}$ & 7.8 & 15.2 & 11.0 & 13.9 & 10.8 & 12.8 & 12.3 & 13.6 & 16.8 & 12.7 \\
\hline $\begin{array}{l}\text { Maximum Height } \\
(\mathrm{mm})\end{array}$ & 8.5 & 19.6 & 11.8 & 16.3 & 12.0 & 14.0 & na & 12.8 & 19.0 & 24.7 \\
\hline $\begin{array}{l}\text { Minimum Height } \\
(\mathrm{mm})\end{array}$ & 7.6 & 13.3 & 10.9 & 11.8 & 10.8 & 10.7 & na & 10.3 & 8.7 & 11.5 \\
\hline $\begin{array}{l}\text { Average Height } \\
(\mathrm{mm})\end{array}$ & 8.1 & 16.5 & 11.5 & 13.9 & 11.4 & 12.7 & 10.0 & 11.3 & 13.4 & 15.6 \\
\hline Average W/H Ratio & 1.0 & 1.0 & 1.0 & 1.0 & 0.9 & 0.9 & 1.2 & 1.2 & 1.3 & 0.9 \\
\hline $\begin{array}{l}\text { Maximum } \\
\text { Circumference }(\mathrm{cm})\end{array}$ & 2.7 & 5.7 & 4.1 & 5.2 & 3.6 & 4.5 & 3.9 & 4.5 & 8.1 & 6.2 \\
\hline $\begin{array}{l}\text { Minimum } \\
\text { Circumference }(\mathrm{cm})\end{array}$ & 2.6 & 4.6 & 3.2 & 3.3 & 3.1 & 3 & 2.9 & 3.1 & 2.6 & 3.3 \\
\hline $\begin{array}{l}\text { Average } \\
\text { Circumference }(\mathrm{cm})\end{array}$ & 2.7 & 5.2 & 3.7 & 4.5 & 3.4 & 3.9 & 3.6 & 4.0 & 5.0 & 4.7 \\
\hline $\begin{array}{l}\text { Maximum Slope } \\
\text { (Degrees) }\end{array}$ & 45 & 45 & 70 & 55 & 75 & 75 & 90 & 90 & 90 & 65 \\
\hline $\begin{array}{l}\text { Minimum Slope } \\
\text { (Degrees) }\end{array}$ & 45 & 45 & 70 & 55 & 75 & 50 & 65 & 50 & 20 & 45 \\
\hline $\begin{array}{l}\text { Average Slope } \\
\text { (Degrees) }\end{array}$ & 45 & 45 & 70 & 55 & 75 & 62 & 78 & 70 & 50 & 55 \\
\hline $\begin{array}{l}\text { Branching Angle } \\
\text { (Degrees) }\end{array}$ & $\mathrm{n} / \mathrm{a}$ & $\mathrm{n} / \mathrm{a}$ & $\mathrm{n} / \mathrm{a}$ & $\mathrm{n} / \mathrm{a}$ & $\mathrm{n} / \mathrm{a}$ & $\mathrm{n} / \mathrm{a}$ & $\mathrm{n} / \mathrm{a}$ & 20 & 55 & $\mathrm{n} / \mathrm{a}$ \\
\hline Complexity & 2.0 & 2.0 & 2.0 & 2.0 & 2.0 & 2.0 & 2.0 & 3.0 & 4.0 & 3.0 \\
\hline Tortuosity & 1.6 & 1.1 & 1.1 & 1.2 & 1.1 & 1.3 & 1.1 & 1.1 & 1.5 & 1.3 \\
\hline
\end{tabular}


TABLE 3 (continued).

\begin{tabular}{|c|c|c|c|c|c|c|c|c|c|}
\hline & FP3B & FP2A & FP2B & FP2D & FP4A & FP5A & FP7B & FP2M & FP7D \\
\hline Architecture & $\mathbf{v}$ & $\mathbf{V}$ & $\mathbf{V}$ & $\mathbf{v}$ & $\mathbf{V}$ & v & $\mathbf{V}$ & $\mathbf{v}$ & Vh \\
\hline Surface Openings & 1 & 1 & 1 & 1 & 1 & 1 & 1 & 1 & 1 \\
\hline $\begin{array}{l}\text { Maximum Depth } \\
(\mathrm{cm})\end{array}$ & 2.2 & 4.1 & 3.1 & 6.0 & 3.3 & 2.1 & 3.6 & 3.6 & 6.9 \\
\hline Total Length (cm) & 2.2 & 4.1 & 3.1 & 6.7 & 3.6 & 2.1 & 4.7 & 3.6 & 8.4 \\
\hline $\begin{array}{l}\text { Maximum Width } \\
(\mathrm{mm})\end{array}$ & 12.6 & 10.6 & 15.0 & 15.6 & 16.5 & 16.9 & 14.5 & 23.3 & 14.4 \\
\hline $\begin{array}{l}\text { Minimum Width } \\
(\mathrm{mm})\end{array}$ & 9.8 & 9.2 & 13.6 & 8.6 & 12.3 & 10.3 & 7.6 & 12.1 & 10.3 \\
\hline Average Width (mm) & 11.2 & 9.9 & 14.3 & 13.2 & 14.7 & 13.6 & 12.0 & 16.6 & 12.1 \\
\hline $\begin{array}{l}\text { Maximum Height } \\
(\mathrm{mm})\end{array}$ & 9.7 & 11.9 & 15.8 & 13.3 & 13.4 & 15.4 & 13.7 & 23.0 & 11.3 \\
\hline $\begin{array}{l}\text { Minimum Height } \\
(\mathrm{mm})\end{array}$ & 9.1 & 10.0 & 14.0 & 9.6 & 10.5 & 12.0 & 11.6 & 12.7 & 9.8 \\
\hline $\begin{array}{l}\text { Average Height } \\
(\mathrm{mm})\end{array}$ & 9.4 & 11.1 & 14.9 & 11.5 & 12.2 & 13.7 & 12.7 & 16.7 & 11.0 \\
\hline Average W/H Ratio & 1.2 & 0.9 & 1.0 & 1.1 & 1.2 & 1.0 & 0.7 & 1.0 & 1.1 \\
\hline $\begin{array}{l}\text { Maximum } \\
\text { Circumference (cm) }\end{array}$ & 3.6 & 3.8 & 5.2 & 5.6 & 5.5 & 4.6 & 4 & 6.5 & 3.8 \\
\hline $\begin{array}{l}\text { Minimum } \\
\text { Circumference }(\mathrm{cm})\end{array}$ & 3 & 3.4 & 4.6 & 2.6 & 3.5 & 3.4 & 3.2 & 3.8 & 3.3 \\
\hline $\begin{array}{l}\text { Average } \\
\text { Circumference }(\mathrm{cm})\end{array}$ & 3.3 & 3.6 & 4.9 & 4.4 & 4.6 & 4.0 & 3.6 & 5.2 & 3.6 \\
\hline $\begin{array}{l}\text { Maximum Slope } \\
\text { (Degrees) }\end{array}$ & 90 & 85 & 90 & 85 & 90 & 90 & 90 & 90 & 90 \\
\hline $\begin{array}{l}\text { Minimum Slope } \\
\text { (Degrees) }\end{array}$ & 90 & 85 & 90 & 60 & 90 & 90 & 40 & 90 & 55 \\
\hline $\begin{array}{l}\text { Average Slope } \\
\text { (Degrees) }\end{array}$ & 90 & 85 & 90 & 73 & 90 & 90 & 65 & 90 & 73 \\
\hline $\begin{array}{l}\text { Branching Angle } \\
\text { (Degrees) }\end{array}$ & $\mathrm{n} / \mathrm{a}$ & $\mathrm{n} / \mathrm{a}$ & $\mathrm{n} / \mathrm{a}$ & $\mathrm{n} / \mathrm{a}$ & $\mathrm{n} / \mathrm{a}$ & $\mathrm{n} / \mathrm{a}$ & $\mathrm{n} / \mathrm{a}$ & $\mathrm{n} / \mathrm{a}$ & $\mathrm{n} / \mathrm{a}$ \\
\hline Complexity & 2.0 & 2.0 & 2.0 & 2.0 & 2.0 & 2.0 & 2.0 & 2.0 & 2.0 \\
\hline Tortuosity & 1.0 & 1.0 & 1.0 & 1.1 & 1.1 & 1.0 & 1.2 & 1.0 & 1.2 \\
\hline
\end{tabular}


TABLE 3 (continued).

\begin{tabular}{|c|c|c|c|c|c|c|c|c|c|c|c|c|}
\hline Architecture & $\begin{array}{c}\text { FP2I } \\
\text { J }\end{array}$ & $\begin{array}{l}\text { FP2J } \\
\quad J\end{array}$ & $\begin{array}{c}\text { FP2K } \\
\text { J }\end{array}$ & $\begin{array}{c}\text { FP2L } \\
\text { J }\end{array}$ & $\begin{array}{l}\text { FP7G } \\
\text { J }\end{array}$ & $\begin{array}{l}\text { FP2H } \\
\text { Jb }\end{array}$ & $\begin{array}{c}\text { FP4B } \\
\text { H }\end{array}$ & $\begin{array}{l}\mathrm{FP1} \\
\mathrm{Hb}\end{array}$ & $\begin{array}{c}\text { FP5F } \\
\text { Ob (T1) }\end{array}$ & $\begin{array}{c}\text { FP6B } \\
\text { Oc (T1) }\end{array}$ & $\begin{array}{c}\text { FP5G } \\
\text { Ob (T2) }\end{array}$ & $\begin{array}{l}\text { Total } \\
\text { Mean }\end{array}$ \\
\hline Surface Openings & 1 & 1 & 1 & 1 & 1 & 1 & 1 & 1 & 1 & 1 & 1 & 1 \\
\hline $\begin{array}{l}\text { Maximum Depth } \\
\text { (cm) }\end{array}$ & 6.5 & 7.8 & 8.1 & 2.9 & 8.4 & 5.8 & 3.1 & 2.8 & 7.7 & 4.4 & 1.1 & 4.8 \\
\hline Total Length (cm) & 10.7 & 11.9 & 11.8 & 7.5 & 14.7 & 13.0 & 11.1 & 11.1 & 17.5 & 13.0 & 10.1 & 7.6 \\
\hline $\begin{array}{l}\text { Maximum Width } \\
(\mathrm{mm})\end{array}$ & 11.5 & 16.6 & 12.4 & 11.4 & 13.0 & 13.0 & 13.6 & 13.5 & 12.5 & 24.8 & 11.9 & 14.4 \\
\hline $\begin{array}{l}\text { Minimum Width } \\
(\mathrm{mm})\end{array}$ & 8.4 & 9.3 & 9.5 & 7.9 & 9.0 & 7.2 & 10.0 & 7.5 & 9.1 & 10.0 & 9.0 & 9.7 \\
\hline Average Width (mm) & 9.9 & 12.0 & 11.0 & 9.4 & 11.3 & 10.8 & 11.6 & 9.8 & 10.8 & 17.7 & 10.2 & 11.9 \\
\hline $\begin{array}{l}\text { Maximum Height } \\
(\mathrm{mm})\end{array}$ & 13.0 & 16.5 & 14.8 & 12.1 & 15.7 & 14.7 & 11.4 & 7.0 & 13.7 & 16.0 & 11.2 & 13.5 \\
\hline $\begin{array}{l}\text { Minimum Height } \\
(\mathrm{mm})\end{array}$ & 9.2 & 9.2 & 10.3 & 7.4 & 10.4 & 7.7 & 7.5 & 5.1 & 10.0 & 7.7 & 6.2 & 9.5 \\
\hline $\begin{array}{l}\text { Average Height } \\
(\mathrm{mm})\end{array}$ & 10.7 & 11.8 & 13.0 & 9.5 & 12.4 & 11.0 & 10.0 & 6.1 & 12.2 & 12.1 & 8.3 & 11.4 \\
\hline Average W/H Ratio & 0.9 & 1.0 & 0.9 & 1.0 & 0.9 & 1.0 & 1.2 & 1.6 & 0.9 & 1.5 & 1.3 & 1.1 \\
\hline $\begin{array}{l}\text { Maximum } \\
\text { Circumference (cm) }\end{array}$ & 3.7 & 5.3 & 4.2 & 3.5 & 4.2 & 6.4 & 4.3 & 3.4 & 4.1 & 6.1 & 3.5 & 4.5 \\
\hline $\begin{array}{l}\text { Minimum } \\
\text { Circumference }(\mathrm{cm})\end{array}$ & 2.9 & 2.8 & 3 & 2.6 & 3.1 & 2.4 & 3.1 & 2.2 & 3.2 & 2.6 & 2.7 & 3.1 \\
\hline $\begin{array}{l}\text { Average } \\
\text { Circumference }(\mathrm{cm})\end{array}$ & 3.2 & 3.8 & 3.8 & 3.1 & 3.9 & 3.7 & 3.6 & 2.7 & 3.7 & 4.4 & 3.1 & 3.8 \\
\hline $\begin{array}{l}\text { Maximum Slope } \\
\text { (Degrees) }\end{array}$ & 80 & 90 & 55 & 55 & 75 & 65 & 70 & 50 & 85 & 90 & 20 & 72 \\
\hline $\begin{array}{l}\text { Minimum Slope } \\
\text { (Degrees) }\end{array}$ & 15 & 50 & 50 & 45 & 75 & 25 & 0 & 25 & 85 & 40 & 20 & 58 \\
\hline $\begin{array}{l}\text { Average Slope } \\
\text { (Degrees) }\end{array}$ & 47 & 70 & 53 & 50 & 75 & 45 & 35 & 38 & 85 & 65 & 20 & 65 \\
\hline $\begin{array}{l}\text { Branching Angle } \\
\text { (Degrees) }\end{array}$ & $\mathrm{n} / \mathrm{a}$ & $\mathrm{n} / \mathrm{a}$ & $\mathrm{n} / \mathrm{a}$ & $\mathrm{n} / \mathrm{a}$ & $\mathrm{n} / \mathrm{a}$ & 70 & $\mathrm{n} / \mathrm{a}$ & 10,45 & 12,25 & $\mathrm{n} / \mathrm{a}$ & 10 & 31 \\
\hline Complexity & 2.0 & 2.0 & 2.0 & 2.0 & 2.0 & 3.0 & 2.0 & 6.0 & 6.0 & 4.0 & 4.0 & 2.5 \\
\hline Tortuosity & 2.2 & 2.0 & 2.0 & 5.8 & 2.4 & 1.4 & 4.3 & 1.1 & 17.6 & 53.0 & 1.4 & 3.1 \\
\hline
\end{tabular}




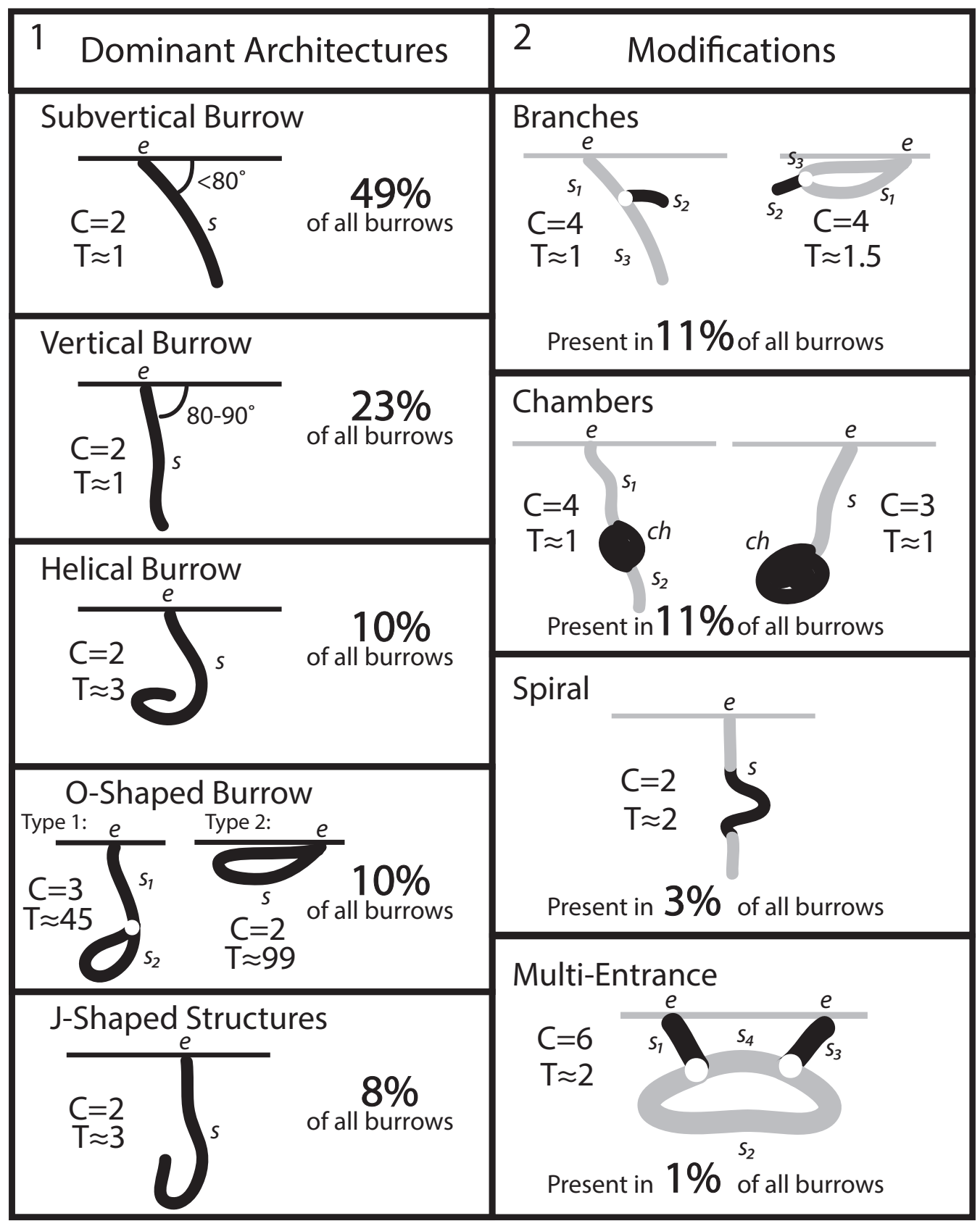

FIGURE 3. Dominant burrow architectures and modifications produced by Narceus americanus and Floridobolus penneri. Percents given include burrows produced by both $N$. americanus and $F$. penneri. Complexity $(\mathrm{C})$ and tortuosity $(\mathrm{T})$ are the average values for each burrow type. 1. Dominant architectures produced by the millipedes. 2. Modifications to dominant architectures that affect the quantitative properties. Black portions of the illustration represent the modifying structures while gray portions represent the modified dominant architecture.

along several diplosegments until they were deposited (Appendix 4). The millipedes rotated their body within the burrow to excavate along all sides. In this way F. penneri was able to excavate tunnels larger than its body. Burrows were from 6$33 \mathrm{~cm}$ deep (Figure 5.1). Molting was observed both at the surface (Figure 5.2) and in subsurface chambers. During long periods of inactivity, individ- uals were $2-40 \mathrm{~cm}$ below the surface. When exiting burrows, the millipedes typically emerged backwards, suggesting that there were no turnaround points in the burrow. Surface activity was most common during the laboratory's dark hours; however, some individuals did emerge in the light.

Both species either used the same burrow multiple times or attempted to enter a burrow that 


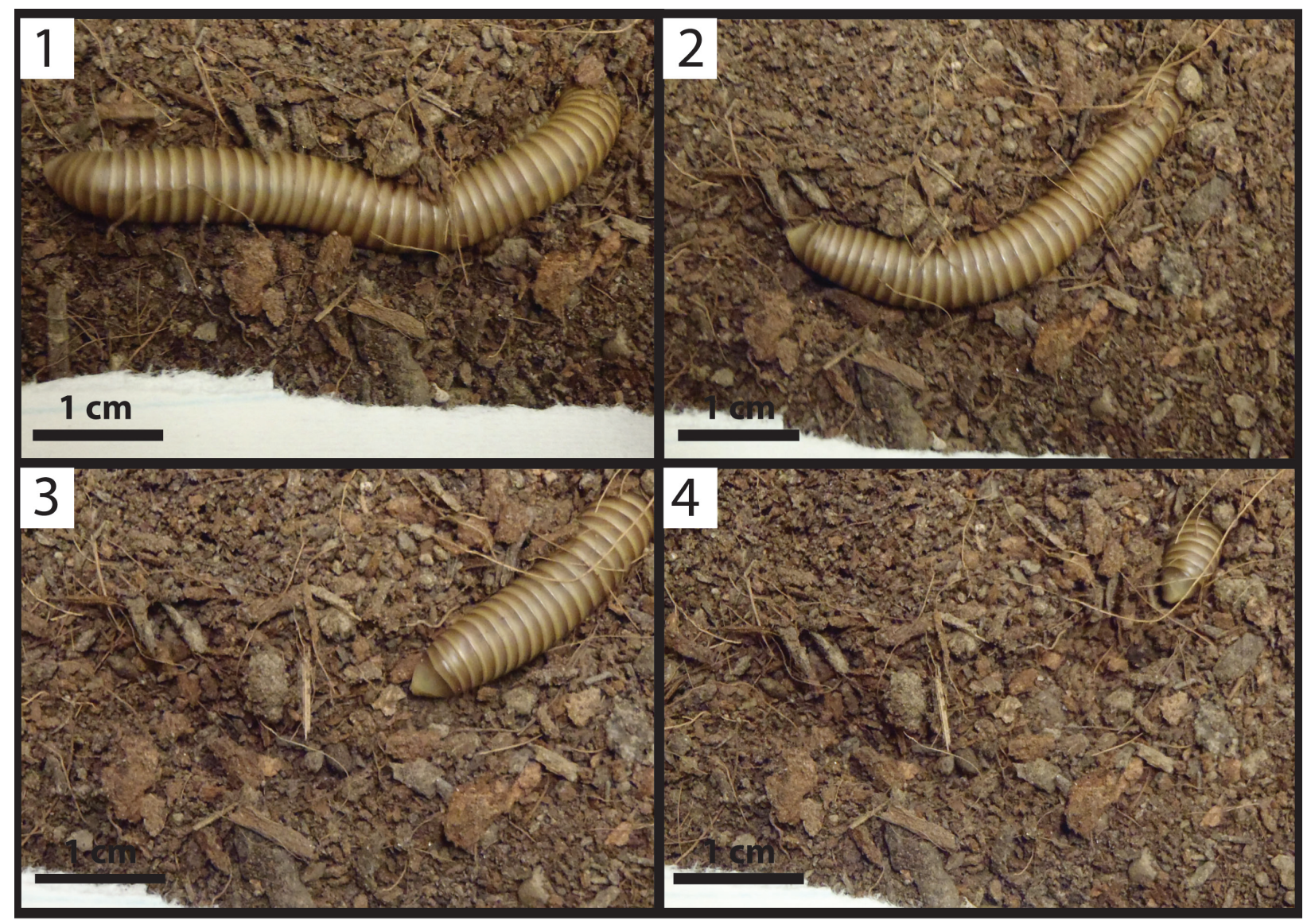

FIGURE 4. Time lapse photographs of Narceus americanus burrowing in 500/50S sediment with $35 \%$ moisture content. The photos are taken at approximately two minute intervals.

was created by another individual, even when it was still occupied. In many experiments, burrow openings collapsed while the individual was still deep within the sediment. During emergence, these millipedes produced a new burrow and surface opening. Occupation of the burrows lasted from several hours to over 90 days at which point those individuals were excavated. Surface activity of all individuals showed a marked increase after February 10. Of the 13 experiments conducted between August 15 and February 1, only 38\% $(n=5)$ were completed within 5 days of the expected experimental end date. Of the other 10 experiments (June 6 to August 8), 90\% ( $n=9$ ) were completed within 5 days of the expected end date.

\section{Surface Trace Morphology}

At the start of each experiment, the sediment surface was leveled and had a homogenous grain distribution. Surface traces consisted of furrows and divots made while attempting to burrow, locomotion trails, and burrow openings. The furrows were sinuous to straight and were generally char- acterized by a shallow trenching of the sediment surface along a semi-continuous path (Figure 6.12 ). Divots created by attempted burrowing were $0.1-0.7 \mathrm{~cm}$ deep, circular to elliptical indentations in the sediment surface. Locomotion trails were linear paths defined by ridges of large sediment grains on either side of a slight depression (Figure 6.3-4). Burrow openings were typically circular in shape, entered the surface from $5-90^{\circ}$, and had diameters similar to that of the producer (Figure 6.5-6). Small mounds of sediment were observed near burrow entrances where excavation was employed.

\section{Burrow Morphology}

The millipedes produced five basic burrow architectures including vertical, subvertical, Jshaped, helical, and O-shaped burrows (Figure 3.1). In addition to these base architectures, four modifying features were produced including branches, chambers, partial spirals, and additional entrances (Figure 3.2). The shafts, tunnels, and chambers produced by both species were circular 


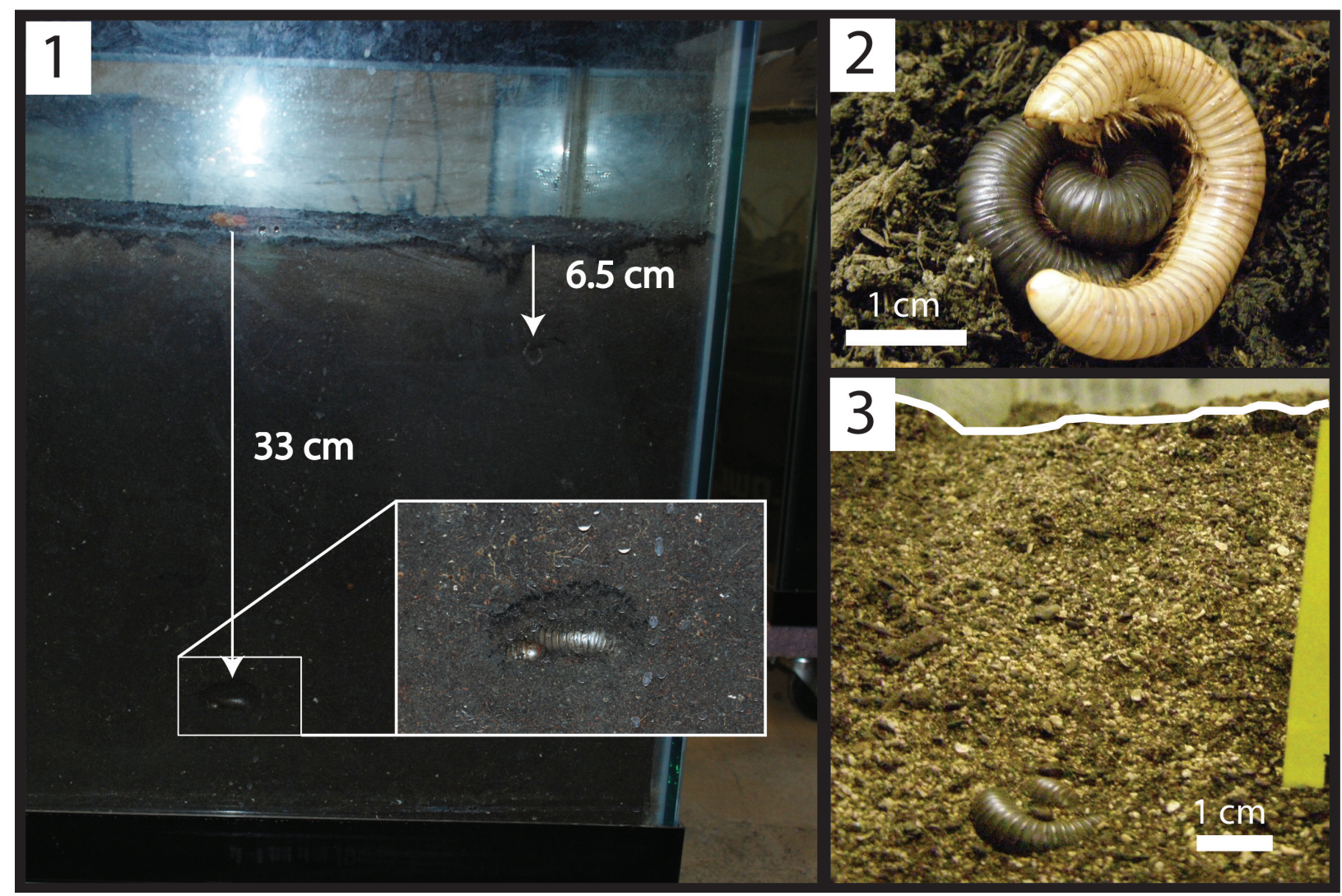

FIGURE 5. Behaviors exhibited by Floridobolus penneri. 1. Experiment with 500/50S sediment, $49 \%$ moisture content with five individuals in a 65 gallon terrarium. Two millipedes are in chambers against the glass. Inset: Large chamber with a coiled millipede; molting was later observed in this chamber. 2. Molting individual at the sediment surface. 3. Millipede excavated from $5 \mathrm{~cm}$ below the surface of a $50 \mathrm{Sa} / 50 \mathrm{~S}, 70 \%$ moisture content experiment; the white line indicates the sediment surface.

to elliptical in cross section, but had slightly different dimensions reflecting differences in body size. Narceus americanus burrows had a mean width-toheight ratio of $1.14(0.9-1.4, S D=0.13)$ with a mean circumference of $3.31 \mathrm{~cm}(2.6-4.9, \quad \mathrm{SD}=0.53)$ (Table 2), whereas Floridobolus penneri burrows had a mean width-to-height ratio of 1.06 (0.7-1.6, $\mathrm{SD}=0.18)$ and a mean circumference of $3.8 \mathrm{~cm}$ (2.7-5.2, SD=0.6) (Table 3). The mean width and height of the shafts, tunnels, and chambers varied from 8.2-15.6 $\mathrm{mm}(\overline{\mathrm{x}}=10.6, \mathrm{SD}=1.7)$ and $7.8-12.9$ $\mathrm{mm}(\overline{\mathrm{x}}=9.5, \mathrm{SD}=1.5)$, respectively, for $N$. americanus (Table 2) and 3.95-17.70 mm ( $\bar{x}=12.0$, $\mathrm{SD}=2.5)$ and $5.7-16.7 \mathrm{~mm}(\bar{x}=11.3, \mathrm{SD}=2.24)$, respectively, for $F$. penneri (Table 3 ). Burrow entrances were commonly larger than the burrow. Branching only occurred in $11 \%(n=8)$ of the burrows cast with branching angles ranging from 10$90^{\circ}$ (Tables 2-3). Chambers were observed at the base of many burrows (Figure 5.1). Eight chambers produced by $N$. americanus or $F$. penneri were cast successfully. Chambers of both species typically outlined the general size and shape of the individual that occupied it when in a curled position.

Burrows produced by Narceus americanus and Floridobolus penneri were open structures that were not actively backfilled. Over time, burrow openings were passively filled with loose sediment from the surface. This passive fill process did not always fill the entire burrow; millipedes excavated from beneath closed entrances revealed open shafts and tunnels just below the surface.

Recognizable surficial features were rarely preserved. Vertically-oriented, wedge-shaped indentations were visible at the end of some burrows commonly in association with fecal pellets and burrow chambers (Figure 7.1-2). Fecal pellets were incorporated into the floor of the burrows of both species. The pellets were found in groups of four or five, commonly at an endpoint, and either arranged in a straight line or randomly dispersed (Figure 7.3-5). 


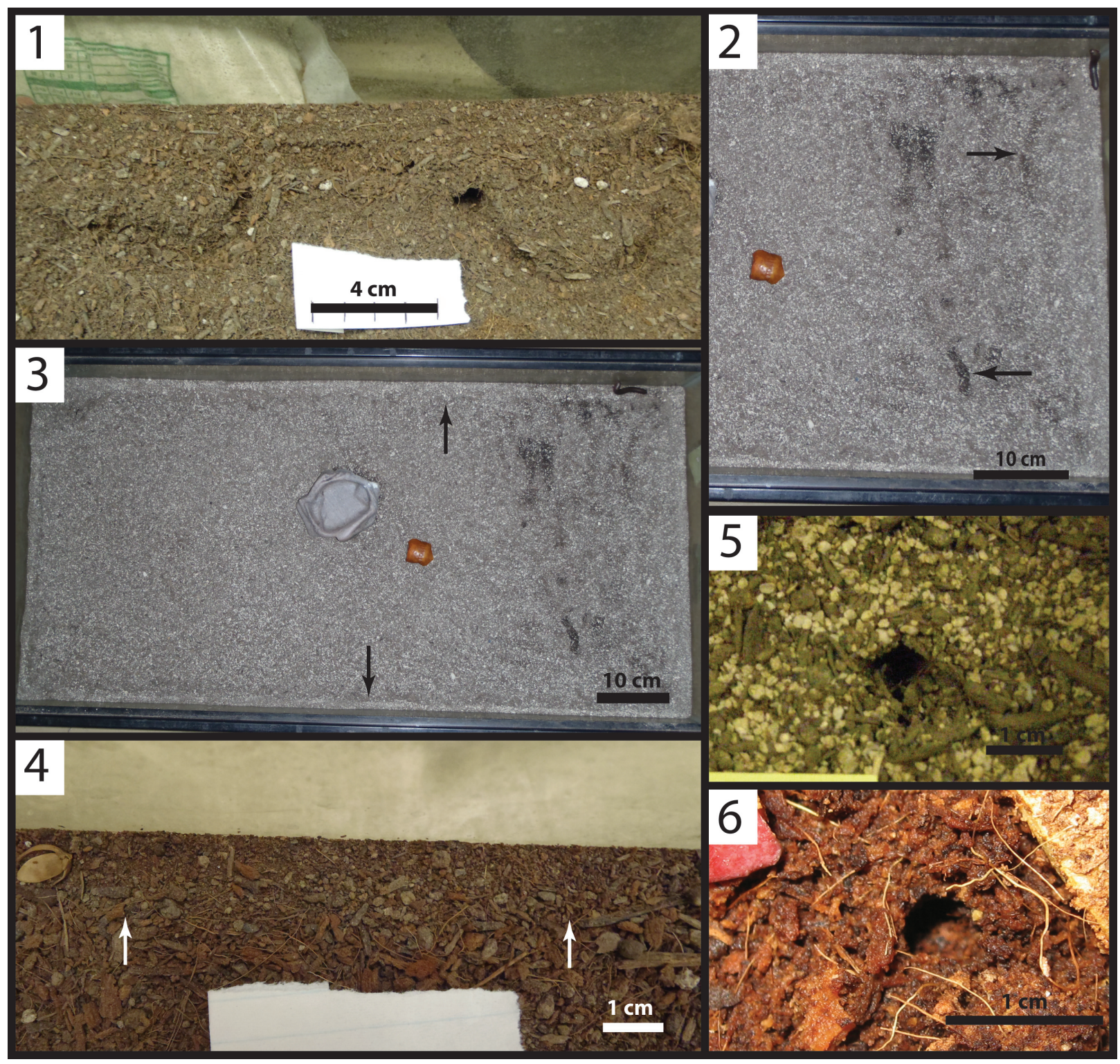

FIGURE 6. Surface traces produced by Narceus americanus and Floridobolus penneri. 1. Surface furrowing and a shallow burrow produced by $N$. americanus in $500 / 50$ S sediment with $35 \%$ moisture content. 2. Surface furrowing (at arrows) produced by $F$. penneri in $25 \mathrm{Sa} / 75 \mathrm{~S}$ sediment with $50 \%$ moisture content. 3 . Trails (at arrows) produced around the edges of a terrarium. 4. Close-up of a trail (at arrows) along the terrarium edge outlined by large grains in $500 / 50 S$ sediment. 5. Burrow entrance produced by F. penneri in $50 \mathrm{Sa} / 50 \mathrm{~S}$ sediment. 6 . Burrow entrance produced by $N$. americanus in $500 / 50$ S sediment.

Vertical Burrows. Burrows consisting of a single opening and a single vertically oriented $\left(80-90^{\circ}, \bar{x}\right.$ $=87.8^{\circ}, \mathrm{SD}=3.6^{\circ} \mathrm{]}$ shaft with a complexity of 2 (Figure 8). Vertical burrows extended $1.90-6.85 \mathrm{~cm}(\bar{x}$ $=3.44, \mathrm{SD}=1.60$ ) below the surface with a total length of $1.90-11.80 \mathrm{~cm}(\bar{x}=4.15, S D=2.70)$ in both species (Tables 2-3). This architecture represented $24 \%(n=7)$ and $21 \%(n=9)$ of burrows produced by Narceus americanus and Floridobolus penneri, respectively.
Narceus americanus produced vertical burrows with widths of $8.15-14.00 \mathrm{~mm}(\bar{x}=11.10$, $\mathrm{SD}=2.20)$, heights of $8.25-12.90 \mathrm{~mm}(\bar{x}=10.20$, $\mathrm{SD}=2.00)$, width-to-height ratios of $0.89-1.30(\bar{x}$ $=1.11, S D=0.14)$, circumferences of $2.60-4.35 \mathrm{~cm}$ $(\overline{\mathrm{x}}=3.50, \mathrm{SD}=0.70)$, and tortuosities of $1.00-1.98(\overline{\mathrm{x}}$ $=1.19, \mathrm{SD}=0.36)$ (Figure 8.1-2) (Table 2). Narceus americanus produced one variant that ended in a horizontally oriented partial spiral (Figure 8.3). The addition of the partial spiral increased both the 

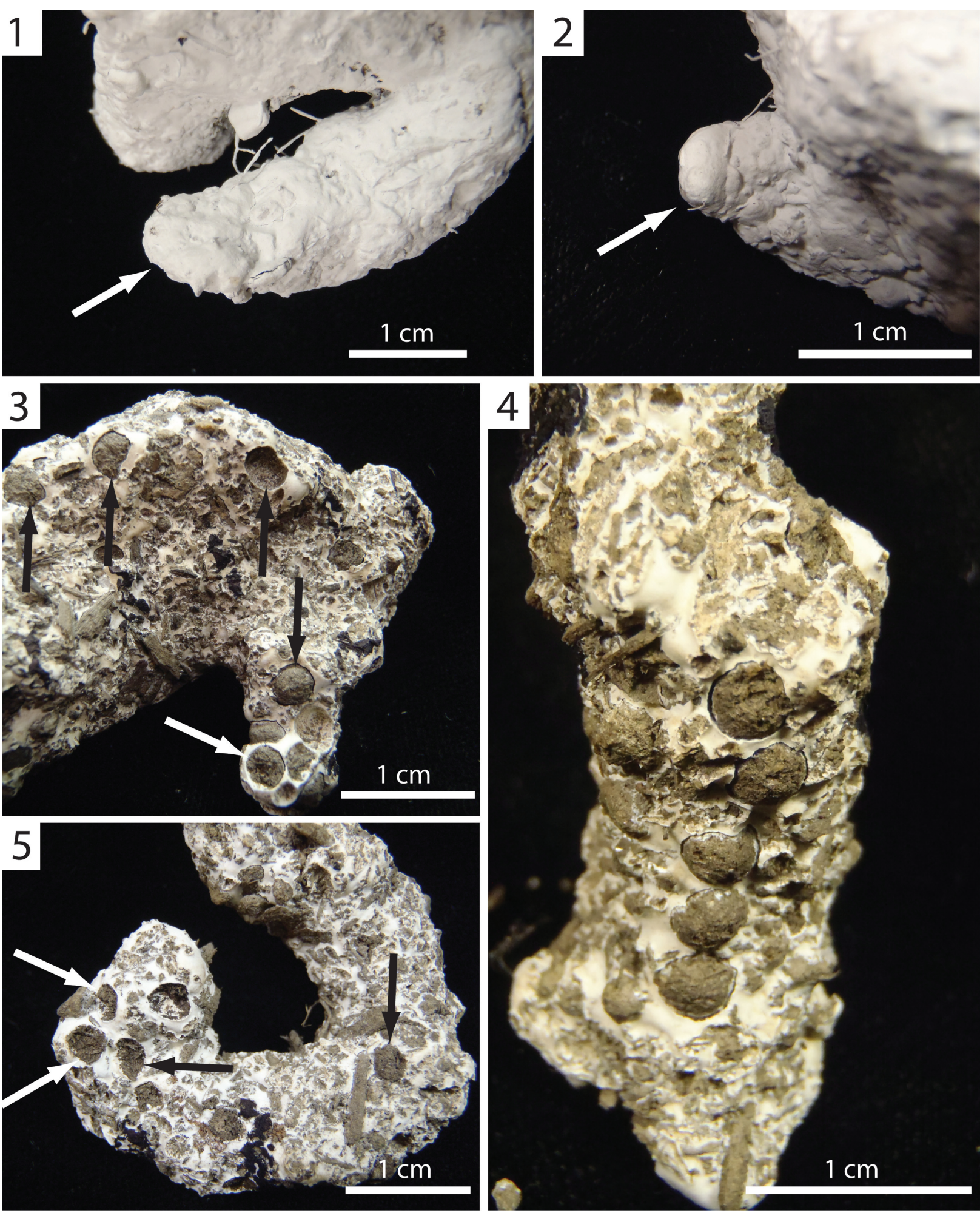

FIGURE 7. Surficial features. 1. Vertically oriented, wedge-shaped indent at the end of NA21F. Photograph taken from below the burrow cast at an oblique angle. 2. Vertically oriented, wedge-shaped indent at the end of NA26A. Photograph taken from below the burrow cast at an oblique angle. 3-5. Fecal pellets in the walls, floors, endpoints, and chambers of three Floridobolus penneri burrows (FP7H, FP7F, FP7G). 

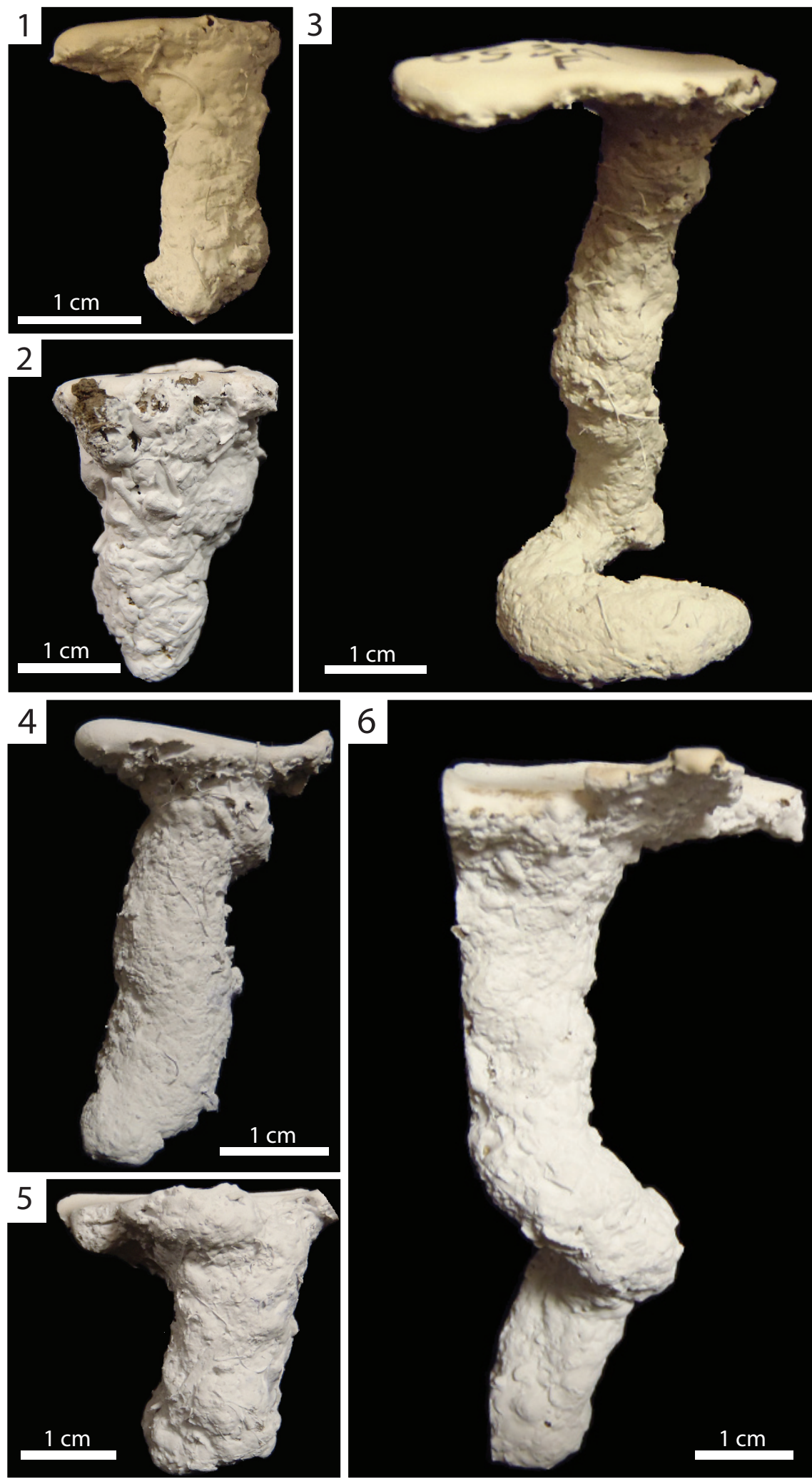

FIGURE 8. Vertical burrows produced by Narceus americanus (1-3) and Floridobolus penneri (4-6). 1, 2. Side views of two simple, vertical burrows (NA30A, NA33A). 3. Oblique view of a vertical burrow with a terminal helical structure (NA30F). 4, 5. Side views of two simple, vertical burrows (FP2A, FP2B). 6. Side view of a vertical burrow with an intermediate helical modification (FP7D). 
length and tortuosity of the burrow. Vertical burrows were produced by $N$. americanus in 65 gallon terraria filled with sediment lacking sand of all moisture contents; $86 \%(n=6)$ occurred in terraria with one individual.

Floridobolus penneri produced vertical burrows with widths of $9.9-16.6 \mathrm{~mm} \quad(\bar{x}=13.10$, $\mathrm{SD}=2.00)$, heights of $9.40-16.70 \mathrm{~mm}(\bar{x}=12.60$, $\mathrm{SD}=2.20)$, width-to-height ratios of $0.74-1.21(\bar{x}$ $=1.02, S D=0.15)$, circumferences of $3.30-5.15 \mathrm{~cm}$ $(\bar{x}=4.13, S D=0.66)$, and tortuosities of $1.00-1.23(\bar{x}$ $=1.06, S D=0.08)($ Figure 8.4-5) (Table 3). A variant was produced with a partial downward spiral in the middle of the burrow (Figure 8.6). Vertical burrows were produced by $F$. penneri in all terraria sizes and all sediment compositions with high moisture content; $78 \%(n=7)$ occurred in terraria with multiple individuals.

Subvertical Burrows. Burrows consisting of a single opening leading to a single tunnel having a mean slope of less than $80^{\circ}\left(20-78^{\circ}, \bar{x}=60.9^{\circ}\right.$, $\mathrm{SD}=14.5^{\circ}$ ) that may possess a chamber or branch (Figures 9, 10). Subvertical burrows of both species were $1.50-13.40 \mathrm{~cm}(\overline{\mathrm{x}}=5.30, \mathrm{SD}=2.80)$ deep and $1.70-18.60 \mathrm{~cm}$ long $(\bar{x}=7.00, S D=3.90)$ (Tables 2-3). Subvertical burrows represented $45 \%(n=13)$ and $52 \%(n=22)$ of the burrows produced by Narceus americanus and Floridobolus penneri, respectively.

The shafts, tunnels, and chambers of subvertical burrows produced by Narceus americanus were $8.80-15.50 \mathrm{~mm}(\bar{x}=10.80, S D=1.80)$ wide and $7.90-12.20 \mathrm{~mm}(\overline{\mathrm{x}}=9.40, \mathrm{SD}=1.40)$ high with width-to-height ratios of $0.96-1.40 \quad(\bar{x}=1.17$, $S D=0.15)$, circumferences of $2.65-4.90 \mathrm{~cm}(\bar{x}$ $=3.30, \mathrm{SD}=0.58)$, and tortuosities of $1.00-1.70(\bar{x}$ $=1.14, \mathrm{SD}=0.18$ ) (Figure 9.1-2) (Table 2). One subvertical burrow had a branch (Figure 9.3) and four had chambers (Figure 9.4-5). Subvertical burrows of $N$. americanus were primarily $(92 \%, \mathrm{n}=12)$ produced in the 500/50S sediment compositions under all moisture conditions and terrarium sizes; most $(69 \%, n=9)$ were produced in terraria with more than one individual.

Subvertical burrows produced by Floridobolus penneri were slightly larger with widths of 3.95$16.80 \mathrm{~mm}(\bar{x}=11.80, S D=2.60)$, heights of 5.70 $16.50 \mathrm{~mm}(\bar{x}=11.30, S D=2.30)$, width-to-height ratios of $0.70-1.38(\bar{x}=1.05, S D=0.17)$, circumferences of $2.65-5.15 \mathrm{~cm}(\bar{x}=3.80, S D=0.61)$, and tortuosities of $1.00-1.60(\bar{x}=1.17, S D=0.16)$ (Figure 10.1-2) (Table 3). Two had a branch and one had a chamber (Figure 10.3-4). One large subver- tical burrow had a large chamber with a branch (Figure 10.5). Subvertical burrows produced by $F$. penneri occurred in all terrarium sizes and sediment compositions with high moisture content; $64 \%(n=14)$ were produced in terraria with multiple individuals.

Helical Burrows. Burrows with a single surface opening leading to a spiraling tunnel with a mean tortuosity of $3.8(2.30-5.60, S D=1.70)$ that may possess a branch or a chamber (Figure 11). Helical burrows of both species were $2.5-3.4 \mathrm{~cm}(\bar{x}=2.92$, $\mathrm{SD}=0.30)$ deep and $6.80-11.10 \mathrm{~cm}(\bar{x}=9.30$, $S D=1.90$ ) long (Tables $2-3$ ). The helical nature of these burrows resulted in a lower mean slope than other burrow architectures $\left(\bar{x}=47.0^{\circ}, 25-75^{\circ}\right.$, $\mathrm{SD}=22.8^{\circ}$ ). Helical burrows accounted for $17 \%$ $(n=5)$ and $5 \%(n=2)$ of the burrows produced by Narceus americanus and Floridobolus penneri, respectively.

Narceus americanus produced helical burrows with widths of $8.40-11.64 \mathrm{~mm}(\bar{x}=9.70$, $S D=1.30)$, heights of $7.80-9.80 \mathrm{~mm}(\bar{x}=8.70$, $\mathrm{SD}=0.90)$, width-to-height ratios of $0.98-1.30(\bar{x}$ $=1.12, S D=0.12)$, and circumferences of 2.70-3.40 $\mathrm{cm}(\bar{x}=3.04, S D=0.29)$ (Figure 11.1) (Table 2). One helical burrow possessed a branch (Figure 11.2) and another possessed a chamber (Figure 11.3). Helical burrows of $N$. americanus were produced in sediments without sand, under all moisture conditions and terrarium sizes, with both multiple and single individuals.

Floridobolus penneri produced only two helical burrows, one simple and one with two branches (Figure 11.4-5). The helical burrows had widths of $9.80-11.60 \mathrm{~mm}(\bar{x}=10.70, S D=1.25)$, heights of 6.10-9.90 mm ( $\bar{x}=8.00, S D=2.80)$, width-to-height ratios of $1.17-1.60(\bar{x}=1.40, S D=0.32)$, and circumferences of $2.70-3.60 \mathrm{~mm}(\overline{\mathrm{x}}=3.10, \mathrm{SD}=0.70)$ (Table 3 ). Helical burrows were produced by $F$. penneri in sediments with high moisture content in trials with single individuals.

O-shaped Burrows. Burrows consisting of one or two surface openings leading to a single horizontally or vertically oriented tunnel that loops and reconnects with itself which may possess a chamber or branches (Figure 12). The O-shaped portions occurred either at the end of an elongate shaft (Type 1) or as horizontally oriented structures just below the surface (Type 2). The O-shaped burrows were $1.10-7.70 \mathrm{~cm}(\bar{x}=4.00, S D=2.50)$ deep and $10.00-17.50 \mathrm{~cm}(\bar{x}=12.30, S D=2.60)$ long. This architecture accounted for $14 \%(n=4)$ and $7 \%$ 

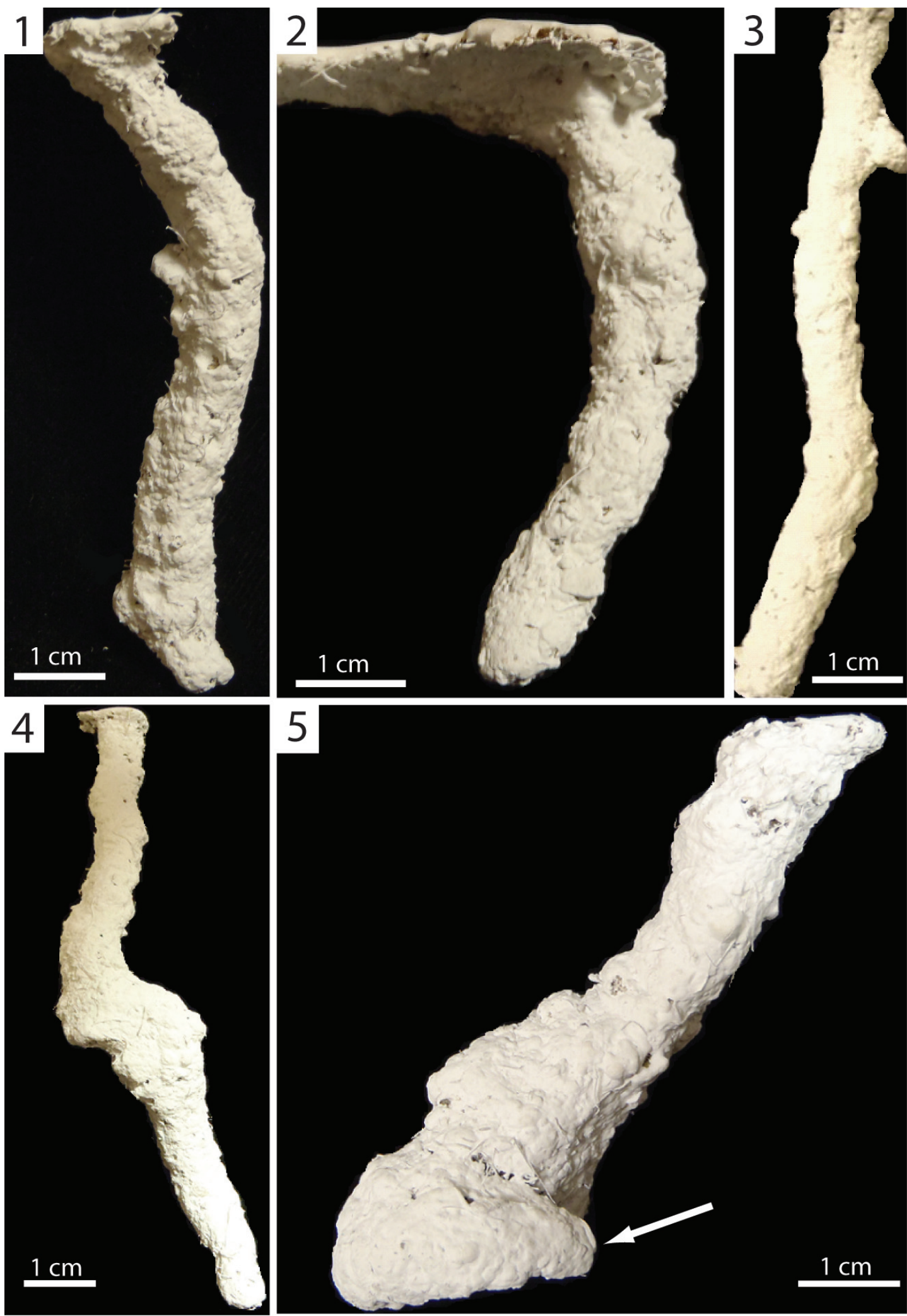

FIGURE 9. Subvertical burrows produced by Narceus americanus. 1. Side view of a simple, subvertical burrow (NA21C). 2. Side view of a simple, subvertical burrow (NA30D). 3. Front view of a subvertical burrow with a small branch near the entrance (NA21D). 4. Front view of a subvertical burrow with a small intermediate chamber (NA21E). 5. Side view of a subvertical burrow with a terminal chamber (NA26A) and a wedge-shaped indent (at arrow). 

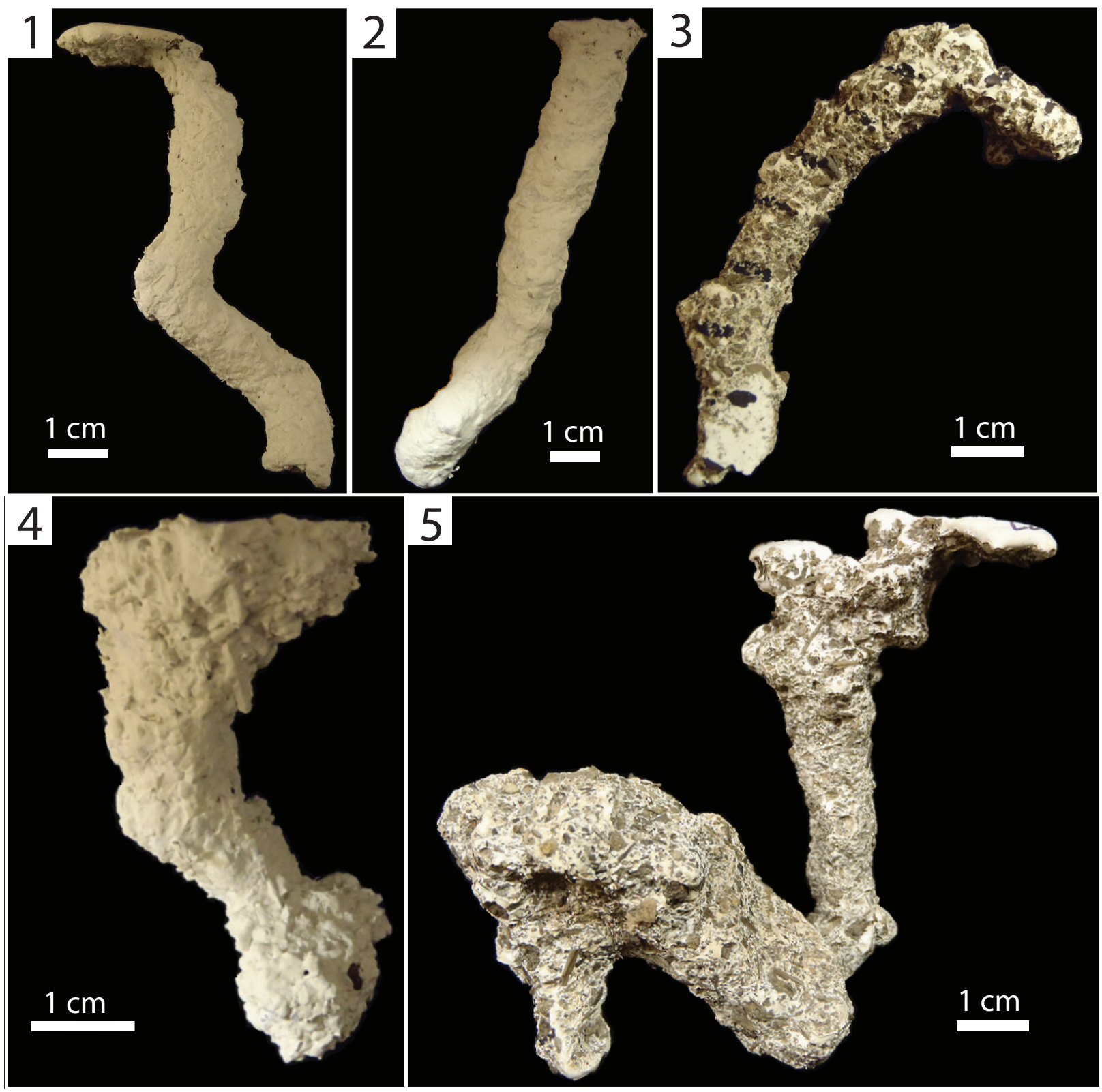

FIGURE 10. Subvertical burrows produced by Floridobolus penneri. 1. Front view of a simple, sinuous, subvertical burrow (FP2F). 2. Side view of a simple, subvertical burrow (FP2E). 3. Side view of a subvertical burrow with a small branch near the entrance (FP7F). 4. Side view of a subvertical burrow with a terminal chamber (FP5E). 5. Side view of a subvertical burrow with a large chamber and small branch (FP7H).

$(\mathrm{n}=3)$ of burrows produced by Narceus americanus and Floridobolus penneri, respectively.

O-shaped burrows of Narceus americanus had widths of $10.10-10.90 \mathrm{~mm} \quad(\bar{x}=10.50$, $\mathrm{SD}=0.34)$, heights of $8.20-10.40 \mathrm{~mm}(\bar{x}=9.50$, $\mathrm{SD}=1.00$ ), width-to-height ratios of $1.00-1.30 \overline{\mathrm{x}}=$ $1.10, S D=0.13)$, circumferences of $3.10-3.50 \mathrm{~cm}(\bar{x}$ $=3.30, S D=0.18)$, and tortuosities of 1.80-99.00 ( $\bar{x}$ $=45.70, S D=40.10)$ (Figure 12.1-4) (Table 2). The $\mathrm{O}$-shaped burrows produced by $\mathrm{N}$. americanus were simple with the exception of one that possessed two surface openings (Figure 12.3). Narceus americanus constructed Type 1 O-shaped burrows in 50O/50S and 50Sa/50S sediment, under all moisture conditions, terrarium sizes, and in both individual and group trials. Type 2 Oshaped burrows were only produced in trials with carbonate sand.

Floridobolus penneri produced O-shaped burrows with widths of $10.10-17.70 \mathrm{~mm}(\bar{x}=12.90$, 

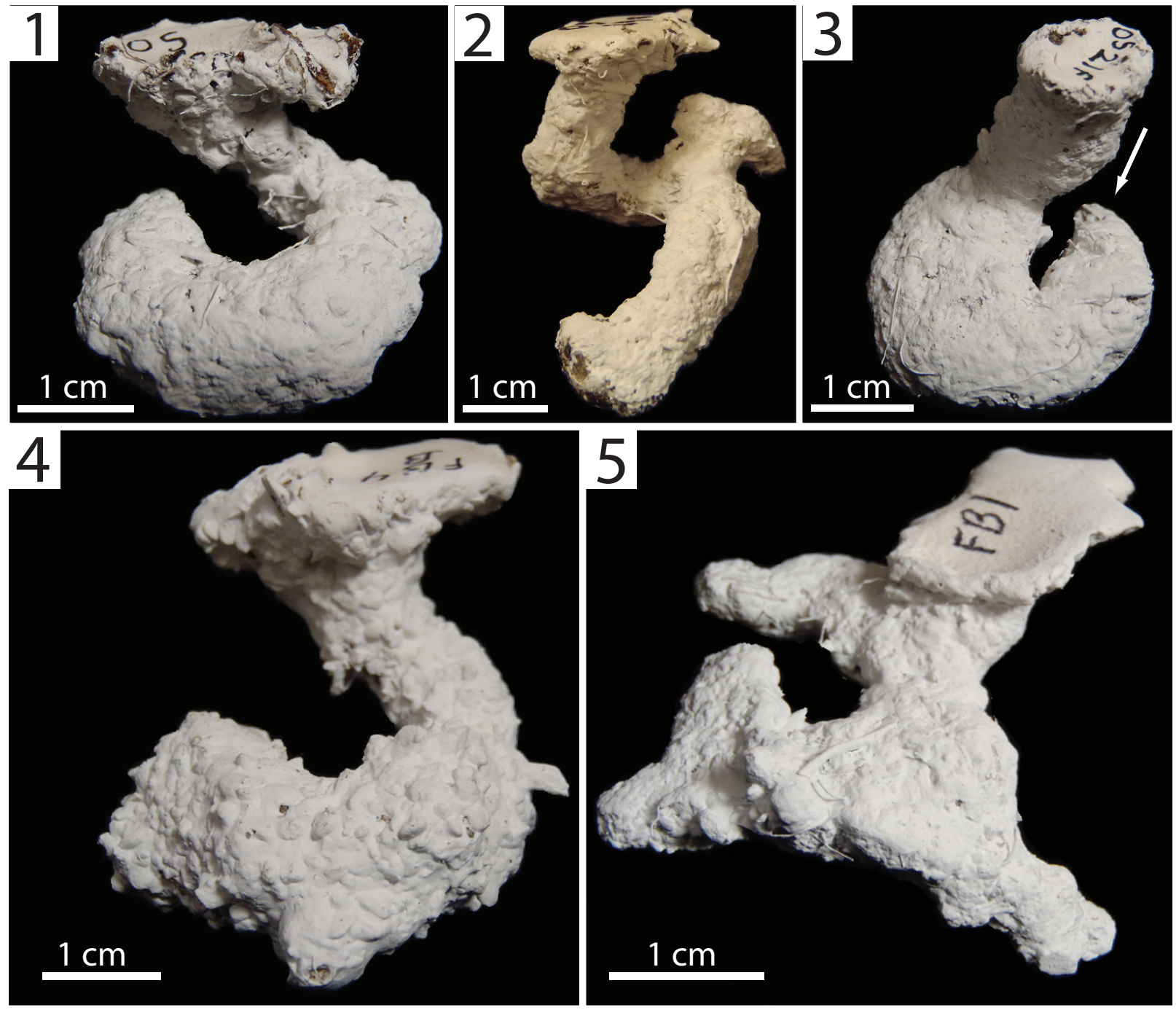

FIGURE 11. Helical burrows produced by Narceus americanus (1-3) and Floridobolus penneri (4-5). 1. Side view of a simple, helical burrow (NA25B). 2. Side view of a helical burrow with a small branch (NA29B). 3. Oblique view of a helical burrow with a chamber in the midsection of the burrow (NA21F) bearing a wedge-shaped indent (at arrow). 4. Side view of a simple, helical burrow (FP4B). 5. Oblique view of a helical burrow with two small branches (FP1).

$\mathrm{SD}=4.20)$, heights of $8.30-12.20 \mathrm{~mm}(\overline{\mathrm{x}}=10.80$, $\mathrm{SD}=2.20)$, width-to-height ratios of $0.90-1.46(\bar{x}$ $=1.16, \mathrm{SD}=0.20)$, circumferences of $3.00-4.40 \mathrm{~cm}$ $(\bar{x}=3.50, S D=0.50)$, and tortuosities of $1.40-53.00$ $(\bar{x}=24.00, S D=26.40)$ (Figure 12.5-7) (Table 3). The O-shaped burrows produced by $F$. penneri possessed extra elements such as one or two horizontal branches (Figure 12.5, 12.7) and chambers (Figure 12.6). O-shaped burrows were produced by $F$. penneri in sandy soil sediments with high moisture content in trials with multiple individuals using 30 gallon terraria.

J-shaped Burrows. Burrows consisting of a single surface opening leading to a single vertical to subvertical shaft that curves upward at its base to cre- ate a J-shape (Figure 13.1-2). J-shaped burrows were produced only by Floridobolus penneri, accounting for $14 \%(n=6)$ of its burrows. J-shaped burrows were $2.90-8.40 \mathrm{~cm}(\overline{\mathrm{x}}=6.60, \mathrm{SD}=2.10)$ deep and $7.50-14.70 \mathrm{~cm}(\bar{x}=11.60, S D=2.40)$ long. The shafts had widths of $9.40-12.00 \mathrm{~mm}(\bar{x}=10.70$, $\mathrm{SD}=0.94)$, heights of $9.50-13.00 \mathrm{~mm}(\bar{x}=11.40$, $\mathrm{SD}=1.20)$, width-to-height ratios of $0.85-1.00(\bar{x}$ $=0.95, S D=0.06)$, and circumferences of 3.10-3.90 $\mathrm{cm}(\overline{\mathrm{x}}=3.60, \mathrm{SD}=0.32)$ (Table 3). One variant was produced with a branch near the entrance of the burrow (Figure 13.3). J-shaped burrows were produced in sediments without carbonate sand with high moisture content and in trials with multiple individuals. 

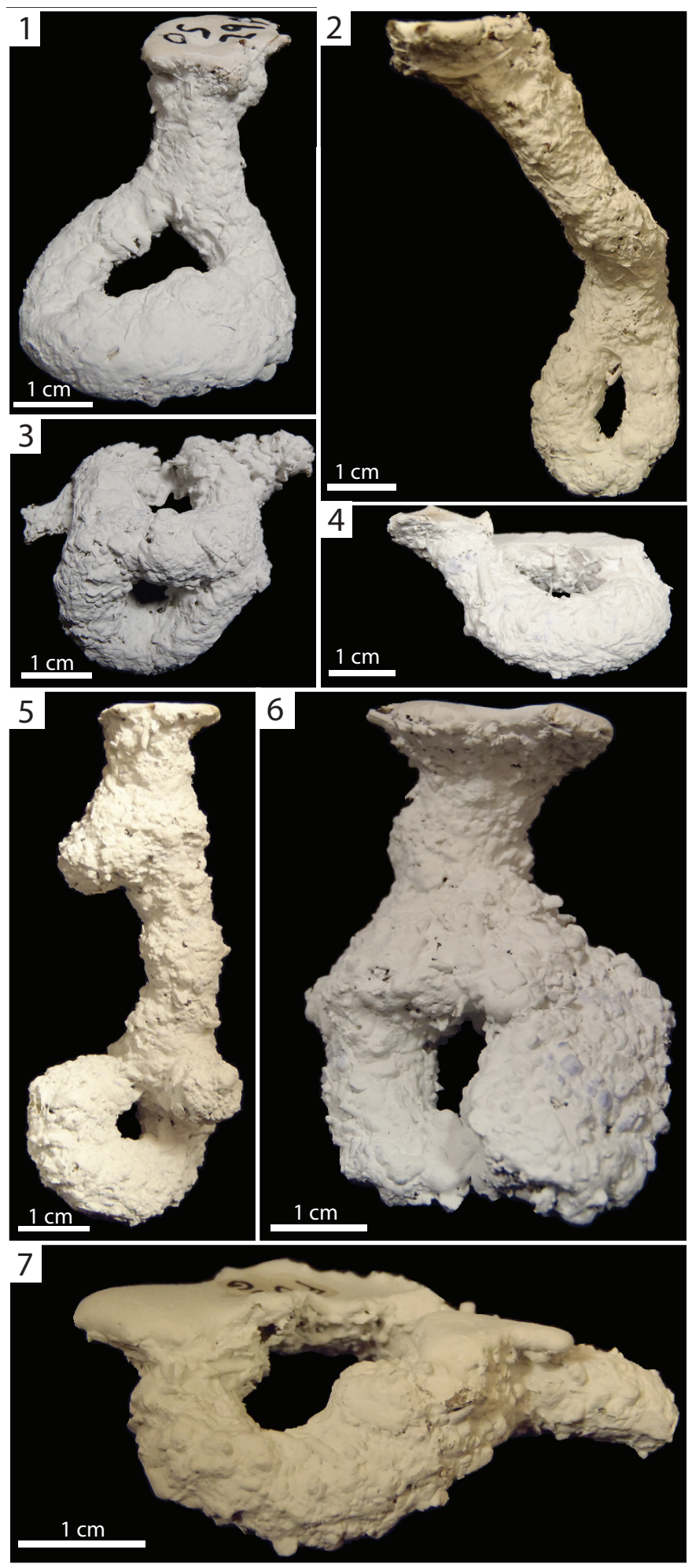

FIGURE 12. O-shaped burrows produced by Narceus americanus (1-4) and Floridobolus penneri (5-7). 1. Front oblique view of a horizontally oriented, Type 1 O-shaped burrow (NA29A). 2. Side view of a vertically oriented, Type 1 O-shaped burrow (NA26B). 3. Oblique view of the underside of a horizontally oriented, Type 2 O-shaped burrow with two surface openings (NA31A). 4. Side view of a Type 2 O-shaped burrow (NA31B). 5. Side view of a vertically oriented, Type 1 O-shaped burrow with two short branches (FP5F). 6. Front view of a vertically oriented, Type 1 Oshaped burrow with a chamber on one side of the O-shaped tunnel (FP6B). 7. Oblique side view of a Type 2 Oshaped burrow with a branch (FP5G). 

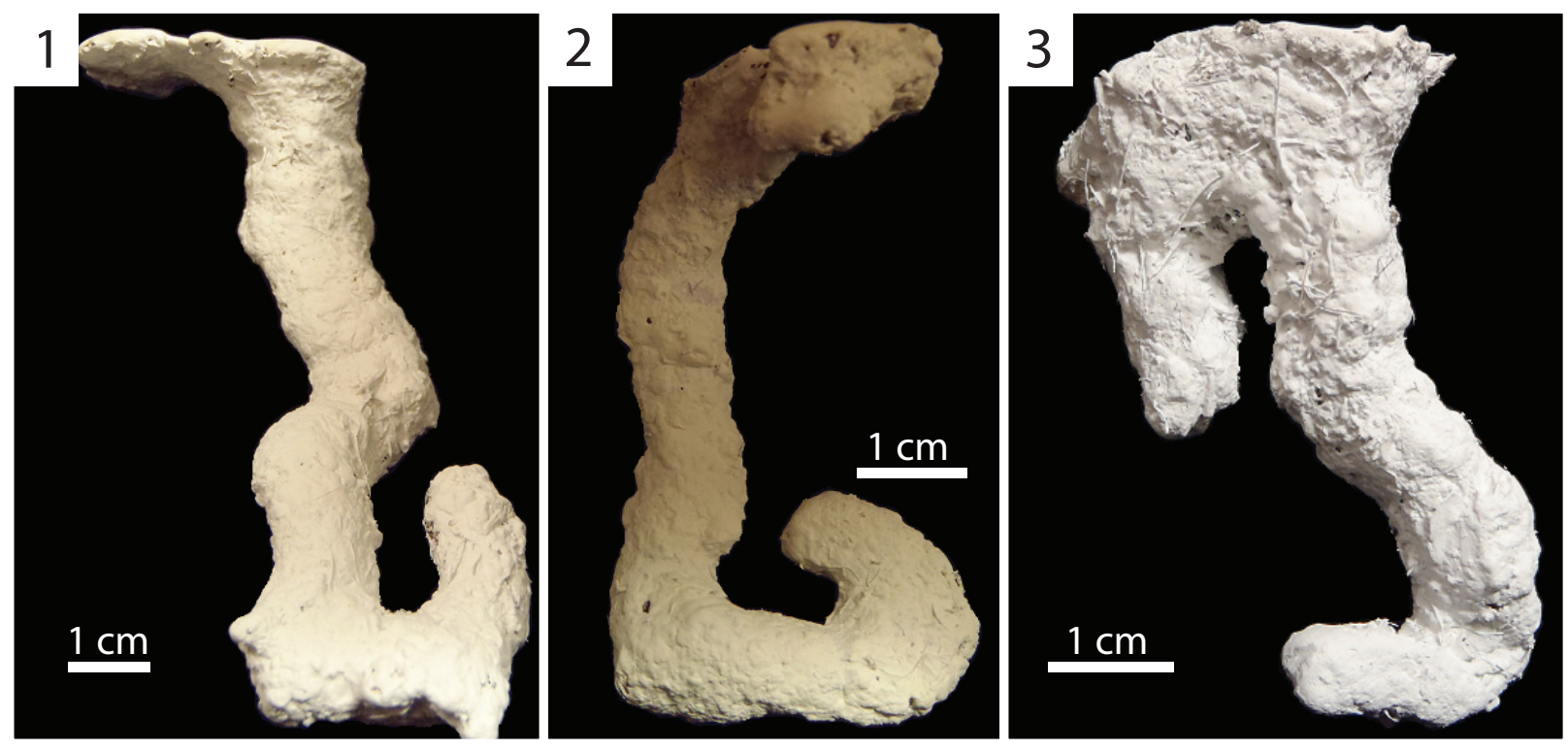

FIGURE 13. J-shaped burrows produced by Floridobolus penneri. 1. Front view of a simple, J-shaped burrow (FP2J). 2. Front view of a simple, J-shaped burrow (FP2I). 3. J-shaped burrow with a branch near the entrance (FP2H).

\section{Environmental Effects}

Environmental variations affected aspects of the millipede's behavior, burrow preservation, and burrow morphology. The variations which appeared to have the greatest effect were the presence of multiple individuals, low moisture content, and high sand content. Floridobolus penneri, for example, was a more proficient burrower in sandy sediments producing 11 preserved structures with seven different architectures. In contrast, Narceus americanus produced only three structures in sandy sediment with three architectures.

Burrow preservation was lowered by the passive infilling of burrow openings. In trials with multiple individuals the upper portions of the millipede burrows became passively filled as continued animal surface activity caused grains to collapse into the openings. Fewer successful burrow casts were also produced in experiments with carbonate sand in which the individuals remained in their burrows longer than the anticipated duration of the trial. The lack of consistent use and maintenance of the entrance resulted in gravitational collapse of sand grains into the burrow, filling the opening. Low moisture conditions were also found to decrease the potential for burrow entrances to remain open for the duration of the experiment. In nine trials with Narceus americanus under $30-40 \%$ moisture conditions, only 14 burrows were successfully cast (06 burrows per trial). In contrast, under $\geq 50 \%$ moisture conditions, nine burrows were cast in only two trials (3-6 burrows per trial).
Moisture and sand content affected the maximum recorded depth of the burrows. Casts of burrows constructed by Narceus americanus under drier conditions reached a maximum depth of $13.40 \mathrm{~cm}(\bar{x}=5.40 \mathrm{SD}=3.60)$ but a maximum depth of only $6.60 \mathrm{~cm}(\bar{x}=3.80 \mathrm{SD}=1.80)$ under wetter conditions. The maximum depth of burrows produced by both species also decreased with higher concentrations of carbonate sand. Casts of burrows produced by $N$. americanus reached a mean maximum depth of $4.1 \mathrm{~cm}$ in $25 \mathrm{Sa} / 75 \mathrm{~S}$ sediment, but only $2.20 \mathrm{~cm}(1.90-2.50, \mathrm{SD}=0.40)$ in $50 \mathrm{Sa} /$ $50 S$ sediment. Similarly, burrow casts of Floridobolus penneri had a mean maximum depth of $4.1 \mathrm{~cm}(1.10-7.70, \mathrm{SD}=2.30)$ in $25 \mathrm{Sa} / 75 \mathrm{~S}$ sediment, but only $3.1 \mathrm{~cm}(1.80-4.40, \mathrm{SD}=1.10)$ in $50 \mathrm{Sa} / 50 \mathrm{~S}$ sediment. Terrarium size and the associated sediment depth appeared to have no effect on burrow depth or architecture. While individuals were observed to reach depths of $\sim 33 \mathrm{~cm}$ in some trials, this was not the bottom of the enclosure. When retrieved, no individuals were found at the bottom of the terraria.

\section{Analysis of Burrow Architecture}

Burrows of Narceus americanus. Burrows produced by Narceus americanus were found to range from dissimilar (0.5-0.3) to identical (1.0) (Figures 14, 15; Appendices 5, 6). The mean similarity for all burrows produced by $N$. americanus was 0.8 , indicating a highly similar suite of burrows based on the 10 quantitative properties (Figure 14, 15). Of the 29 burrow casts, all but one O-shaped burrow 


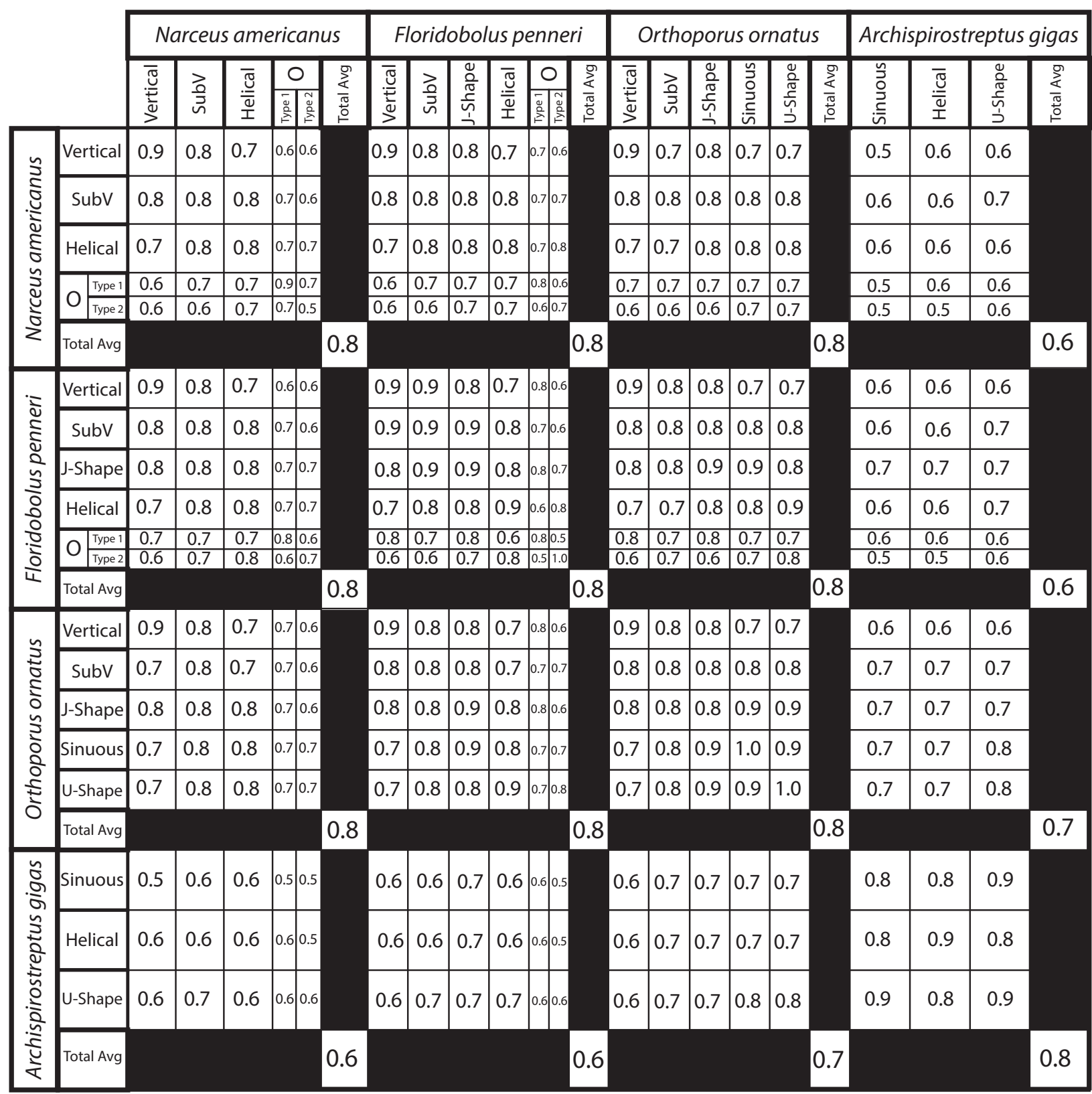

FIGURE 14. Summary of Bray Curtis similarity test results for the burrows of the four millipede species. Values are arithmetic means of the similarity values produced when burrows of similar or different architectures are compared. Total Avg columns represent the mean value of similarity when all burrows produced by one species are compared to those produced by itself or another species. Values of $0.9-0.8$ indicate high similarity, $0.7-0.6$ indicate moderate similarity, and values of 0.5 or less indicate dissimilarity. Values of 1.0 indicate that there was only one burrow produced of that architecture by that species.

(NA31B) were found to be at least moderately similar (>0.65) (Figure 15). Higher levels of similarity $(>0.75)$ were primarily controlled by primary architecture (Figure 15).

Similarity values within each burrow architecture had a mean of 0.9 for vertical burrows, 0.8 for subvertical burrows, 0.8 for helical burrows, and 0.7 for O-shaped burrows (Figure 14; Appendices
$5,6)$. Moderate levels of similarity $(0.7-0.6)$ within burrow architectures were primarily restricted to only a few burrows (Appendices 5, 6). Of all subvertical burrows $(n=13)$, for example, only $15 \%$ $(n=2)$ were responsible for all occurrences of moderate similarity (0.7). Among vertical burrows $(n=7)$, only one (NA30F) produced all results of moderate similarity (0.7). Similarities between heli- 


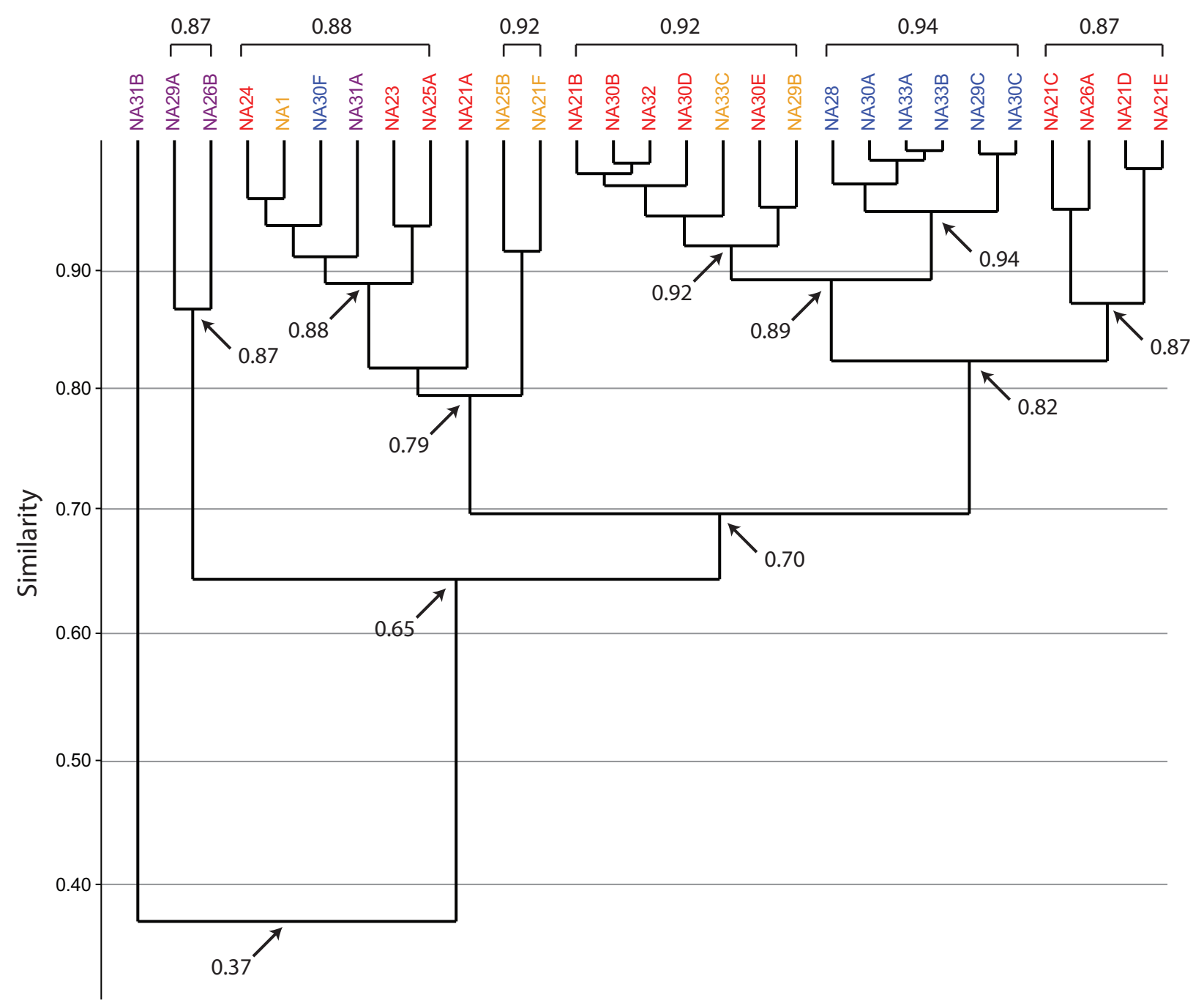

FIGURE 15. Bray Curtis cluster diagram comparing the 29 burrow casts produced by Narceus americanus. Burrow cast ID numbers are color coded by architecture: red = subvertical burrow; blue = vertical burrow; orange = helical burrow; purple $=0$-shaped burrow. Branch points near the top of the diagram indicate higher levels of similarity. Key branch points are marked with their similarity value (at arrows). Values $>0.80$ indicate high similarity, $0.79-0.60$ indicate moderate similarity, and values of 0.59 or less indicate dissimilarity. Groups of burrow casts found to be most similar are marked with brackets and their level of similarity.

cal burrows $(n=5)$ were less well defined. Only one helical burrow (NA1) was highly similar to all other helical burrows while the rest were highly similar $(0.9-0.8)$ to only two and moderately similar (0.7) to the remaining two burrows (Figure 15; Appendices 5,6$)$. O-shaped burrows were the least similar to each other. Within the O-shaped burrows $(n=4)$, the comparison of NA26A and NA26B produced the only result of high similarity (0.9). All other comparisons resulted in moderate similarity $(0.7-0.6)$ with the exception of the comparison of NA31A and NA31B which produced the only result of dissimilarity (0.5) among the burrows of Narceus americanus.
When different architectures were compared there was a tendency toward decreased similarity as the mean angle of the burrow system decreased and tortuosity increased (Figures 14, 15; Appendix 5). Vertical burrows, for example, had a mean similarity of 0.8 with subvertical burrows, 0.7 with helical burrows, and 0.6 with $\mathrm{O}$-shaped burrows. A few burrows that were qualitatively characterized as having different architectures were found to be highly similar, indicating that other properties were also contributing to similarity (Figures 14, 15; Appendix 5). For example, two helical burrows (NA33C, NA29B) were highly similar to all vertical burrows, and most subvertical burrows (Figure 15). 


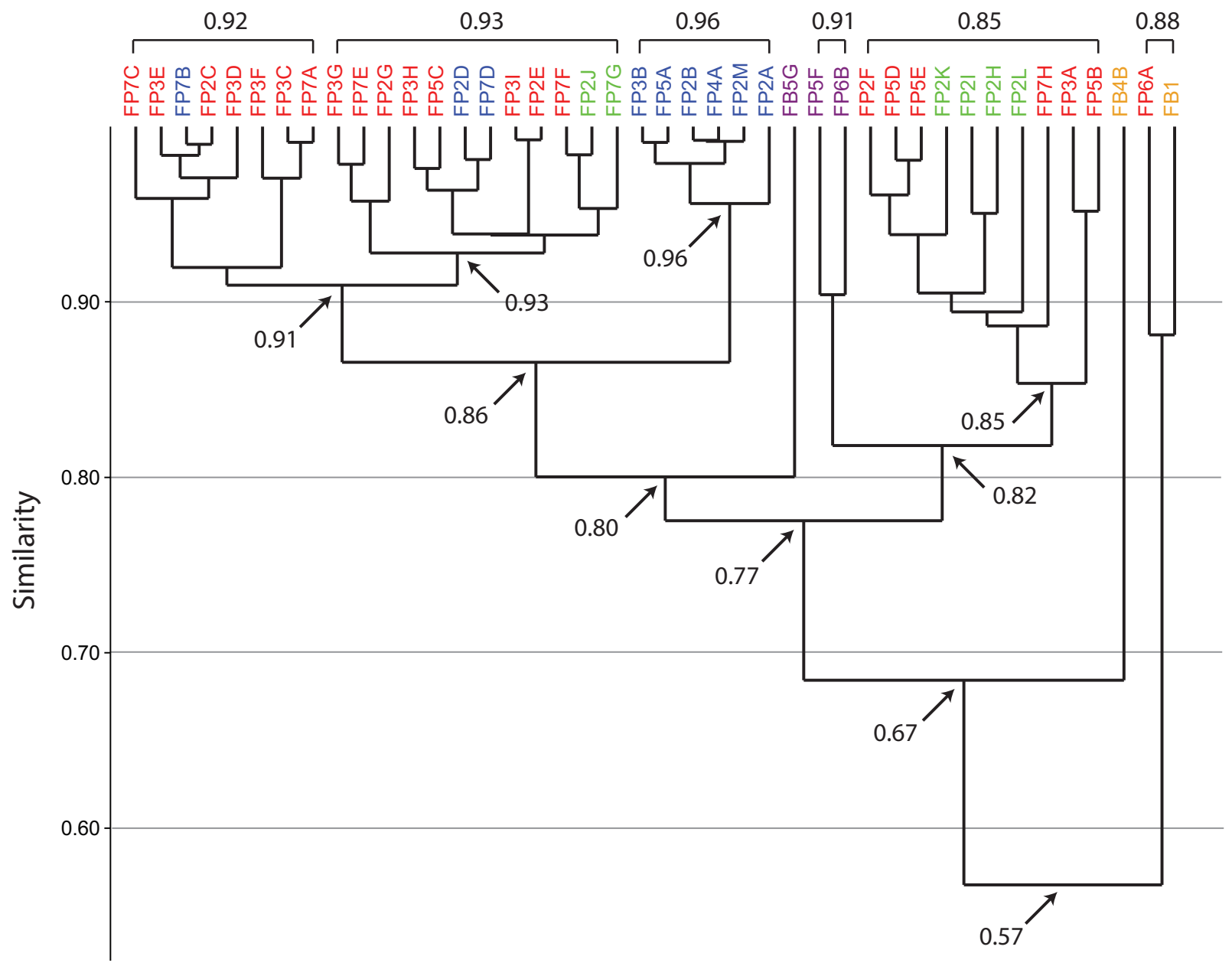

FIGURE 16. Bray Curtis cluster diagram comparing the 42 burrow casts produced by Floridobolus penneri. Burrow cast ID numbers are color coded by architecture: red = subvertical burrow; blue = vertical burrow; orange = helical burrow; purple = O-shaped burrow; green $=\mathrm{J}$-shaped burrow. Branch points near the top of the diagram indicate higher levels of similarity. Key branch points are marked with their similarity value (at arrows). Values $>0.80$ indicate high similarity, $0.79-0.60$ indicate moderate similarity, and values of 0.59 or less indicate dissimilarity. Groups of burrow casts found to be most similar are marked with brackets and their level of similarity.

Burrows categorized by the Bray Curtis analysis to be identical $(n=13)$ were limited to those in the same architecture with only two exceptions (NA33C-helical burrow, NA30D-subvertical burrow) (Appendices 5, 6).

Burrows of Floridobolus penneri. Burrows produced by Floridobolus penneri were found to range from dissimilar (0.5) to identical (1.0) (Figures 14, 16; Appendices 5,7). The mean similarity of all burrows produced by $F$. penneri was 0.8 indicating that the suite was highly similar (Figure 14). These similarities were highest within burrow architectures (Figure 14; Appendix 7). Of the 42 burrow casts, all but two burrows were found to be at least moderately similar (>0.65) (Figure 16). Higher levels of similarity $(>0.75)$ resulted from similar primary architectures (Figure 16).
Vertical, subvertical, J-shaped, and helical burrows all had mean similarity values of 0.9 indicating very high similarity (Figure 14; Appendix 7). Only O-shaped burrows had a mean similarity $(0.6)$ that indicated moderate similarity (Figure 14). Within the same burrow architectures, only two burrows (FP6A-subvertical, FP5G-O-shaped) accounted for the majority of moderately similar $(0.7-0.6)$ and dissimilar (0.5) results (Figure 16; Appendix 7).

When burrows of different architectures were compared, a trend similar to that seen in Narceus americanus burrows was found; as the mean angle of the burrow system decreased and tortuosity increased, similarity decreased. Vertical burrows had a mean similarity of 0.9 with subvertical burrows, 0.8 with $\mathrm{J}$-shaped burrows, 0.7 with helical 


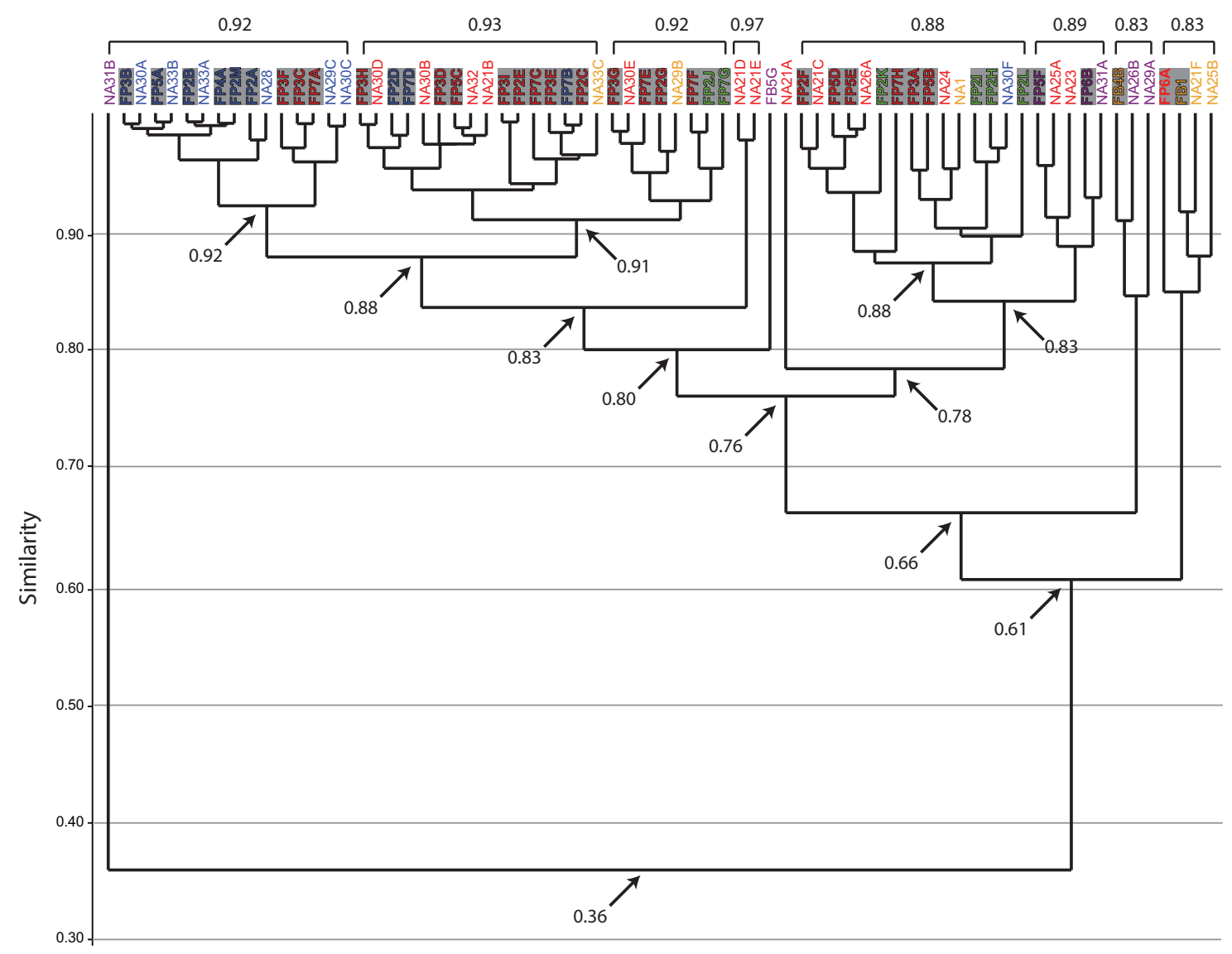

FIGURE 17. Bray Curtis cluster diagram comparing the 71 burrow casts produced by Narceus americanus (NA) and Floridobolus penneri (FP; shaded gray). Burrow cast ID numbers are color coded by architecture: red = subvertical burrow; blue = vertical burrow; orange = helical burrow; purple = O-shaped burrow; green = J-shaped burrow. Branch points near the top of the diagram indicate higher levels of similarity. Key branch points are marked with their similarity value (at arrows). Values $>0.80$ indicate high similarity, $0.79-0.60$ indicate moderate similarity, and values of 0.59 or less indicate dissimilarity. Groups of burrow casts found to be most similar are marked with brackets and their level of similarity.

burrows, and 0.7 with $\mathrm{O}$-shaped burrows (Figure 14, 16; Appendix 5). The occurrence of identical burrows between different architectures $(n=19)$ was limited to comparisons between vertical, subvertical, and J-shaped burrows (Appendix 5).

\section{Burrows of Narceus americanus and} Floridobolus penneri. The burrows of both species had an overall high mean similarity value (0.8) (Figures 14, 17; Appendices 5, 8). Of the 71 burrows analyzed, only one (NA31B) was found to be dissimilar and only seven had a similarity of $<0.76$ (Figure 17). Sixty-four burrows of both Narceus americanus and Floridobolus penneri, therefore, were found to be moderately to highly similar $(>0.76)$ (Figure 17). Burrows of both species occurred in clusters at the highest levels of similarity $(>0.90)$, most, but not all, having similar architectures (Figure 17).
Comparisons of each of the four common architectures resulted in similarity values that were close to those of the individual species (Figures 14, 17; Appendices 5, 8). The mean similarity of subvertical and vertical burrows produced by both species was 0.9. Within the subvertical architecture, only three (NA21A, NA25A, FP6A) of 35 burrows produced most $(86 \%)$ of the moderately similar or dissimilar values (Appendix 8). Of the 16 vertical burrows, only a single burrow with a helical component (NA30F) produced all moderate similarity values (Appendix 8). Helical burrows of both species were also found to be highly similar $(0.8)$ with only one instance of moderate similarity between two (FP4B, NA29B) out of seven burrows (Appendix 8). The O-shaped burrows of each species had a moderate (0.7) level of similarity. When split into Type 1 and Type 2 structures, however, Type 1 O- 


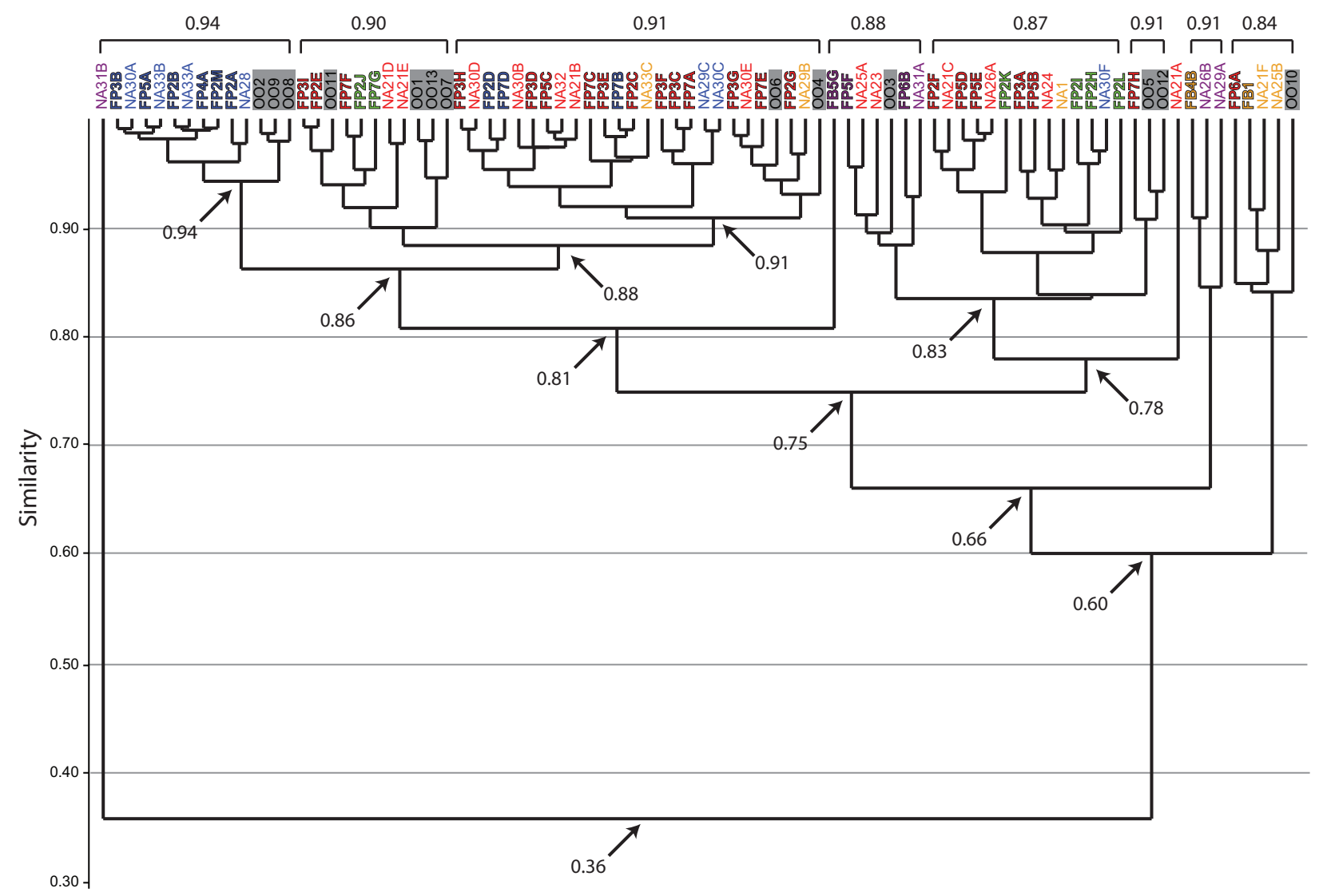

FIGURE 18. Bray Curtis cluster diagram comparing the 71 burrow casts produced by Narceus americanus (NA) and Floridobolus penneri (FP) with 13 burrow casts produced by Orthoporus ornatus (OO; shaded gray) from Hembree (2009). Burrow cast ID numbers are color coded by architecture: red = subvertical burrow; blue = vertical burrow; orange $=$ helical burrow; purple $=\mathrm{O}$-shaped burrow; green $=\mathrm{J}$-shaped burrow. Branch points near the top of the diagram indicate higher levels of similarity. Key branch points are marked with their similarity value (at arrows). Values $>$ 0.80 indicate high similarity, $0.79-0.60$ indicate moderate similarity, and values of 0.59 or less indicate dissimilarity. Groups of burrow casts found to be most similar are marked with brackets and their level of similarity.

shaped burrows had a high mean similarity value (0.8) whereas Type 2 O-shaped burrows had a moderate mean similarity value (0.6) (Appendix 8). The lower level of similarity within Type 2 burrows is due to the simple Type 2 burrow of Narceus americanus (NA31B) and was likely related to that burrow's high tortuosity and very low maximum depth and mean angle.

There were several burrows produced by the two different millipede species that were found to be identical using the Bray Curtis analysis. Most of these occurrences $(78 \%)$ were between burrows of the same architecture (Appendix 8). Other identical burrows, however, were assigned to different architectures (Appendix 5). These mostly (71\%) resulted from comparisons of vertical and subvertical burrows; for example, a vertical burrow of Floridobolus penneri with a spiral in its midsection (FP7D) was identical to three subvertical burrows of Narceus americanus (NA21B, NA30D, NA30E) (Appendix 5). A few occurrences of identical burrows between the other architectures did occur, such as between a vertical burrow of $N$. americanus with a helical structure at the end (NA30F) and two J-shaped burrows of F. penneri $(\mathrm{FP} 2 \mathrm{H}$, FP2I) as well as between a helical burrow (NA33C) of $N$. americanus and a vertical (FP7B) and a subvertical burrow (FP3D) of F. penneri (Appendix 5).

Spirobolid and Spirostreptid Burrows. Burrows produced by two species of millipedes of the Order Spirostreptida, Orthoporus ornatus, and Archispirostreptus gigas, (Hembree, 2009) had a moderate level of similarity (0.7) to those of the species of the Order Spirobolida from this study (Figures 14, 18, 19; Appendices 9, 10, 11, 12, 15). Burrows of $O$. ornatus, however, were found to be more similar to those of the spirobolid millipedes than the burrows of $A$. gigas. All but one burrow of $O$. ornatus 


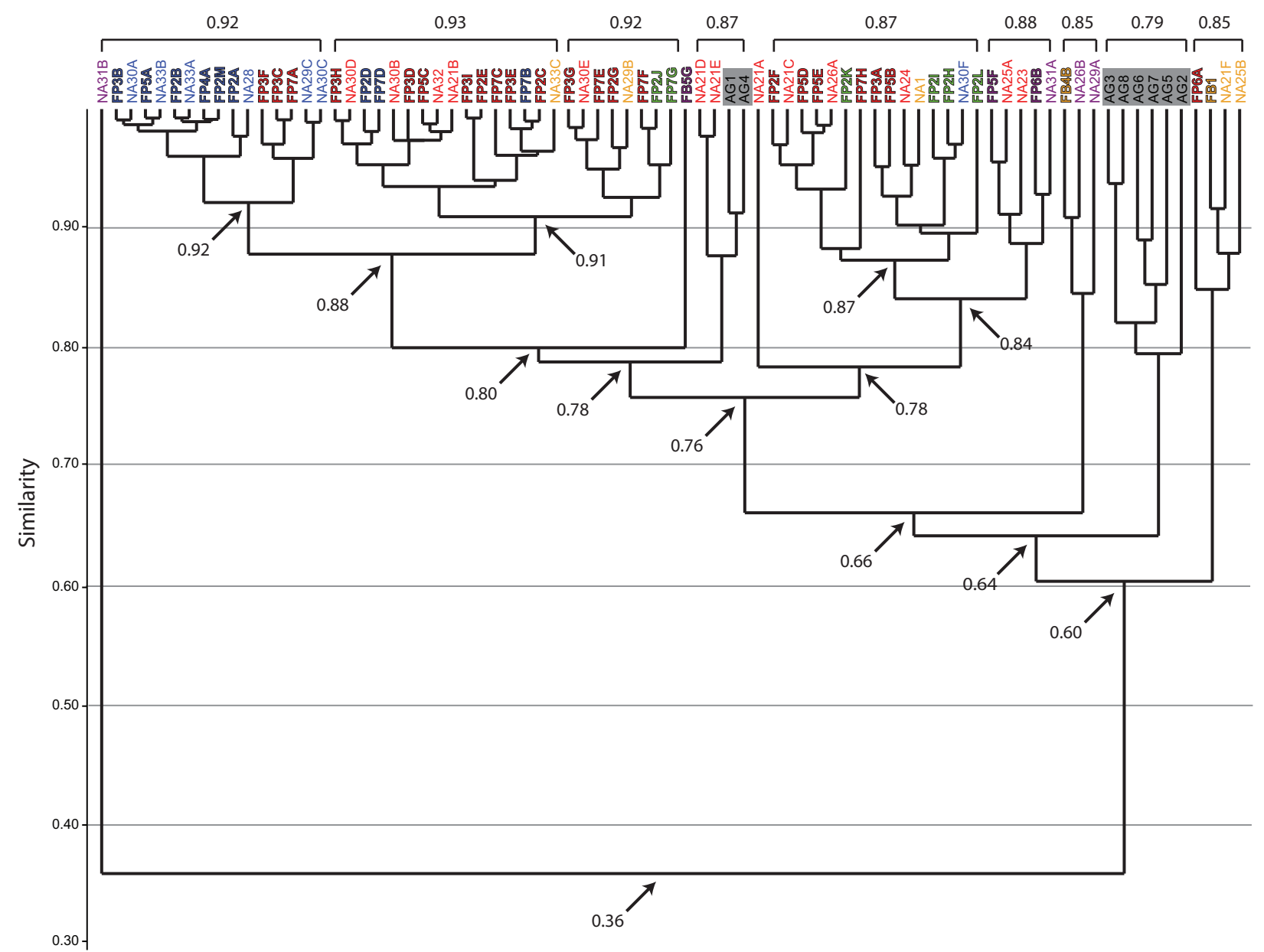

FIGURE 19. Bray Curtis cluster diagram comparing the 71 burrow casts produced by Narceus americanus (NA) and Floridobolus penneri (FP) with 8 burrow casts produced by Archispirostreptus gigas (AG; shaded gray) from Hembree (2009). Burrow cast ID numbers are color coded by architecture: red = subvertical burrow; blue = vertical burrow; orange = helical burrow; purple = O-shaped burrow; green = J-shaped burrow. Branch points near the top of the diagram indicate higher levels of similarity. Key branch points are marked with their similarity value (at arrows). Values $>0.80$ indicate high similarity, $0.79-0.60$ indicate moderate similarity, and values of 0.59 or less indicate dissimilarity. Groups of burrow casts found to be most similar are marked with brackets and their level of similarity.

(OO10) had a similarity of $>0.75$ when compared to the burrows of Narceus americanus and Floridobolus penneri (Figure 18). As a result, the burrows of $O$. ornatus grouped closely with the burrows of the other two millipedes, typically associated by primary architectures (Figure 18). Most $(75 \%)$ burrows of $A$. gigas, however, had a lower level of similarity (0.64) and occurred in a discrete cluster (Figure 19). When compared to each other, burrows of $O$. ornatus and $A$. gigas were mostly separated into two distinct groups at moderate levels of similarity (0.71) (Figure 20; Appendix 13)

Within each species, the burrows of both Orthoporus ornatus and Archispirostreptus gigas had a mean similarity value of 0.8 (Figure 14). When each species' suite of burrows were com- pared to each other, Narceus americanus $(n=29)$, Floridobolus penneri $(n=42)$, and O. ornatus $(n=13)$ burrows all had high similarity values $(0.8)$ (Figures 14, 18; Appendices 9, 10, 15). Burrows produced by $A$. gigas $(n=8)$, however, were found to be only moderately similar to $N$. americanus (0.6), F. penneri (0.6), and O. ornatus (0.7) (Figures 14, 19, 20; Appendices 11, 12, 13, 15). The four species of millipedes did produce many similar burrow architectures that could be compared. Vertical, subvertical, and J-shaped burrows of $N$. americanus, $F$. penneri, and $O$. ornatus had high similarity values (0.9-0.8) (Figures 14, 18; Appendices 9, 10). Helical burrows produced by $A$. gigas $(n=4)$, however, had only a moderate similarity (0.6) to those of $N$. americanus and F. penneri (Figures 14, 19; Appen- 


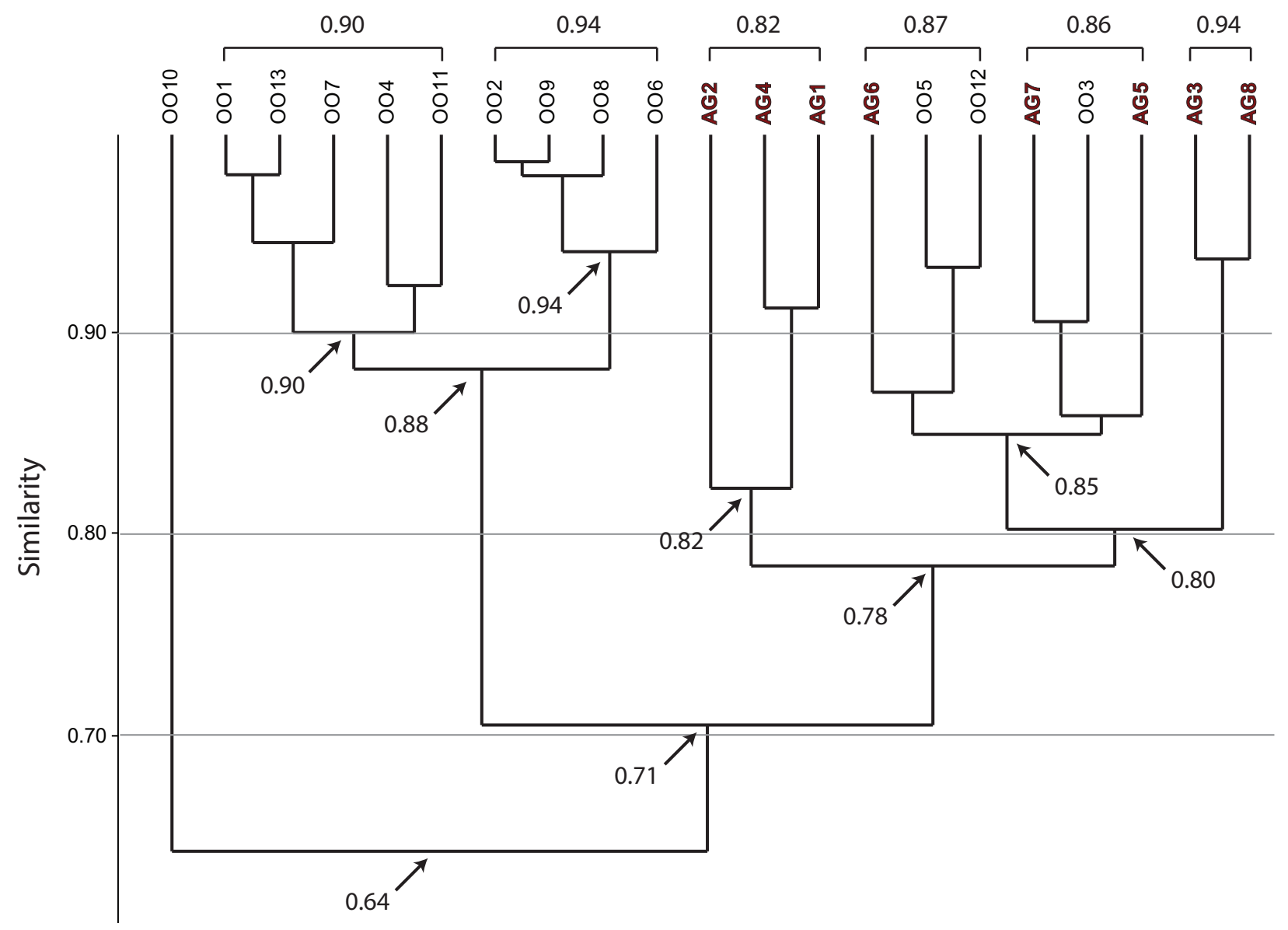

FIGURE 20. Bray Curtis cluster diagram comparing the 21 burrow casts produced by Orthoporus ornatus (OO) and Archispirostreptus gigas (AG). Branch points near the top of the diagram indicate higher levels of similarity. Key branch points are marked with their similarity value (at arrows). Values $>0.80$ indicate high similarity, $0.79-0.60$ indicate moderate similarity, and values of 0.59 or less indicate dissimilarity. Groups of burrow casts found to be most similar are marked with brackets and their level of similarity.

dices 11, 12). Orthoporus ornatus and $A$. gigas both produced $U$-shaped $(n=3)$ and sinuous $(n=3)$ burrows that were not produced by either $N$. americanus or F. penneri. The U-shaped burrows of both species, however, had a high mean similarity (0.8) to the J-shaped burrows of $F$. penneri (Figure 14, 18, 19; Appendices 10, 12). The sinuous burrow $(n=1)$ of $O$. ornatus also had a high similarity $(0.9)$ to the J-shaped burrows (Figure 14, 18; Appendix $10)$. The sinuous burrows of $A$. gigas $(n=2)$, however, had only moderate similarity $(0.7-0.6)$ to most other burrow architectures and were dissimilar to the O-shaped burrows (Figure 14, 19; Appendices 11, 12).

Four Orthoporus ornatus burrows were found to be identical to burrows of Floridobolus penneri (Appendix 10). Three vertical burrows of O. ornatus $(004,006,008)$ were identical to a vertical burrow with a helical mid-section (FP7D), a vertical (FP2D) and subvertical burrow (FP3G), and a verti- cal burrow (FP4A), respectively (Appendix 10). In addition, a J-shaped burrow of $O$. ornatus (OO11) was identical to two subvertical burrows (FP3I, FP2E) and one J-shaped burrow (FP2J) (Appendix 10).

\section{Environmental Conditions and Burrow Morphology}

Despite differences in mean maximum burrow depth measured from casts, percent clay content and moisture conditions had no significant effect on any of the eight quantitative measurements tested (complexity, tortuosity, mean circumference, total length, mean width, mean height, maximum depth and mean slope). The Spearman's rank correlation tests produced Rs values between -0.390.42 for percent clay composition and $-0.33-0.46$ for moisture content compared to the burrow measurements (Table 4; Appendices 14, 15). These 
TABLE 4. Rs values of the Spearman's rank correlation tests between sediment clay and moisture content and quantitative burrow properties. The number of burrow openings rarely varied between burrow casts and were, therefore, excluded from the analysis.

\begin{tabular}{lcccccccc} 
& \multicolumn{9}{c}{ Narceus americanus } \\
& Length & Depth & Width & Height & Circum. & Slope & Complexity & Tortuosity \\
\cline { 2 - 9 } Clay & -0.31 & -0.10 & 0.01 & 0.42 & -0.13 & -0.39 & -0.28 & -0.30 \\
Moisture & -0.22 & -0.33 & -0.13 & 0.41 & 0.04 & 0.46 & -0.10 & -0.11
\end{tabular}

\begin{tabular}{lcccccccc} 
& \multicolumn{9}{c}{ Floridobolus penneri } \\
& Length & Depth & Width & Height & Circum. & Slope & Complexity & Tortuosity \\
\cline { 2 - 9 } Clay & 0.15 & 0.19 & 0.24 & 0.36 & 0.22 & 0.03 & -0.17 & 0.14 \\
Moisture & 0.03 & -0.07 & 0.26 & -0.01 & 0.04 & -0.01 & 0.09 & 0.11
\end{tabular}

values indicated that the millipedes did not significantly change their behavior in a way that was reflected in their quantitative burrow morphology. There was, however, a difference in the diversity of burrow types (primary architectures and modifications) produced in the different sediments. Floridobolus penneri produced the highest diversity of burrow types in a $100 \mathrm{~S}$ sediment $(n=6)$ and in 25Sa/75S sediments $(n=5)$ under higher $(\geq 50 \%)$ moisture conditions. Narceus americanus produced the most types in 500/50S sediments $(n=4)$ under both low and high (30-40\%) moisture conditions.

\section{DISCUSSION}

Species and even individuals can produce many different burrow architectures and different species can produce the same architectures (Bromley, 1996). Burrow morphology is generally influenced by organism morphology, behavior, and their response to variations in environmental stimuli, including biotic and abiotic factors. Narceus americanus and Floridobolus penneri produced four and five distinct burrow architectures, respectively, under varying sediment compositions and moisture conditions. All but one of these architectures was produced by both species. Comparison of these burrows produced by millipedes of the Order Spirobolida with those produced by millipedes of the Order Spirostreptida indicated that some burrows were very similar while others were unique to one of the orders or species.

\section{Organism and Burrow Morphology}

Burrows produced by the millipede species in this study and those studied by Hembree (2009) were moderately to highly similar (0.6-0.9); how- ever, despite variation in other quantitative burrow properties, width-to-height ratio was found to be relatively constant $(1.00-1.14)$ among all of the burrow casts. The mean width and mean height of burrows produced by Archispirostreptus gigas, for example, were nearly three times those of burrows produced by the other three species. This relationship was also present in the body diameters of individuals used: Narceus americanus: $3-7 \mathrm{~mm}$, Floridobolus penneri: 6-9 mm, Orthoporus ornatus: 4-7 mm (Hembree, 2009), and A. gigas: 15$30 \mathrm{~mm}$ (Hembree, 2009). Despite these differences in body and burrow size, the mean width-toheight ratios of burrows produced were almost identical. The close relationship between millipede body shape and burrow morphology suggests that the width-to-height ratio of 1.00-1.14 may be useful to help support the interpretation of fossil burrows as being produced by millipedes with a juloid morphotype.

The mean widths of the tunnels and shafts, but not chambers, were $30-51 \%$ larger than the mean diameter of the millipedes (Narceus americanus: $51 \%$, Floridobolus penneri: $36 \%$, Orthoporus ornatus: $46 \%$, Archispirostreptus gigas: $30 \%$ ). Even though millipede burrow morphology is closely related to body morphology, this difference in diameter indicates that burrow size is not an exact match to the tracemakers' size. The variations in burrow width are likely the result of the burrowing techniques used by the individuals. Both compaction and excavation can be used to make burrows with larger dimensions than the tracemaker. This increase in burrow size relative to the tracemaker is well illustrated by the construction of chambers (Figures 5.1, 9.5, 11.3). Additionally, when burrowing, millipedes are not restricted to a single orientation. Millipedes are slightly taller than 
they are wide, so the shift from burrowing rightside-up to sideways causes shifts in the cross-sectional size of the burrow.

Wedge-shaped indentations and curving portions of some chambers represent indentations of the millipede's body (Figure 7.1-2) and are similar in appearance to the anal segment of the millipede. The occurrence of fecal pellets within these chambers suggests that they were occupied for extended periods of time (Figure 7.3-5). The millipedes were only observed to burrow head first, and the anal segment was never involved in the burrowing process. Therefore, the indentations are most likely body impressions rather than an expression of behavior. It is likely that the millipedes were buried under sediment as a result of gravitational collapse of the burrow; the millipedes then re-excavated the burrow and moved to the surface, leaving behind an indentation of the back portion of its body. Curved portions of burrow chambers likely represent the individual curling around itself (Figures 5.1, 9.5, 11.3) or points where the millipede turned around (Figures 9.4, 10.4), requiring the formation of a larger void space.

\section{Burrow Morphology and Behavior}

As the direct result of behavioral interaction with a medium (Bromley, 1996), variations in burrow morphology can reflect differences in organism behavior. Burrows and surficial traces produced by Narceus americanus and Floridobolus penneri represent dwelling, feeding, and locomotive behaviors. Over the course of these experiments, $N$. americanus and $F$. penneri continually attempted to move outside the bounds of the terraria while on the surface, resulting in the production of trails outlined by large grains. This continual locomotion behavior was possibly part of a search for a more preferential habitat, food, or mates. Extant millipedes, for example, are known to migrate in large numbers in response to detrimental changes in the environment (Kania and Tracz, 2005). Additional trails or furrows were produced when millipedes began to burrow but then moved laterally prior to being deep enough to produce a structurally stable burrow (Figure 6.1-2). This type of trail is evidence of the head-ramming compaction technique used by both species.

Shallow burrows that were never fully occupied were considered probing structures. These burrows were cone shaped, usually $<3 \mathrm{~cm}$ long, and represented abandoned attempts at burrow- ing. These structures were limited to the vertical and subvertical architectures.

The millipede species used in this study were cathemeral, but primarily nocturnal. In response to light stimuli, the millipedes commonly either began to immediately burrow or entered an open burrow. Burrows that were occupied for short periods $(<12$ hours) were usually produced as a means of avoiding light exposure, likely to avoid evaporative desiccation and predation. These temporary dwellings generally had a total length that was long enough to accommodate the individual's entire body (4.0$12.5 \mathrm{~cm}$ ), but had a relatively shallow maximum depth $(1.1-8.2 \mathrm{~cm})$. Vertical, subvertical, J-shaped, helical, and Type-2 O-shaped structures were produced for this purpose.

Structures that were occupied for longer periods of time ( $>2$ days) generally had a greater total length $(7.7-33.0+\mathrm{cm})$ and maximum depth (5.7$33.0 \mathrm{~cm}$ ) in both observed in situ and cast burrows than burrows occupied for shorter periods. In addition, burrows that were occupied for long periods commonly contained more fecal pellets and had chambers. These burrows likely served a dual purpose of dwelling and feeding. Fecal pellets were commonly composed of clay material as well as organics indicating the millipedes were consuming the soil, although this was not directly observed. Narceus americanus eats and dwells in rotting wood (Ausmus, 1977), therefore, wood splinters or the organic coconut fiber present in the soil mixture may have been an additional food source for these millipedes. The occurrence of fewer fecal pellets in burrows produced in the sandy sediments supports this conclusion. All architectures, except for Type-2 O-shaped structures, were produced for long-term occupation.

Burrows occupied for long periods were often used for molting or entering a state of torpor (i.e., aestivation) likely as a result of adverse surface conditions. During the experimental trials, for example, there was a point at which surface activity decreased in all millipedes. On average, experiments conducted between August 15th and February 1st took eight times longer past the expected duration than those conducted at other times of the year (Table 1). Laboratory conditions were kept relatively constant throughout the year and this decreased surface activity also occurred in holding terraria. It seems likely, therefore, that there were other seasonal cues to remain underground for long periods. Burrows used for molting or aestivation contained a chamber large enough for an entire individual; usually having a greater 
width than height. Millipedes were found as shallow as $4 \mathrm{~cm}$ and as deep as $33 \mathrm{~cm}$ after being inactive for more than two weeks, indicating that burrows produced for this behavior can be at any depth range.

Branches were never longer than a few $\mathrm{cm}$ in Narceus americanus or Floridobolus penneri burrows. Most branches (9 of 10 ) were at a $10-70^{\circ}$ angle from the horizontal off of the main shaft and occurred as a modification to a primary architecture. Since these branches did not continue any great distance and were only present in $11 \%$ of burrows, it is unlikely that they served any functional purpose. Their length and branching angle suggest that these were likely probing structures similar to abandoned burrows observed at the surface.

\section{Burrow Morphology and Sediment Properties}

An overall decrease in the mean maximum depth of burrows produced in sandy sediment was observed, however, neither sediment composition nor moisture content had a statistically significant impact on morphology (Table 4; Appendices 14, 15). Millipede burrow morphology is, therefore, interpreted to be controlled primarily by organism morphology and behavior. Architectural diversity of the burrows produced, however, did appear to be affected by sediment properties. In general, individuals of both species had more difficulty burrowing in sandy sediments, and collapse was more common as the surface of these sediments dried. Sediments containing organic matter and higher amounts of clay were able to hold moisture longer and, therefore, burrow stability was maintained for longer periods.

\section{Millipede Burrows: Function and Similarity}

The four millipede species discussed in this study are different in habitat, body size, and burrowing techniques. Despite these differences, burrows produced by Narceus americanus, Floridobolus penneri, Orthoporus ornatus, and Archispirostreptus gigas were not found to be dissimilar based on the 10 quantitative measurements of burrow morphology. It was determined that the burrow morphology of $N$. americanus and $F$. penneri was primarily controlled by organism morphology and behavior. Similar conclusions were drawn for O. ornatus and A. gigas by Hembree (2009). The similarities observed between burrows produced by these four species help to support this conclusion. The morphology of juloid millipedes restricts the burrowing techniques that they can use and the cross-sectional shape of the burrows they produce. With the differences in the sizes of the species being compared, it could be expected that the burrows of $A$. gigas would be less similar to those produced by the other species. The BrayCurtis similarity test results obtained did show that $A$. gigas burrows were the least similar to any of the other species. This difference, however, cannot solely be attributed to the difference in the size of the millipedes. An additional Bray-Curtis similarity test was conducted after the burrow measurements that were most closely related to size of the burrow cast (mean height, mean width, and mean circumference) were removed. The mean similarity of $A$. gigas burrows compared to the burrows of the other species improved, but still had the lowest similarity value compared to any other species (0.7). Hembree (2009) determined that the function of burrows produced by $A$. gigas was for feeding and locomotion while $O$. ornatus used their burrows primarily for refuge and dwelling. The function of burrowing behavior observed in $N$. americanus and $F$. penneri was determined to be related to dwelling, refuge from adverse conditions, feeding, and molting. Similarity values between burrows produced by different millipede species were the highest between $N$. americanus, F. penneri, and $O$. ornatus, even after the organism-size related measurements were removed. A conclusion, therefore, is that the similarity of the burrows produced by these millipedes is most strongly related to the function of the burrow.

\section{SIGNIFICANCE}

\section{Recognition of Juliform Millipede Burrows in the Fossil Record}

In order to properly interpret trace fossils, a thorough understanding of modern tracemakers and behaviors is required. The importance of understanding modern tracemakers is increased when the morphology of the species under investigation is suspected to have undergone few changes over geologic time. The ring-form body plan of the superorder Juliformia was present in the Early Devonian (Wilson, 2006b; Cong et al., 2009), but despite the longevity of a body plan that is well adapted to burrowing, few fossil burrows have been attributed to millipedes. This lack of trace fossil evidence is likely due to a lack of recognition rather than true absence. The proper description of extant millipede burrows using identifiable characteristics may allow millipede burrows to be accurately identified in the fossil record. 
In order to facilitate the identification of juliform millipede burrows in the fossil record, the definable characteristics of the burrows produced by two modern spirobolid millipedes, Narceus americanus and Floridobolus penneri, are summarized below using the suggested morphological ichnotaxobases as defined by Bertling et al. (2006).

Architecture. Spirobolid millipede burrows consist of vertical, subvertical, J-shaped, helical, and horizontally or vertically oriented O-shaped burrows with one or two surface openings. Branching is uncommon; branches are generally shorter than the primary burrow shaft and can occur within all burrow architectures. Chambers are most commonly present at the end of a burrow, but may be anywhere within the burrow. Chambers can be horizontally and vertically expanded and are 2-3 times the diameter of the primary burrow shaft or tunnel.

Overall Shape. Shafts and tunnels are nearly circular in cross-section with a mean width-to-height ratio of 1.1. Burrow heights and widths commonly increase toward burrow entrances and decrease near the end of the burrow. Chambers vary in size and shape, but commonly outline the individual. Burrows vary from straight to sinuous depending on the presence of branches, chambers, and helical additions. The most distinct architecture, Oshaped burrows, consist of a primary shaft that follows either an elliptical, circular, or teardropshaped path connecting back to itself. J-shaped burrows follow a similar path but stop just prior to connecting back to the primary shaft.

Orientation. Burrows are oriented from $20-90^{\circ}$ to the horizontal. Branches are typically oriented 12$60^{\circ}$ from the primary shaft or tunnel.

Internal Structure. Spirobolid millipede burrows are constructed using excavation and compaction and may have a compressed lining depending on the substrate. Burrows are passively filled, but may also contain fecal pellets.

Surficial Features. Floors can be irregular, but ceilings and walls are relatively smooth. Chambers and burrow end points may have wedge-shaped imprints from the anal segment of the millipede. Fecal pellets are commonly incorporated into the walls and floor of the burrow and can be in groups, straight lines, or randomly placed.

\section{Paleontologic and Paleoecologic Significance}

While millipede ecology, physiology, and behavior have been well studied and their burrowing behavior is widely known, little work has been done to understand their burrow morphology (Hembree, 2009). Many modern soil organisms are poorly understood, particularly in their trace-making abilities. These organisms tend to have a low preservation potential since they often reside in an environment where organic matter is quickly broken down, recycled, or fragmented through bioturbation (Retallack, 2001b; Hättenschwiler et al., 2005; Zhang et al., 2008; Shear and Edgecombe, 2010). Therefore, body fossils of these organisms may not accurately represent true biodiversity or abundance. In addition, terrestrial organisms are exposed to extreme variances in temperature, precipitation, evaporation, and habitats. While body fossils can be used to interpret environmental conditions based on a modern analog, organisms can change over time and become adapted to different conditions while not significantly or predictably changing their morphology. Conversely, behaviors are considered relatively constant through time. As direct representations of behaviors, modern traces are well suited to be used as proxies for past environmental conditions if similar trace fossils are found (Bromley, 1996). Neoichnological studies are vital to understanding historical abundance and how environmental conditions can affect burrow morphology.

Five different burrow architectures were produced by Narceus americanus and Floridobolus penneri with only one architecture specific to one species. Not only do the two species produce similar burrow architectures, but individuals were found to produce multiple burrows of different architectures. While individuals were surface active, burrowing was continuous. If this level of burrowing activity has not changed over millipede evolution, burrows produced by millipedes should be much more common in the fossil record than is currently known. Furthermore, if all burrows produced by millipedes in the fossil record were identified, millipede abundance may be over-estimated by burrow abundance. The sizes of the burrows produced in this study were closely related to the sizes of the tracemakers. This relationship, however, can only be used to estimate the maximum size of the individual. Millipedes used excavation and compressive techniques to construct shafts, tunnels, and chambers that were approximately $30-51 \%$ larger than the width of the individuals used in this study.

Millipede burrows have a moderate to high preservation potential. Millipede burrows produced in this study were all open to the surface, primarily produced via compacting and stabilizing the surrounding sediment, and were commonly able to penetrate the sediment to depths greater than 20 $\mathrm{cm}$. The open burrows could be passively filled by 
contrasting sediment carried by floods and wind. Open subsurface burrows not passively filled by surface sediment could be filled as a result of water infiltration with minerals precipitating in the void spaces or the accumulation of translocated clays. The millipede burrows, whether open or passively filled, were more permeable than the surrounding sediment. Burrows produced against the side of the terrarium served as conduits for water infiltration when the surface of the terrarium was sprayed. High permeability also increases the likelihood of burrow visibility as diagenetic redox processes highlight the burrow fill (Bromley, 1996).

Burrowing in millipedes may have been an early adaptation since the ring-form body plan and diplosegment condition were present in Devonian fossils (Wilson and Anderson, 2004, Wilson, 2006b; Cong et al., 2009). Widely accepted as one of the first macrofauna on land, it is possible that the accurate identification of millipede burrows would increase the temporal and geographic range of the Diplopoda.

\section{Paleopedologic and Paleoenvironmental Significance}

As air-breathing detritivores, millipedes require a terrestrial environment with decomposing vegetation. This necessitates that where burrows of millipedes are present, some form of vegetation and terrestrial conditions must be present. Fossil millipede burrows may then be used as a proxy for aerated continental deposits with surface vegetation.

Differences in the morphology of millipede burrows compared in this study have shown that millipede burrows constructed for different purposes may be discernible. If burrows constructed for the purpose of environmental isolation (i.e., aestivation) can be identified in the fossil record, it may be possible to differentiate seasonal climatic conditions from more stable ones. Narceus americanus is widely distributed and is primarily found in temperate forests in the eastern United States (O'Neill, 1968a, 1968b; Ausmus, 1977; Shelley et al., 2006). The soils of its habitat are subject to yearly freeze-thaw cycles with frozen soil extending to $11.7 \mathrm{~cm}$ in the absence of snowpack and 2.9 $\mathrm{cm}$ in the presence of snowpack in New York (Vermette and Christopher, 2008). Burrowing to depths greater than $12 \mathrm{~cm}$ may, therefore, be a behavioral adaptation to avoiding freezing soil conditions. The Florida scrublands are known for soils with low water retention and low soil moistures during dry seasons (Weekley et al., 2007). In this environ- ment Floridobolus penneri must burrow to depths of $>50 \mathrm{~cm}$ to encounter soil moisture levels of only $10 \%$ (Weekley et al., 2007). Maximum burrow depth may, therefore, be directly related to survivability during extreme climate variability. The periods of low activity observed in the laboratory correlate with seasonally cold temperatures and seasonally dry conditions in $N$. americanus and $F$. penneri habitats, respectively. With these strong adaptive requirements, it is not surprising that similarity tests would indicate that burrow function may play a stronger role in burrow morphology than specific environmental factors.

The requirement of many millipedes for calcium to maintain their cuticles may be linked to a preference toward calcium-rich environments (Kime and Golovatch, 2000). Millipedes are likely able to consume the calcium needed directly from calcium-rich soils and plants. This requirement restricts where millipedes may have made their first landward incursions. The earliest millipedes may have fed on bryophytes that grew on limestone, lichens that grew near the seashore, or fungi, all of which have been shown to have elevated calcium contents in modern studies (Cromack et al., 1977; Bates, 1982; Rhoades, 1999). Coprolites from the Early Devonian attributed to millipedes contained fragments of nematophytes, which may represent another nutritional source for early Diplopoda (Edwards et al., 2012).

Numerous studies have been conducted on the effect millipedes have on soil formation and nutrient cycling as detritivores (Ausmus, 1977; Crawford, 1992; Bonkowski et al., 1998; Cárcamo et al., 2000; Fujimaki et al., 2010), but little has been done to determine how millipede burrows affect soil formation. The burrowing techniques employed by the millipedes used in this study allowed for the construction of open channels deep into the soil subsurface. These burrows allow oxygen, water, and other organisms access to deeper soil horizons than they would normally be able to reach (Hole, 1981; Wilkinson et al., 2009). As a result, the physical and chemical processes that break down minerals within the soil are accelerated (Schaetzl and Anderson, 2005). In addition, these millipedes were found to consume plant material above and below the surface and deposit their fecal pellets both on the surface and in their burrows. This physical and chemical breakdown of plant material allows these nutrients to become available to soil microbes and bacteria (Schaetzl and Anderson, 2005). The overall effect would be an increase in the organic content of the soil. 
Millipede fecal pellets are known to be an important food source for some earthworms (Bonkowski et al., 1998). Farfan (2010) even suggested that through the act of self-coprophagy, millipedes may be able to survive instances of human mediated accidental transportation with little sediment present. Some millipedes also store eggs within fecal pellets in their burrows, but no chambers constructed in this study were observed to have been used for this purpose. Accumulations of fecal pellets within the millipede burrows may, therefore, be used as a nutrient source to help sustain the individual or its young when remaining underground for long periods. The growth of fungi and bacteria on the fecal pellets may even be considered a "farming" technique if further studies support intentional cultivation of these organisms from fecal pellets. Large accumulations of fecal pellets suggested as possibly being produced by millipedes have been reported from within fossil fern stems of the Upper Pennsylvanian in Ohio (Rothwell and Scott, 1983).

Millipedes are widely distributed, therefore, their ichnofossils would likely be found in all soil types except for Gelisols. Their preference for calcic environments may mean that calcium-rich soils would be the most likely habitat. Waterlogged and ever-wet soils, however, are unlikely to contain millipede burrows. Being detritivores, millipedes require decomposing plant material for consumption meaning that, along with millipede burrows, rhizoliths should be common. The long temporal range of millipedes places them in time periods with all other terrestrial macrofauna. The co-occurrence of millipede burrows with many other soildwelling annelid, insect, arachnid, reptile, amphibian, and mammal burrows is very likely.

\section{CONCLUSIONS}

Understanding ancient ecosystems begins with the study of the modern. Some aspects of modern ecosystems have been well studied, but soil animals and their traces have been widely overlooked. Neoichnological studies are required to address the key aspects of continental trace fossils such as the identity of their tracemakers and their associated behaviors. Despite their high diversity, abundance, vital role in modern ecosystems, and wide geographic and temporal range, very few studies have been conducted on the trace-making abilities of millipedes.

Narceus americanus produced four different burrow architectures. These architectures included vertical, subvertical, helical, and O-shaped bur- rows. Floridobolus penneri produced all of the architectures of $N$. americanus in addition to $\mathrm{J}$ shaped burrows. Burrow shafts and tunnels produced by the millipedes in this study were usually 2-3 $\mathrm{mm}$ larger than the individual indicating that burrow size is an overestimation of body size. Chambers were sometimes three times larger than the individual and varied in shape and size.

When burrows produced by the species used in this study were compared to burrows produced by Orthoporus ornatus and Archispirostreptus gigas in a similar study (Hembree, 2009) using a nonparametric statistical analysis, the burrows were found to have some highly similar quantitative elements. A mean width-to-height ratio of 1.0-1.14 was found to be consistent among burrows produced by all four species of millipedes. Burrows produced by Narceus americanus, Floridobolus penneri, and O. ornatus were found to have similar mean widths, mean heights, and mean slopes. Burrows produced by the largest of the four millipedes, $A$. gigas, were found to be the least similar even when properties most related to the size of the tracemaker were removed from the analysis. This difference was ultimately attributed to differences in the functions of the burrows. Narceus americanus, $F$. penneri, and $O$. ornatus constructed burrows for dwelling and environmental isolation, whereas $A$. gigas produced burrows for feeding and locomotion. The quantitative morphologic characteristics used in this study were, therefore, able to differentiate millipede burrows produced by different orders and for different functions.

It is commonly expected that burrow morphology is controlled by environmental conditions such as sediment composition and moisture content. This study found that these environmental conditions had no significant effect on burrow morphology. Instead entrenched evolutionary behaviors may be the strongest control on millipede burrow morphology. This study, therefore, illustrates the importance of tracemaker body morphology and behavior in the morphology of burrows. Burrowing technique was also shown to have little effect on the similarity of burrows produced by millipedes with very similar body plans. While Narceus americanus and Floridobolus penneri both used a combination of compaction and small amounts of excavation, their burrows were found to be highly similar and even identical to burrows produced by Orthoporus ornatus that uses only an excavation technique. 
No terrestrial macrodetritivore has a longer history than millipedes. A large portion of their record, however, has been largely ignored. Thorough neoichnological studies on the traces produced by millipedes will aid in uncovering the true diversity and abundance of millipedes in the fossil record as well as allow for the production of better paleoecologic, paleoclimatic, and paleoenvironmental reconstructions from the continental rock record.

\section{ACKNOWLEDGEMENTS}

We thank the National Science Foundation (EAR-0844256) and the American Chemical Society Petroleum Research Fund (49387-UNI8) for their generous support provided to Dr. Hembree to conduct this research. Finally, we thank A. Durkee for her assistance in the Continental Ichnology Research Laboratory at Ohio University.

\section{REFERENCES}

Almond, J.E. 1985. The Silurian-Devonian fossil record of the Myriapoda. Philosophical Transactions of the Royal Society of London. Series B, Biological Sciences, 309:227-237.

Ausmus, B.S. 1977. Regulation of wood decomposition rates by arthropod and annelid populations. Ecological Bulletins, Soil Organisms as Components of Ecosystems, 25:180-192.

Bailey, P.T. and Kovaliski, J. 1993. Summer quiescent behavior of the millipede Ommatoiulus moreleti (Julida: Julidae). Journal of Zoology, 231:523-532.

Banerjee, B. 1967. Diurnal and seasonal variations in the activity of the millipedes Cylindroiulus punctatus (Leach), Tachypodoiulus niger (Leach) and Polydesmus angustus Latzel. Oikos, 18:141-144.

Bates, J.W. 1982. The role of exchangeable calcium in saxicolous calcicole and calcifuge mosses. New Phytologist, 90:239-252.

Bertling, M., Braddy, S.J., Bromley, R.G., Demathieu, G.R., Genise, J., Mikuláš, R., Nielsen, J.K., Nielsen, K.S.S., Rindsberg, A.K., Schlirf, M., and Uchman, A. 2006. Names for trace fossils: a uniform approach. Lethaia, 39:265-286.

Bonkowski, N., Scheu, S., and Schaefer, M. 1998. Interactions of earthworms (Octolasion lacteum), millipedes (Glomeris marginata) and plants (Hordelymus europaeus) in a beechwood on a basalt hill: implications for litter decomposition and soil formation. Applied Soil Ecology, 9:161-166.

Borrell, B.J. 2004. Mechanical properties of calcified exoskeleton from the neotropical millipede, Nyssodesmus python. Journal of Insect Physiology, 50:1121-1126.
Bromley, R.G. 1996. Trace Fossils; Biology, Taphonomy and Applications, 2nd edition. Chapman and Hall, London.

Cárcamo, H.A., Abe, T.A., Prescott, C.E., Holl, F.B., and Chanway, C.P. 2000. Influence of millipedes on litter decomposition, $\mathrm{N}$ mineralization, and microbial communities in a coastal forest in British Columbia, Canada. Canadian Journal of Forest Research, 30:817826.

Carrel, J.E. and Britt, E.J. 2009. The whip scorpion, Mastigoproctus giganteus (Uropygi: Thelyphonidae), preys on the chemically defended Florida scrub millipede, Floridobolus penneri (Spirobolida: Floridobolidae). Florida Entomologist, 92:500-502.

Cong, P., Xia, X., and Yang, Q. 2009. Monophyly of the ring-forming group in Diplopoda (Myriapoda, Arthropoda) based on SSU and LSU ribosomal RNA sequences. Progress in Natural Science, 19:12971303.

Crawford, C.S. 1992. Millipedes as model detritivores, p. 277-288. In Meyer, E., Thaler, K., and Schedl, W. (eds.), Advances in Myriapodology: Proceedings of the Eighth International Congress of Myriapodology. Universitätsverlag Wagner.

Cromack, K., Sollins P., Todd R.L., Crossley, Jr. C.A., Fender, W.M., Fogel, R., and Todd, A.W. 1977. Soil microorganism-arthropod interactions: fungi as major calcium and sodium sources, p. 78-84. In Mattson, W.J. (ed.), Role of Arthropods in Forest Ecosystems. Springer-Verlag, New York.

Crozier, W.J. 1924. On the critical thermal increment for the locomotion of a diplopod. The Journal of General Physiology, 7:123-136.

Dangerfield, J.M. and Chipfunde, L. 1995. Stress tolerance and burrowing behavior in the southern African millipede Alloporus uncinatus. Journal of Zoology, 236:17-27.

Dashtgard, S.E. 2011. Linking invertebrate burrow distributions (neoichnology) to physiochemical stresses on a sandy tidal flat: implications for the rock record. Sedimentology, 58:1303-1325.

Davis, R.C. 1978. Ecological Studies of Isopoda and Diplopoda in Dune Grassland. Unpublished PhD Dissertation, University of Leeds, Leeds, UK.

Davis, R.B., Minter, N.J., and Braddy, S.J. 2007. The neoichnology of terrestrial arthropods. Palaeogeography, Palaeoclimatology, Palaeoecology, 255:284307.

Edwards, D., Selden, P.A., and Axe, L. 2012. Selective feeding in an Early Devonian terrestrial ecosystem. Palaios, 27:509-522.

Eisner, T., Eisner, M., Attygalle, A.B., Deyrup, M., and Meinwald, J. 1998. Rendering the inedible edible: circumvention of a millipede's chemical defense by a predaceous beetle larva (Phengodidae). Proceedings of the National Academy of Sciences USA, 95:1108-1113. 
Enghoff, H., Dohle, W., and Blower, J.G. 1993. Anamorphosis in millipedes (Diplopoda) - the present state of knowledge with some developmental and phylogenetic considerations. Zoological Journal of the Linnean Society, 109:103-234.

Farfan, M. 2008. Ecological Effects of the Installation of an Underground Oil Pipeline on Millipede Communities in Hocking County, Ohio. Unpublished BS Thesis, The Ohio State University, Columbus, Ohio, USA.

Farfan, M. 2010. Some aspects of the ecology of millipedes (Diplopoda). Unpublished MS Thesis, The Ohio State University, Columbus, Ohio, USA.

Fujimaki, R., Sato, Y., Okai, N., and Kaneko, N. 2010. The train millipede (Parafontaria laminate) mediates soil aggregation and $\mathrm{N}$ dynamics in a Japanese larch forest. Geoderma, 159:216-220.

Galanes, I.T. and Thomlinson, J.R. 2011. Soil millipede diversity in tropical forest patches and its relation to landscape structure in northeastern Puerto Rico. Biodiversity and Conservation, 20:2967-2980.

Genise, J.F., Melchor, R.N., Archangelsky, M., Bala, L.O., Straneck, R., and Valais, S. 2009. Application of neoichnological studies to behavioural and taphonomic interpretation of fossil bird-like tracks from lacustrine settings: the Late Triassic-Early Jurassic? Santo Domingo Formation, Argentina. Palaeogeography, Palaeoclimatology, Palaeoecology, 272:143161.

Getty, P.R., Sproule, R., Wagner, D., and Bush, A.M. 2013. Variation in wingless insect trace fossils: insights from neoichnology and the Pennsylvanian of Massachusetts. Palaios, 28:243-258.

Golovatch, S.I. and Kime, R.D. 2009. Millipede (Diplopoda) distributions: a review. Soil Organisms, 81:565597.

Greyling, M.D., Van Aarde, R.J., and Ferreira, S.M. 2001. Seasonal changes in habitat preferences of two closely related millipede species. African Journal of Ecology, 39:51-58.

Hamer, J.M.M. and Sheldon, N.D. 2010. Neoichnology at lake margins: implications for paleo-lake systems. Sedimentary Geology, 228:319-327.

Hammer, Ø. and Harper, D. 2006. Paleontological Data Analysis. Blackwell Publishing, Massachusetts.

Hammer, Ø., Harper, D.A.T., and Ryan, P.D. 2001. Paleontological Statistics software package for education and data analysis. Palaeontologia Electronica, 4:1-9.

Hasiotis, S.T. 2007. Continental ichnology: fundamental processes and controls on trace fossil distribution, $p$. 268-284. In Miller, W. (ed.), Trace Fossils: Concepts, Problems, Prospects. Elsevier, Amsterdam.

Hättenschwiler, S., Tiunov, A.V., and Scheu, S. 2005. Biodiversity and litter decomposition in terrestrial ecosystems. Annual Review of Ecology, Evolution, and Systematics, 36:191-218.
Hembree, D.I. 2009. Neoichnology of burrowing millipedes: linking modern burrow morphology, organism behavior, and sediment properties to interpret continental ichnofossils. Palaios, 24:425-439.

Hembree, D.I. and Hasiotis, S.T. 2006. The identification and interpretation of reptile ichnofossils in paleosols through modern studies. Journal of Sedimentary Research, 76:575-588.

Hembree, D.I., Johnson, L.M., and Tenwalde, R.W. 2012. Neoichnology of the desert scorpion Hadrurus arizonensis: burrows to biogenic cross lamination. Palaeontologia Electronica 15.1.10A: 34 pp, 14.3MB; http://palaeo-electronica.org/content/2012-issue-1articles/192-neoichnology-of-scorpions.

Hoffman, R.L. 1963. New genera and species of Upper Paleozoic Diplopoda. Journal of Paleontology, $37: 167-174$

Hole, F.D. 1981. Effects of animals on soil. Geoderma, 25:75-112.

Jeram, A.J., Selden, P.A., and Edwards, D. 1990. Land animals in the Silurian: arachnids and myriapods from Shropshire, England. American Association for the Advancement of Science, 250:658-661.

Johnson, E.W., Briggs, D.E.G., Suthren, R.J., Wright, J.L., and Tunnicliff, W.P. 1994. Non-marine arthropod traces from the subaerial Ordovician Borrowdale Volcanic Group, English Lake District. Geological Magazine, 131:395-406.

Kalisz, P.J. and Powell, J.E. 2003. Effect of calcareous road dust on land snails (Gastropoda: Pulmonata) and millipedes (Diplopoda) in acid forest soils of the Daniel Boone National Forest of Kentucky, USA. Forest Ecology and Management, 186:177-183.

Kania, G. and Tracz, H. 2005. Mass occurrence and migration of Ommatoiulus sabulosus (Linnaeus, 1758) (Diplopoda, Julida: Julidae) in Poland. Peckiana, 4:57-66.

Kime, R.D. and Golovatch, S.I. 2000. Trends in the ecological strategies and evolution of millipedes (Diplopoda). Biological Journal of the Linnean Society, 69:333-349.

Manton, S.M. 1953. The evolution of arthropodan locomotory mechanisms, part 4: the structure, habits and evolution of the Diplopoda. Journal of the Linnean Society, Zoology, 42:299-368.

Manton, S.M. 1958. Habits of life and evolution of body design in Arthropoda. Journal of the Linnean Society, Zoology, 44:58-72.

Manton, S.M. 1961. The evolution of arthropodan locomotory mechanisms, part 7: functional requirements and body design in Colobognatha (Diplopoda), together with a comparative account of diplopod burrowing techniques, trunk musculature and segmentation. Journal of the Linnean Society, Zoology, 44:383462. 
Meadows, P.S. 1991. The environmental impact of burrows and burrowing animals - conclusions and a model, p. 327-338. In Meadows, P.S. and Meadows A. (eds.), The Environmental Impact of Burrowing Animals and Animal Burrows. Clarendon Press, Oxford.

Moeed, A. and Meads, M.J. 1985. Seasonality of pitfall trapped invertebrates in three types of native forest, Orongorongo Valley, New Zealand. New Zealand Journal of Zoology, 12:17-53.

O'Neill, R.V. 1967. Behavior of Narceus americanus (Diplopoda) on slopes and its ecological significance. American Midland Naturalist, 77:535-539.

O'Neill, R.V. 1968a. Adaptive responses to desiccation in the millipede, Narceus americanus (Beauvois). American Midland Naturalist, 81:578-583.

O'Neill, R.V. 1968b. Population energies of the millipede, Narceus americanus (Beauvois). Ecology, 49:803809.

O'Neill, R.V. 1969. Comparative desiccation tolerance in seven species of millipedes. American Midland Naturalist, 82:182-187.

Pisani, D., Poling, L.L., Lyons-Weiler, M., and Hedges S.B. 2004. The colonization of land by animals: molecular phylogeny and divergence times among arthropods. BMC Biology, 2:1; http://www.biomedcentral.com/1741-7007/2/1.

Retallack, G.J. 2001a. Scoyenia burrows from Ordovician paleosols of the Juniata Formation in Pennsylvania. Palaeontology, 44:209-235.

Retallack, G.J. 2001b. Soils of the Past. Blackwell Science, Oxford.

Retallack, G.J. and Feakes, C.R. 1987. Trace fossil evidence for Late Ordovician animals on land. Science, 235:61-63.

Rhoades, F.M. 1999. A review of lichen and bryophyte elemental content literature with reference to Pacific northwest species. United States Department of Agriculture, Forest Service.

Rolfe, W.D.I. 1985. Early terrestrial arthropods: a fragmentary record. Philisophical Transactions of the Royal Society of London. Series B, Biological Sciences, 309:207-218.

Rothwell, G.W. and Scott, A.C. 1983. Coprolites within marattiaceous fern stems (Psaronius magnificus) from the Upper Pennsylvanian of the Appalachian Basin, U.S.A. Palaeogeography, Palaeoclimatology, Palaeoecology, 41:227-232.

Sattman, D.A. 2006. The Biology of the Florida Scrub Endemic Millipede: Floridobolus penneri. Unpublished MS Thesis, University of Missouri-Columbia, Columbia, Mississippi, USA.

Schaefer, I., Norton, R.A., Scheu, S., and Maraun, M. 2010. Arthropod colonization of land: linking molecules and fossils in oribatid mites (Acari, Oribatida). Molecular Phylogenetics and Evolution, 57:113-121.

Schaetzl, R.J. and Anderson, S. 2005. Soils: Genesis and Geomorphology. Cambridge University Press, New York.
Shear, W.A. and Edgecombe, G.D. 2010. The geological record and phylogeny of the Myriapoda. Arthropod Structure and Development, 39:174-190.

Shelley, R.M., McAllister, C.T., and Medrano, M.F. 2006. Distribution of the millipede genus Narceus Rafinesque, 1820 (Spirobolida: Spirobolidae): occurrences in New England and west of the Mississippi River; A summary of peripheral localities and first records from Connecticut, Delaware, Maine, and Minnesota. Western North American Naturalist, 66:374-389.

Sierwald, P. and Bond, J.E. 2007. Current status of the myriapod class Diplopoda (millipedes): taxonomic diversity and phylogeny. Annual Review of Entomology, 52:401-420.

Smilek, K.R. and Hembree, D.I. 2012. Neoichnology of Thyonella gemmata: a case study for understanding holothurian ichnofossils. The Open Paleontology Journal, 4:1-10.

Stašiov, S. 2009. Millipede (Diplopoda) communities in mixed oak-hornbeam forest stands - effect of selected site factors. Polish Journal of Ecology, 57:785-792.

Stašiov, S., Stašiová, A., Svitok, M., Michalková, E., Slobodník, B., and Lukáčik, I. 2012. Millipede (Diplopoda) communities in an arboretum: influence of tree species and soil properties. Biologia, 67:945-952.

Thorez, A., Compere, P., and Goffinet, G. 1992. Ultrastructure and mineral composition of the tergite cuticle of the iulid millipede Ophyiulus pilosus (Myriapoda, Diplopoda), p. 63-70. In Meyer, E., Thaler, K., and Schedl, W. (eds.), Advances in Myriapodology: Proceedings of the Eighth International Congress of Myriapodology. Universitätsverlag Wagner.

Toye, S.A. 1966. The reactions of three species of Nigerian millipedes (Spirostreptus assiniensis, Oxydesmus sp., and Habrodesmus falx) to light, humidity and temperature. Entomologia Experimentalis et Applicata, 9:468-483.

Tuf, I.H., Tufová, J., Jeřábková, E., and Dedek, P. 2006. Diurnal epigeic activity of myriapods (Chilipoda, Diplopoda). Norwegian Journal of Entomology, 53:335344.

Vermette, S. and Christopher, S. 2008. Using the rate of accumulated freezing and thawing degree days as a surrogate for determining freezing depth in a temperate forest soil. Middle States Geographer, 41:68-73.

Villani, M.G., Allee, L.L., Díaz, A., and Robbins, P.S. 1999. Adaptive strategies of edaphic arthropods. Annual Review of Entomology, 44:233-256.

Walker, M.J., Stockman, A.K., Marek, P.E., and Bond, J.E. 2009. Pleistocene glacial refugia across the Appalachian Mountains and coastal plain in the millipede genus Narceus: evidence from population genetic, phylogeographic and paleoclimatic data. BMC Evolutionary Biology, 9:25; http://www.biomedcentral.com/1471-2148/9/25. 
Ward, P., Labandeira, C., Laurin, M., and Berner, R.A. 2006. Confirmation of Romer's Gap as a low oxygen interval constraining the timing of initial arthropod and vertebrate terrestrialization. Proceedings of the National Academy of Science, 103:16818-16822.

Weekley, C.W., Gagnon, D., Menges, E.S., OuintanaAscencio, P.F., and Saha, S. 2007. Variation in soil moisture in relation to rainfall, vegetation, gaps, and time-since-fire in Florida scrub. Ecoscience, 14:377386.

Wilkinson, M.T., Richards, P.J., and Humphreys, G.S. 2009. Breaking ground: pedological, geological, and ecological implications of soil bioturbation. Earth-Science Reviews, 97:257-272.

Wilson, H.M. 2005a. A new genus of archipolypodan millipede from the Coseley Lagerstätte, Upper Carboniferous, UK. Palaeontology, 48:1097-1100.
Wilson, H.M. 2005b. Zosterogrammida, a new order of millipedes from the Middle Silurian of Scotland and the Upper Carboniferous of Euramerica. Palaeontology, 48:1101-1110.

Wilson, H.M. 2006a. Aggregation behavior in juvenile millipedes from the Upper Carboniferous of Mazon Creek, Illinois. Palaeontology, 49:733-740.

Wilson, H.M., 2006b. Juliform millipedes from the Lower Devonian of Euramerica: Implications for the timing of millipede cladogenesis in the Paleozoic. Journal of Paleontology, 80:638-649.

Wilson, H.M. and Anderson, L.I. 2004. Morphology and taxonomy of Paleozoic millipedes (Diplopoda: Chilognatha: Archipolypoda) from Scotland. Journal of Paleontology, 78:169-184.

Zhang, D., Hui, D., Luo, Y., and Zhou, G. 2008. Rates of litter decomposition in terrestrial ecosystems: global patterns and controlling factors. Journal of Plant Ecology, 1:85-93. 


\section{APPENDIX 1.}

Video of Narceus americanus using a compaction burrowing technique in 500/50S sediment. For live videos, see palaeo-electronica.org/content/2014/709-neoichnology-of-spirobolids

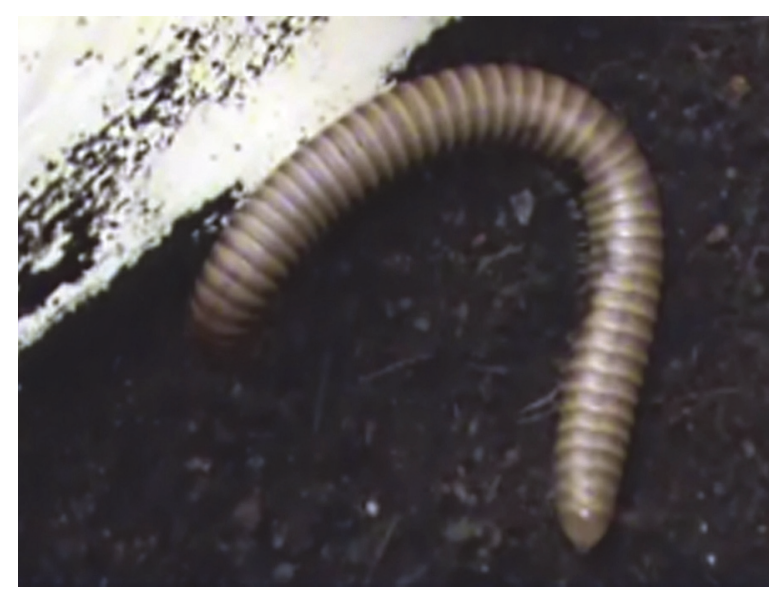

APPENDIX 2.

Video of Narceus americanus using an excavation burrowing technique in 50Sa/50S sediment. For live videos, see palaeo-electronica.org/content/2014/709-neoichnology-of-spirobolids

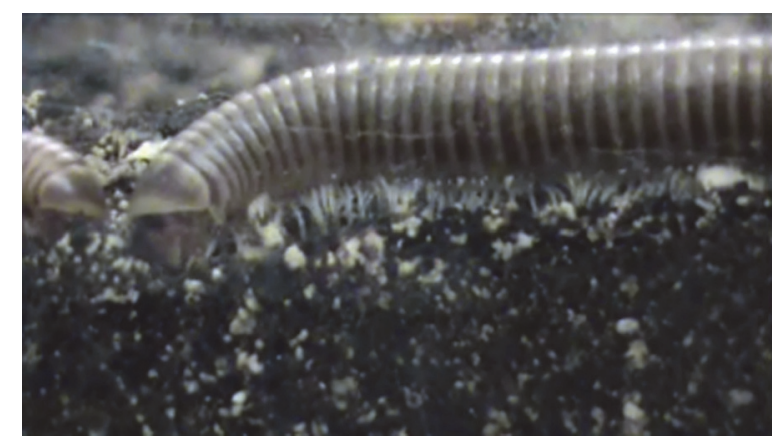

\section{APPENDIX 3.}

Video of Floridobolus penneri using a compaction burrowing technique in $50 \mathrm{Sa} / 50 \mathrm{~S}$ sediment. For live videos, see palaeo-electronica.org/content/2014/709-neoichnology-of-spirobolids

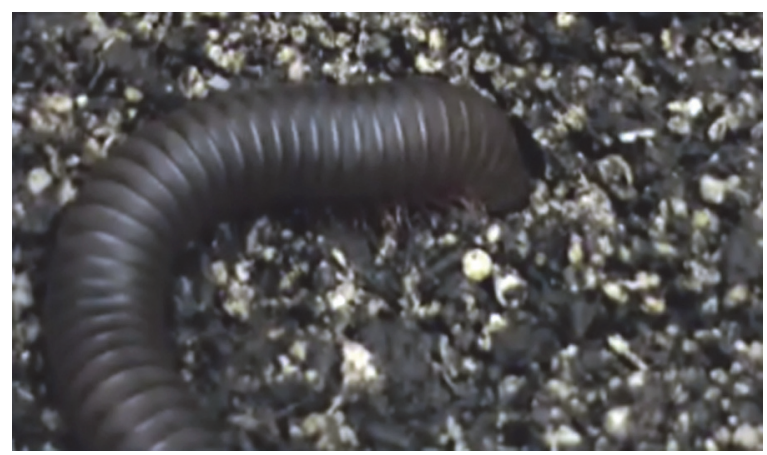




\section{APPENDIX 4.}

Video of Floridobolus penneri in the subsurface using an excavation burrowing technique to widen a burrow in 1000 sediment.

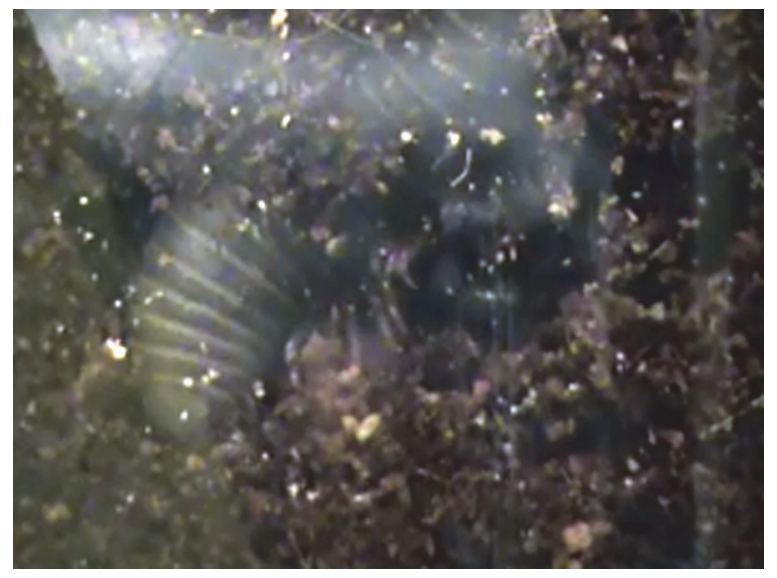




\section{APPENDIXES 5-13.}

See palaeo-electronica.org/content/2014/709-neoichnology-of-spirobolids for these appendixes in PDF format.

APPENDIX 5. Bray Curtis similarity matrix comparing all burrow casts produced in this study. From top-down and leftright; 29 Narceus americanus burrows (NA), 42 Floridobolus penneri burrows (FP), 13 Orthoporus ornatus burrows (OO prefix), and 8 Archispirostreptus gigas burrows (AG). Color bars along the sides and top indicate primary burrow architectures: Black $=$ subvertical burrow, gray $=$ vertical burrow, tan $=\mathrm{J}$-shaped burrow, brown $=$ helical burrow, magenta $=$ O-shaped burrow, dark green = sinuous burrow, yellow = U-shaped burrow. Colors inside the matrix indicate the level of similarity: green cells indicate identical burrows; blue cells indicate highly similar burrows; orange cells indicate moderately similar burrows; red cells indicate dissimilar burrows.

APPENDIX 6. Bray Curtis similarity matrices comparing the burrow architectures of Narceus americanus. 1) Comparison matrix of subvertical burrows. 2) Comparison matrix of vertical burrows. 3) Comparison matrix of helical burrows. 4) Comparison matrix of Type 1 and Type 2 O-shaped burrows. Colors inside the matrix indicate the level of similarity: green cells indicate identical burrows; blue cells indicate highly similar burrows; orange cells indicate moderately similar burrows; red cells indicate dissimilar burrows.

APPENDIX 7. Bray Curtis similarity matrices comparing the burrow architectures of Floridobolus penneri. 1) Comparison matrix of subvertical burrows. 2) Comparison matrix of vertical burrows. 3) Comparison matrix of J-shaped burrows. 4) Comparison matrix of Type 1 and Type 2 O-shaped burrows. 5) Comparison matrix of helical burrows. Colors inside the matrix indicate the level of similarity: green cells indicate identical burrows; blue cells indicate highly similar burrows; orange cells indicate moderately similar burrows; red cells indicate dissimilar burrows.

APPENDIX 8. Bray Curtis similarity matrices comparing the similar burrow architectures produced by Narceus americanus (NA) and Floridobolus penneri (FP). 1) Comparison matrix of subvertical burrows. 2) Comparison matrix of vertical burrows. 3) Comparison matrix of helical burrows. 4) Comparison matrix of O-shaped burrows. Colors inside the matrix indicate the level of similarity: green cells indicate identical burrows; blue cells indicate highly similar burrows; orange cells indicate moderately similar burrows; red cells indicate dissimilar burrows.

APPENDIX 9. Bray Curtis similarity matrix comparing burrows produced by Narceus americanus (NA) to burrows produced by Orthoporus ornatus (OO). Cells outlined in thick black lines in the matrix indicate comparison of burrows with the same architecture. 001, 007, and 0010 are subvertical burrows. 002, 004, 006, 008, and 009 are vertical burrows. $\mathrm{OO} 5$ is a sinuous burrow. $\mathrm{OO} 3$ is a U-shaped burrow. 0011-13 are J-shaped burrows. Colors inside the matrix indicate the level of similarity: blue cells indicate highly similar burrows; orange cells indicate moderately similar burrows; red cells indicate dissimilar burrows.

APPENDIX 10. Bray Curtis similarity matrix comparing burrows produced by Floridobolus penneri (FP) to burrows produced by Orthoporus ornatus (OO). Cells outlined in thick black lines in the matrix indicate comparison of burrows with the same architecture. 001, 007, and 0010 are subvertical burrows. 002, 004, 006, 008, and 009 are vertical burrows. $\mathrm{OO} 5$ is a sinuous burrow. $\mathrm{OO} 3$ is a U-shaped burrow. 0011-13 are J-shaped burrows. Colors inside the matrix indicate the level of similarity: green cells indicate identical burrows; blue cells indicate highly similar burrows; orange cells indicate moderately similar burrows; red cells indicate dissimilar burrows.

APPENDIX 11. Bray Curtis similarity matrix comparing burrows produced by Narceus americanus (NA) to burrows produced by Archispirostreptus gigas (AG). Cells outlined in thick black lines in the matrix indicate comparison of burrows of the same architecture. AG2 and AG8 are sinuous burrows. AG1, AG3, AG4, and AG6 are helical burrows. AG7 and AG5 are U-shaped burrows. Colors inside the matrix indicate the level of similarity: blue cells indicate highly similar burrows; orange cells indicate moderately similar burrows; red cells indicate dissimilar burrows.

APPENDIX 12. Bray Curtis similarity matrix comparing burrows produced by Floridobolus penneri (FP) to burrows produced by Archispirostreptus gigas (AG). Cells outlined in thick black lines in the matrix indicate comparison of burrows of the same architecture. AG2 and AG8 are sinuous burrows. AG1, AG3, AG4, and AG6 are helical burrows. AG7 and AG5 are U-shaped burrows. Colors inside the matrix indicate the level of similarity: blue cells indicate highly similar burrows; orange cells indicate moderately similar burrows; red cells indicate dissimilar burrows.

APPENDIX 13. Bray Curtis similarity matrix comparing burrows produced by Orthoporus ornatus (OO) to burrows produced by Archispirostreptus gigas (AG). Cells outlined in thick black lines in the matrix indicate comparison of burrows of the same architecture. 001, 007, and 0010 are subvertical burrows. 002, 004, 006, 008, and 009 are vertical burrows. 005 is a sinuous burrow. 003 is a U-shaped burrow. 0011-13 are J-shaped burrows. AG2 and AG8 are sinuous burrows. AG1, AG3, AG4, and AG6 are helical burrows. AG7 and AG5 are U-shaped burrows. Colors inside the matrix indicate the level of similarity: blue cells indicate highly similar burrows; orange cells indicate moderately similar burrows; red cells indicate dissimilar burrows. 


\section{APPENDIX 14.}

Scatter plots of data used in Spearman's rank correlation tests of Narceus americanus burrows. The x-axis is the relative clay and moisture content in the sediment. The y-axis is the measured value of the burrow property. Rs values indicate no significant relationship between sediment clay and moisture content to any of the eight different quantitative burrow properties tested.

\section{Narceus americanus}

\section{Clay Composition Sediment Moisture}

\section{Complexity}
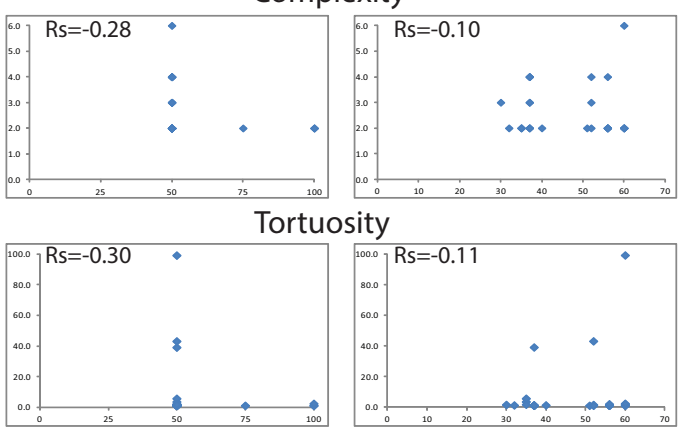

Average Circumference

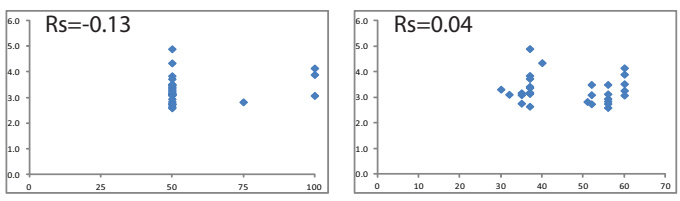

Total Length

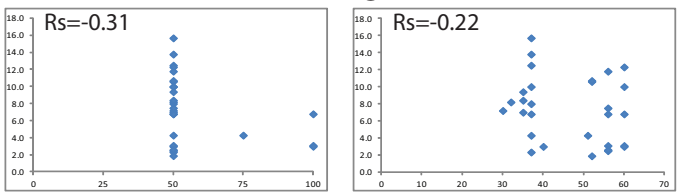

Average Width

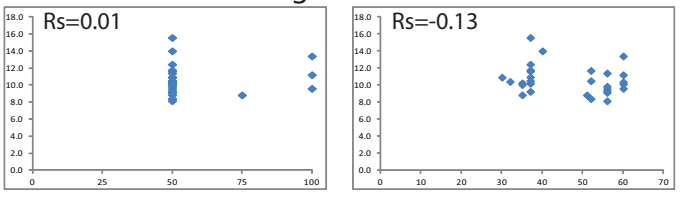

Average Height

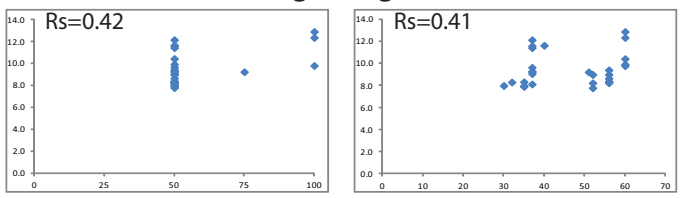

Maximum Depth

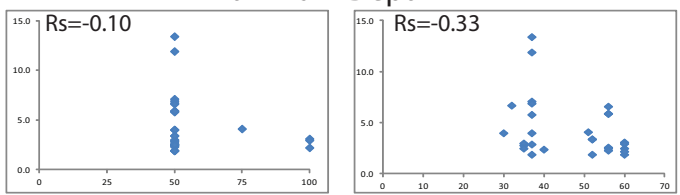

Average Slope

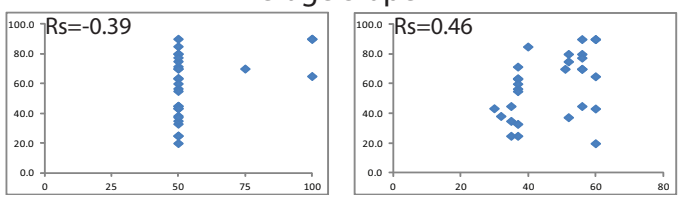




\section{APPENDIX 15.}

Scatter plots of data used in Spearman's rank correlation tests of Floridobolus penneri burrows. The x-axis is the relative clay and moisture content in the sediment. The $y$-axis is the measured value of the burrow property. Rs values indicate no significant relationship between sediment clay and moisture content to any of the eight different quantitative burrow properties tested.

Floridobolus penneri

Clay Composition Sediment Moisture

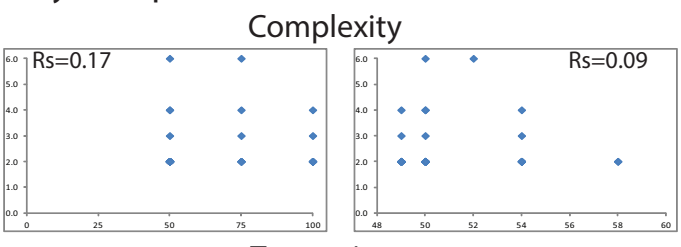

Tortuosity
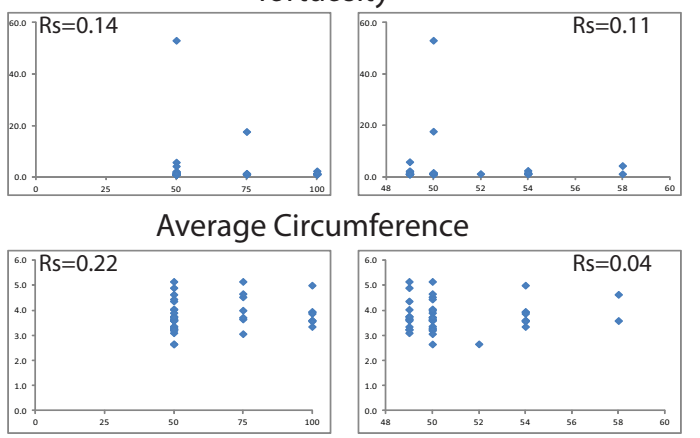

Total Length

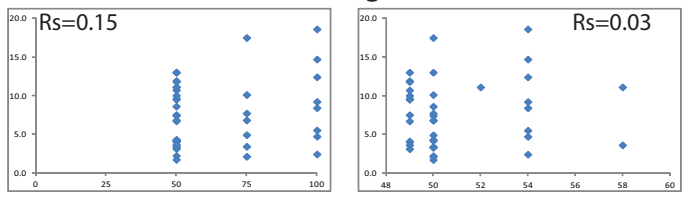

Average Width

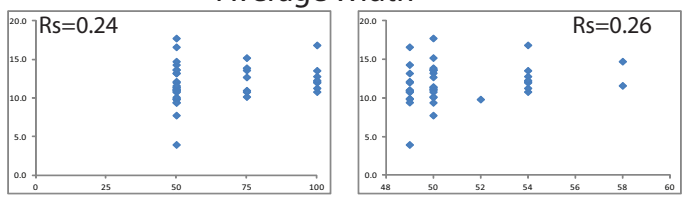

Average Height

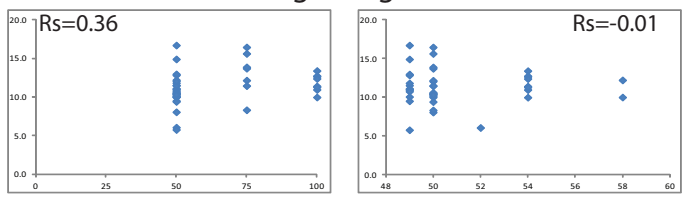

Maximum Depth

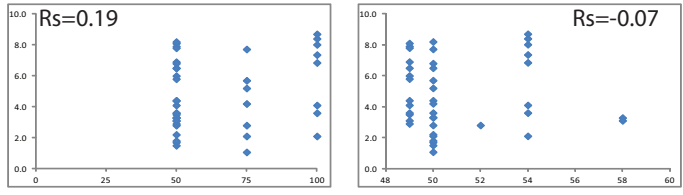

Average Slope

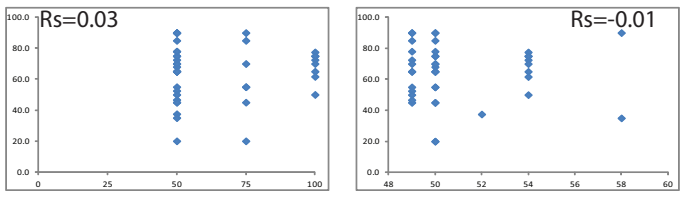

